GA-A15830

\title{
CONCRETE CONCENTRATOR PANEL DEVELOPMENT PROGRAM FOR SOLAROIL PROJECT, PHASE I
}

by

V. NICOLAYEFF, G. S. CHOW, and M. KOPLOY

\author{
Prepared under \\ Contract DE-AC03-79CS30308 \\ for the San Francisco Operations Office \\ Department of Energy
}

DATE PUBLISHED: MAY 1980

\section{GENERAL ATOMIC COMPANY}




\section{DISCLAIMER}

This report was prepared as an account of work sponsored by an agency of the United States Government. Neither the United States Government nor any agency Thereof, nor any of their employees, makes any warranty, express or implied, or assumes any legal liability or responsibility for the accuracy, completeness, or usefulness of any information, apparatus, product, or process disclosed, or represents that its use would not infringe privately owned rights. Reference herein to any specific commercial product, process, or service by trade name, trademark, manufacturer, or otherwise does not necessarily constitute or imply its endorsement, recommendation, or favoring by the United States Government or any agency thereof. The views and opinions of authors expressed herein do not necessarily state or reflect those of the United States Government or any agency thereof. 


\section{DISCLAIMER}

Portions of this document may be illegible in electronic image products. Images are produced from the best available original document. 


\section{NOTICE}

This report was prepared as an account of work sponsored by an agency of the United States Government. Neither the United States nor any agency thereof, nor any of their employees, makes any warranty, expressed or implied, or assumes any legal liability or responsibility for any third party's use or the results of such use of any information, apparatus, product or process disclosed in this report, or represents that its use by such third party would not infringe privately owned rights. 
GA-A15830

This book was prepared as an account of work sponsored by an agency of the United States Government. Neither the United States Government nor any agency thereot, nor any of their employees, makes any product or process disclosed of represents that its use mould not infringe pivid

commercial product, process, or sevvice by trade name, trademark, manufacturer, of otherwise, does

ot necessarily constivita of imply its endorsement, recommendation, or favoring by the United States Government or any agency thereol. The viows and opinions of authors expressed herein do not

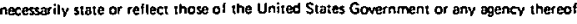

\title{
CONCRETE CONCENTRATOR PANEL DEVELOPMENT PROGRAM FOR SOLAROIL PROJECT, PHASE I
}

\author{
by \\ V. NICOLAYEFF, G. S. CHOW, and M. KOPLOY
}

Prepared under

Contract DE-AC03-79CS30308 for the San Francisco Operations Office

Department of Energy

GENERAL ATOMIC PROJECT 3315

DATE PUBLISHED: MAY 1980

\section{GENERAL ATOMIC COMPANY}




\section{THIS PAGE}

\section{WAS INTENTIONALLY \\ LEFT BLANK}




\section{THIS PAGE}

WAS INTENTIONALLY

LEFT BLANK 
CONTENTS

ABSTRACT . . . . . . . . . . . . . . . . . . . . . .

1. TNTRODUCTION . . . . . . . . . . . . . . . . . . . 1

2. MATERIAL SELECTION AND PANEL DESCRIPTION . . . . . . . . . . 4

2.1. Material Selection . . . . . . . . . . . . . . 4

2.2. Panel Description ................. 5

3. PANEL STRUCTURAL ANALYSIS . . . . . . . . . . . . . . . 11

3,1. Loading Conditions . . . . . . . . . . . . . . . 11

3.2. Structural Analysis . . . . . . . . . . . . . 13

4. PANEL DEVElOPMENT PROGRAM . . . . . . . . . . . . . . . . 19

4.1. Objectives . . . . . . . . . . . . . . . . 19

4.2. Test Program . . . . . . . . . . . . . . . 19

4.3. Test Procedure and Results . . . . . . . . . . . 21

4.3.1. Coating Tests .............. 21

4.3.2. Steel Form M1 . . . . . . . . . . . . . 32

4.3.3. Concrete Form $M 2$. . . . . . . . . . 36

4.3.4. Fiberglass-Coated Concrete Form M3 ....... . 40

4.3.5. Concrete Panels ................ . 42

4.3.6. Properties of Concrete... . . . . . . . 45

4.3.7. Measurement of slat Angles............. 47

4.3.8. Panel Supports and Installation . . . . . . . 55

4.4.' Conclusions and Recommendations . . . . . . . . . 55

4.4.1. Conclusions .............. . . 55

4.4.2. Recommendations for Fabrication of Panels

4.4.3. Recommendations for Phase II Tests....... . 59

5. ACKNOWLEDGMENTS . . . . . . . . . . . . . . . . . 61

6. REFERENCES ....................... . . 62

APPENDIX A: Slat Angle Measurement Data . . . . . . . . . . A-1

APPENDIX B: Comparison of Slat Angles of Panels and Forms . . . . A-2 
1. Typical collector row . . . . . . . . . . . . . . 2

2. Concentrator panel . . . . . . . . . . . . . . 7

3. Key dimensions of concentrator panel . . . . . . . . . . 8

4. Finite element mesh of panel . . . . . . . . . . . . . 12

5. Rotation at top edge of panel due to dead load vs position along panel length . . . . . . . . . . . . . . 15

6. Rotation near top edge of panel due to dead load vs position along panel length . . . . . . . . . . . . . . . . 16

7. Rocation at bottom edge of panel due to dead lnad va prisitinn Along panel length .................. 17

8. Rotation due to dead load vs panel aperture angle . . . . . . 18

9. Test panels and forms . . . . . . . . . . . . . . . 20

10. Steel form $\mathrm{Ml}$. . . . . . . . . . . . . . . . 33

11. Details of steel form M1 . . . . . . . . . . . . . . 34

12. Fabrication of concrete form M2 . . . . . . . . . . . . 37

13. Outline of concrete forms . . . . . . . . . . . . . 39

14. Fabrication of fiberglass-coated concrete form M3 . . . . . . 41

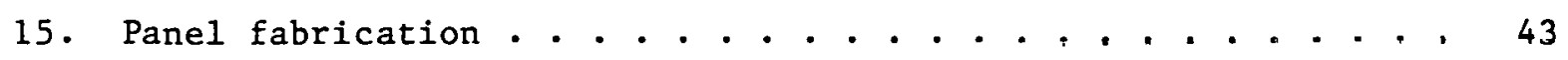

16. Pancls in place... . . . . . . . . . . . . . . . 46

17. Measurement of angle $\phi$ with laser inspection fixture . . . . 48

18. Measurement with laser inspection fixture . . . . . . . . . 49

19. Key to readings taken with laser inspection fixture ...... 53

\section{TABLES}

1. Plerlal speclfications for concentrator panels . . . . . . 6

2. Dimensions controlling panel geometry . . . . . . . . . . . 9

3. Results of structural analysis . . . . . . . . . . . . . 14

4. Concentrator panel development program schedule . . . . . . . 22

5. Key program dates . . . . . . . . . . . . . . . . 23

6. Properties of concrete . . . . . . . . . . . . . . . 24

7. Summary of laser inspections . . . . . . . . . . . . . . 29

8. Difference between achieved and desired slat angles . . . . . 30

9. Comparison of slat angles on panels and forms . . . . . . . . . 31 


\section{INTRODUCTION}

In the General Atomic (GA) solar steam supply system proposed for the solar thermal enhanced oil recovery (SolarOil) project, the energy from the sun is collected by a primary concentrator and focused onto a receiver. The fluid in the receiver is heated to approximately $320^{\circ} \mathrm{C}\left(608^{\circ} \mathrm{F}\right)$ and is used to produce saturated steam at $4.8 \mathrm{MPa}(700 \mathrm{psi}), 262^{\circ} \mathrm{C}\left(503^{\circ} \mathrm{F}\right)$, and $80 \%$ quality. The plant output is equivalent to that of a $2.4 \mathrm{MW}\left(8.33 \times 10^{6} \mathrm{Btu} / \mathrm{h}\right)$ oilfired steam generator.

The collector field consists of 50 rows of fixed-mirror solar concentrators (FMSCs) developed by GA. The rows are $175.6 \mathrm{~m}$ (576 ft) long and are spaced at 7.62-m (25-ft) intervals (Fjg. 1). The field has $31,600 \mathrm{~m}^{2}$ $\left(340,800 \mathrm{ft}^{2}\right)$ of mirror surface and occupies approximately $66,000 \mathrm{~m}^{2}$ (16 acres) of 1and. Each of the 1600 FMSC concrete panels is $5.5 \mathrm{~m}$ (18 ft) long with a $3.6 \mathrm{~m}$ (11 ft $10 \mathrm{in.)}$ aperture. The panel has 71 facets on its interior face which are covered with mirror strips. The facets are located with their centers on the periphery of a so-called focal circle and are oriented such that they focus the sun's rays onto a line which is also located on the periphery of the circle. As the sun moves in the north-south plane, the focus line of the reflected rays moves around the periphery of the focal circle. A lightweight receiver follows the focus line and captures the reflected insolation (Fig. 1).

For optimum concentrator performance, all the reflected rays must hit an 89-mm (3.5-in.) wide receiver aperture located up to $4.78 \mathrm{~m}(15.70 \mathrm{ft})$ away. This requires that when the following sources of errors are summed up, the mirror pointing accuracy is within +0.25 deg of its predetermined angle:

1. Form imprecision.

2. Concrete shr1nkage and curing distortions.

3. Nonuniformities in glass and adhesive thickness. 

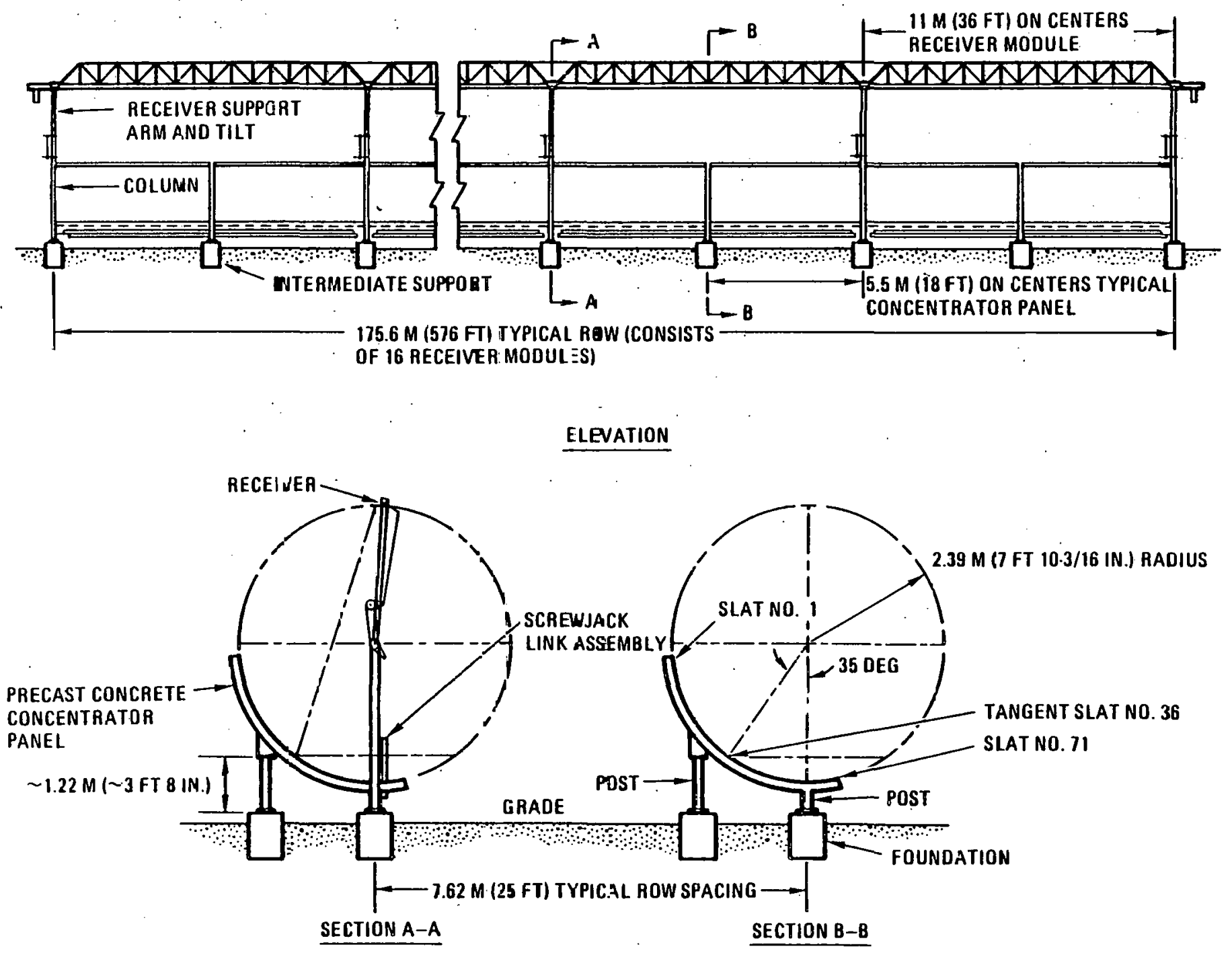

Fig. 1'. Typical collector row 
4. Sag and twist of the concentrator panel.

5. Misalignment of the concentrator panel and settlement of supports.

Achievement of such a close tolerance with concrete or any other material has been believed difficult, especially since the cost of the panels must be comparable to that of architectural concrete to keep the plant cost competitive. Accordingly, a good portion of the panel program during the Solaroil phase I effort was dedicated to (1) optimizing a panel configuration using analytical studies and (2) testing panels with the selected configuration. Determination of the achievable accuracy of the slat angles was emphasized along with the development of economical methods of manufacturing the panels and forms.

This report covers concentrator panel work conducted between October 1 , 1979 and April 30, 1980 and supplements the information given in Ref. 1 . 


\section{MATERIAL SELECTION AND PANEL DESCRIPTION}

\subsection{MATERIAL SELECTION}

Reinforced concrete was selected for the panels, and the panels were designed for performance, ease of construction, and economy. The advantages of reinforced concrete are its

1. Relatively low cost.

2. Thermal compatibility with mirror glass.

3. Good durability under a wide variety of exposures.

4. Good strength and hardness.

5. Relatively low volume shanges.

6. Slow response to temperature change.

7. Low maintenance requirements,

8. Ability to be rapldly cast intu a wlde variety of shapes and forms. using locally available materials and unskilled local labor.

Steel and plastic were also considered. Steel was rejected because of its high cost, the long lead time required for delivery, and difficulties with achieving proper tolerances. Plastic was excluded because of its uncertain durability under exposure, although it is still being considered for future projects because of its low unit weight. 
Table 1 gives the material specifications for the concentrator panels. These properties could be modified for the final design of the panels.

\subsection{PANEL DESCRIPTION}

Figure 2 shows the concentrator panel. It is essentially a thin, reinforced concrete shell which can be fabricated at a precasting yard or a construction site, depending upon labor availability and economics. It is designed to maintain slat angle accuracy to within $\pm 0.25 \mathrm{deg}$ and to remain structurally sound during fabrication, handling, and its 15-yr life in the field.

The initial slat angle accuracy is achieved by casting the panel on a precision-made form using a properly designed concrete mixture and by extensively vibrating the concrete. The panels are steam-cured overnight at $49^{\circ} \mathrm{C}$ $\left(120^{\circ} \mathrm{F}\right)$. The following day they are strong enough to be stripped from the forms. Form slats have a 0.5-to 1.0-deg draft for easier stripping. The draft'makes the width of all the slats relatively constant. After stripping, the panels are washed to remove grit and are then sealed to minimize dusting and to provide a good bonding surface for the mirrors.

The back of the panel is equipped with inserts for lifting devices and bolts which attach the panel to supports. Leveling nuts are provided for initial alignment of the panel and to compensate for excessive settlement of supports.

Figure 3 gives the key dimensions and tolerances of the reference concentrator panel. Table 2 gives the dimensions that control the panel geometry.

At first, 5.5-m (18-ft) and $11.0-\mathrm{m}(36-\mathrm{ft})$ long panels with $3.6 \mathrm{~m}$ (11.ft 10 in.) apertures were considered. Panels of both lengths were analyzed by the finite element method. Section 3 gives the details of the structural analysis. The preliminary results indicated that the 5.5-m (18-ft) long 
TABLE 1

MATERIAL SPECIFICATIONS FOR CONCENTRATOR PANELS

\section{Properties}

Specified compressive strength at age of 28 days $=31.0 \mathrm{MPa}$ (4500 psi)

Specified compressive strength at time of stripping $=20.7 \mathrm{MPa}$

(3000 psi)

Flexural strength at age of 28 days $=3.45 \mathrm{MPa}$ (500 psi)

Elastic modulus $=17.2 \mathrm{GPa}\left(2.5 \times 10^{6} \mathrm{psi}\right)$

Linear coefficient of thermal expansion $=5.6 \times 10^{-6} /{ }^{\circ} \mathrm{C}\left(3.1 \times 10^{-6} /{ }^{\circ} \mathrm{F}\right)$

Unit weight $=2.32 \mathrm{Mg} / \mathrm{m}^{3}\left(145 \mathrm{lb} / \mathrm{ft}^{3}\right)$

Maximum drying shrinkage $=600 \times 10^{-6} \mathrm{~m} / \mathrm{m}$ (in./in.)

Long-term creep plus elastic deformation $=2.5$ times elastic deformation (maximum)

Materials and mix proportions for panel concrete

Portland cement $=$ ASTM C 150, type I, II, or III

Aggregate $=$ ASTM C 33 [maximum size $=9.5 \mathrm{~mm}$ ( $3 / 8$ in.)]

Admixtures $=$ as selected by manufacturer

Mix proportions - as selected by malufacturer

Slump $=90 \mathrm{~mm}(3.5 \mathrm{in.})$

Reinforcement

Bars $=$ ASTM A 615, grade 60

Welded wire fabric $=$ ASTM A 185

Steel fibers = as selected by manufacturer

Miscellaneous

Specified compressive strength of footing concrete at age of 28 days $=$ 20.7 МРя (30กก psi)

Form coatings, parting compounds, inserts, and brackets $=$ as selected by manufacturer

Support posts and bearing plates = ASTM A 36

Anchor bolts $=$ ASTM A 325 


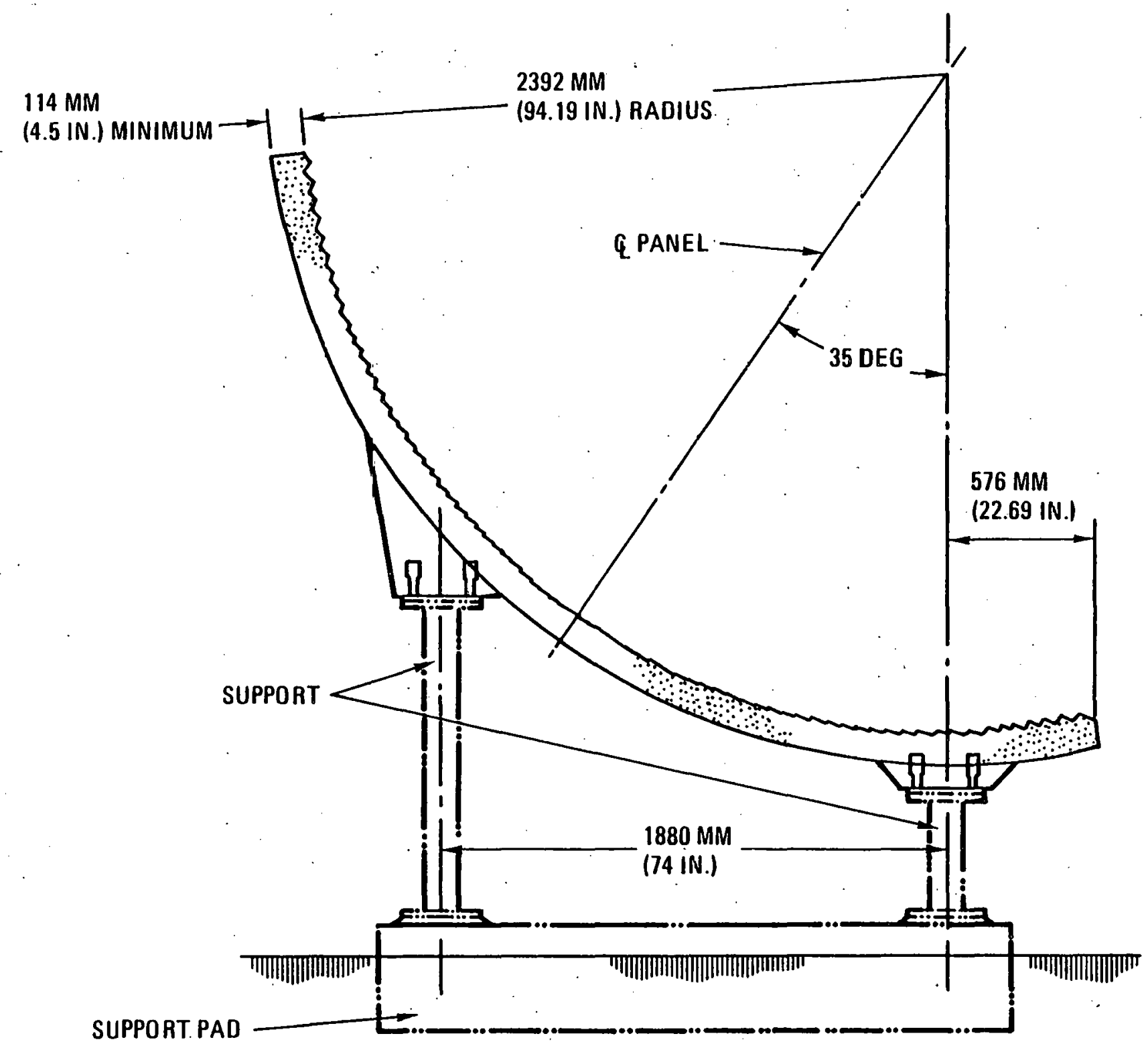

Fig. 2. Concentrator panel 

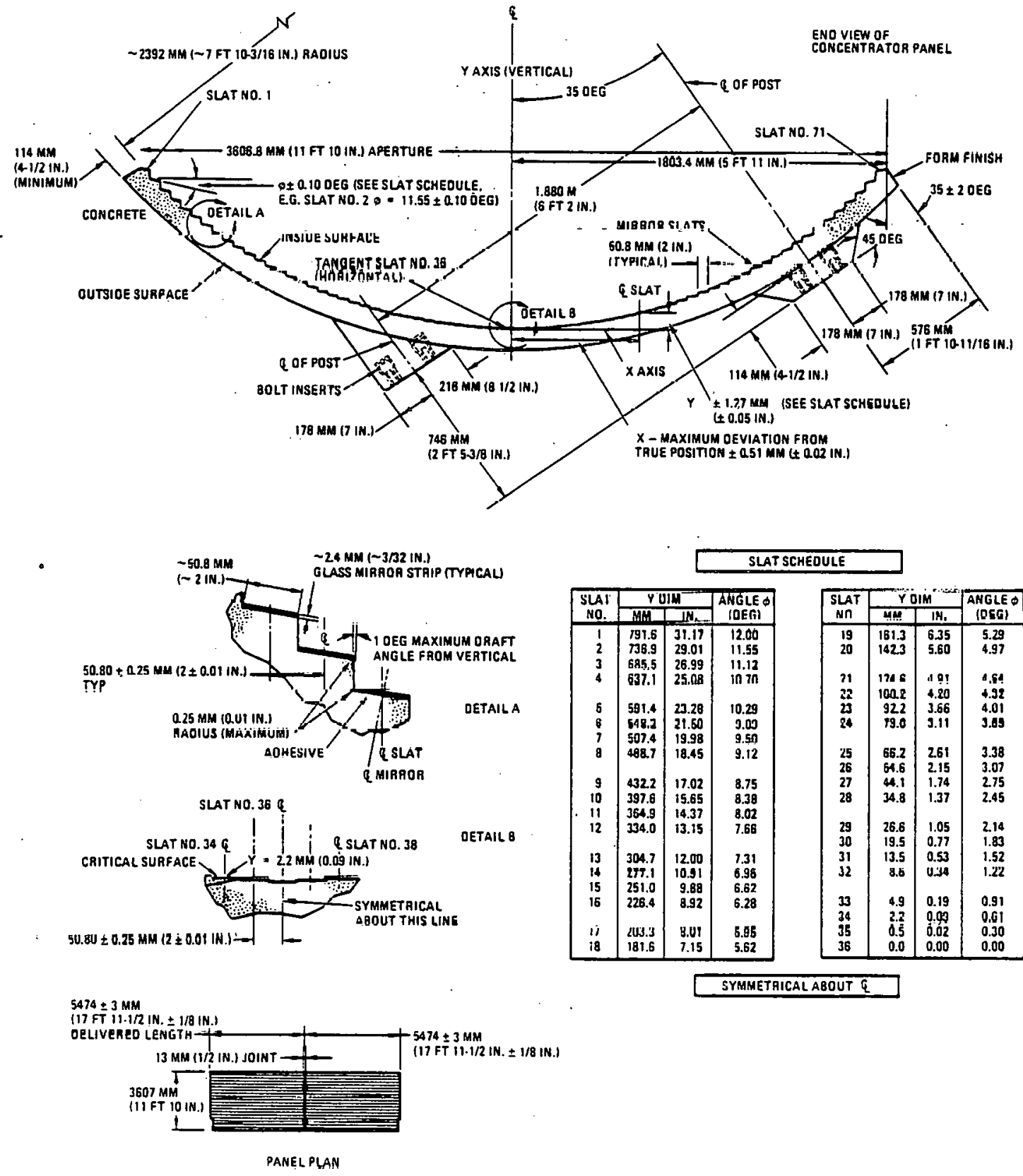

Fig. 3. Key dimensions of concentrator panel 
Panel aperture (chord between opposite mirror edges)

Panel aperture angle

Focal radius (derived value)

Mirror width (nominal)

Mirror thickness

Hirror adhesive thickness (maximum)

Number of mirror facets

Panel length (nominal)

Panel thickness (minimum)

Panel support intervals (ón centers)

Panel height from ground

Panel tilt angle

Vertical height adjustment of panel support post

Lateral adjustment of panel support post

Height tolerance of panel foundation (top of concrete) relative to design elevation

Panel slope along longitudinal axis
$3.61 \mathrm{~m}(142 \mathrm{in.})$

$96 \mathrm{deg}$

$2.39 \mathrm{~m}$ ( 94.19 in.)

$50.8 \mathrm{~mm}$ ( 2 in.)

$2.3 \mathrm{~mm}(0.090 \mathrm{in.})$

$0.13 \mathrm{~mm}(0.005$ in.)

71

$5.49 \mathrm{~m}(18 \mathrm{ft})$

$114 \mathrm{~mm}$ (4.5 in.)

5.49 แ (10 Ft)

Sufficient to ensure clearance for drive train components

$35 \mathrm{deg}$

$\pm 19.0 \mathrm{~mm}( \pm 0.75 \mathrm{in}$.

$\pm 6.4 \mathrm{~mm}$ (+0.25 in.)

$\pm 12.7 \mathrm{~mm}(+0.5 \mathrm{in.})$

2 in 1000 minimum, 25 in 1000 maximum 
panels were structurally adequate without prestressing and the short- and long-term deflections acceptable. The $11.0-\mathrm{m}(36-\mathrm{ft})$ long panels required prestressing, an intermediate support, or a stiffening beam to control deflections. Cursory analysis of a $1.83-\mathrm{m}(6-\mathrm{ft})$ long panel indicated that its stresses and deflections were smaller than those for the 5.5-m (18-ft) long panels.

From a handing and installation point of view, the $11.0-\mathrm{m}$ (36-ft) long panels could create handling and shipping problems, and the $1.83-\mathrm{m}$ (6-ft) long panels would require too much handing and too many support posts. For these reasons, the $5.5-\mathrm{m}(18-\mathrm{ft})$ long panels were selected as a standard. 


\section{PANEL STRUCTURAL ANALYSIS}

The 5.5-m. (18-ft) and 11.0-m (36-ft) long panels were analyzed by the finite element method using the MODSAP computer program (Ref. 2). The panel model consisted of 72 plate shell elements (Fig. 4). The panel was assumed to be fixed at the four points where the supports are located and was oriented as shown in Fig. 2 .

\subsection{LOADINF COCNDTTTONS}

The loading conditfons included dead load, live load, wind load, and seismic load. The live loads investigated were

1. A $290-\mathrm{N} / \mathrm{m}(20-1 \mathrm{~b} / \mathrm{ft})$ load on top of the panel to account for the weight of the receiver assembly when it is positioned on top of the panel.

2. A 4.4-kN (1000-1b) moving load, which represents the weight of the machine that installs mirrors on the slats and the other loads expected during maintenance of the panels.

3. A rain load equivalent to a $65-\mathrm{mm}$ (2.55-in.) deep puddle in the water-retaining portion of the panel (see Fig. 2).

4. An ice load equivalent to a 50-mm (2-in.) layer of water over the entire panel surface.

The wind load was calculated assuming a $145-\mathrm{km} / \mathrm{h}(90-\mathrm{mi} / \mathrm{h})$ gust. The seismic loads were calculated using a static vertical acceleration of $0.19 \mathrm{~g}$ and a static lateral acceleration of $0.28 \mathrm{~g}$. 


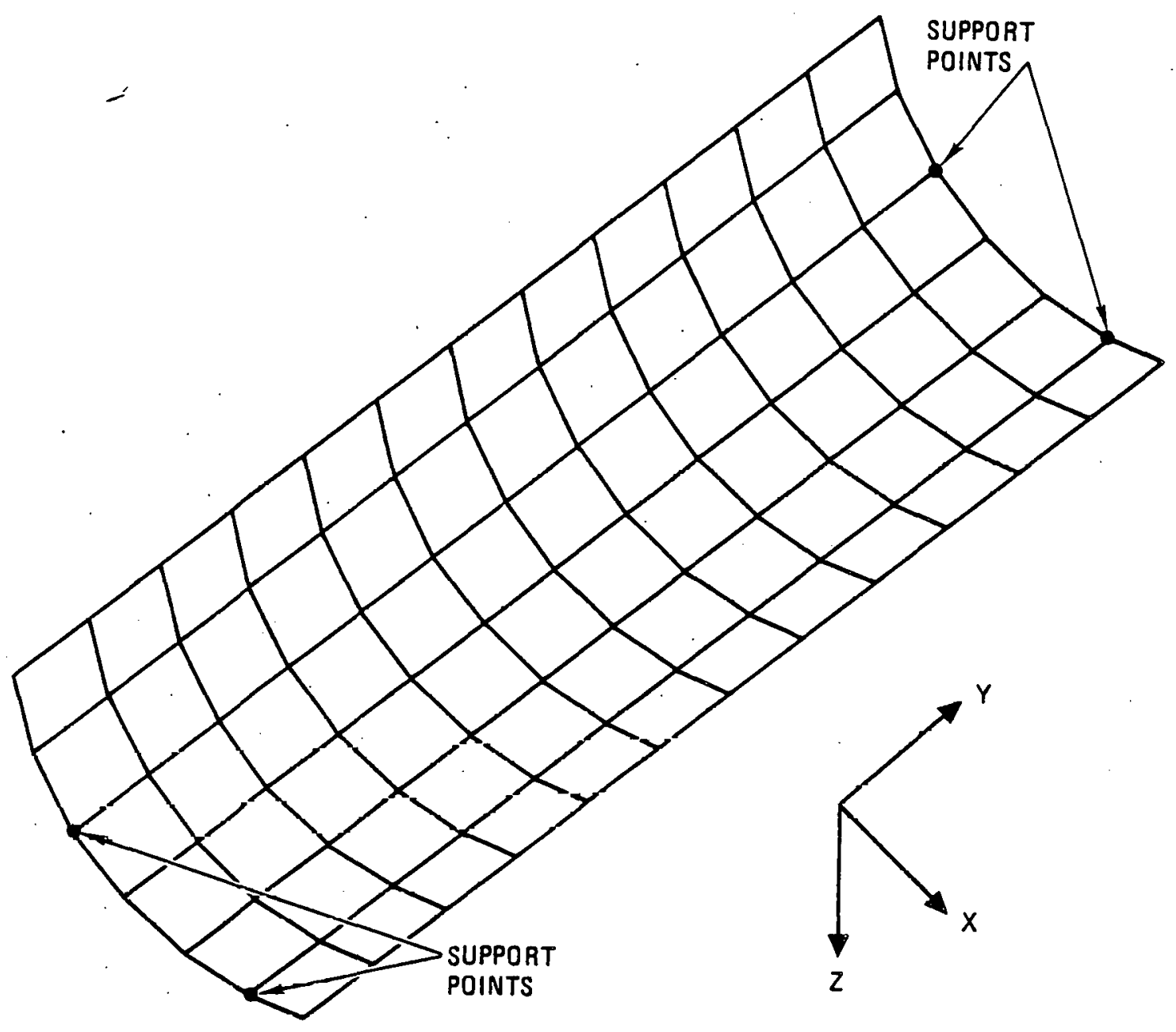

Fig. 4. Finite element mesh of panel 


\subsection{STRUCTURAL ANALYSIS}

Table 3 presents the results of the structural analysis. The stresses produced by the loads described above were low for panels of both lengths. Long-term displacements and rotations due to dead load were acceptable for the 5.5-m (18-ft) panel but too large for the 11.0-m (36-ft) panel. The results clearly indicated that panel design is controlled by displacement rather than stress.

The $1.83-m(6-f t)$ long panel was analyzed for dead load only. The stresses and displacements for this panel were lower than those for the 5.5-m (18-ft) long panel, as expected, and they are anticipated to be lower for the other loadings as woll.

Figures 5 through 7 show the rotations expected for the $1.83-\mathrm{m}(6-\mathrm{ft})$, $5.5-m(18-f t)$, and $11.0-m(36-f t)$ long panels due to dead load only. The rotations at the top edge of the panel, near the top, and at the bottom edge are plotted as a function of panel length. The $1.83-\mathrm{m}(6-\mathrm{ft})$ and $5.5-\mathrm{m}$ (18-ft) long panels have moderate rotations of similar magnitude; the 11.0-m (36-ft) panel has much larger rotations. This shows that from a performance point of view, the $11.0-\mathrm{m}(36-\mathrm{ft})$ long panel will not meet the maximum rotation criterion of $+0.25 \mathrm{deg}$ unless modifications are made to the panel design, such as increasing panel thickness, providing intermediate supports, adding stiffening beams, or prestressing the panel. These modifications will increase the cost of the panel.

Figure 8 shows the rotations of the $1.83-\mathrm{m}(6-f t)$ and the $5.5-\mathrm{m}(18-f t)$ long panels due only to dead load as a function of panel aperture angle. The rotations for both panels are well within the acceptable limits. Thus, it can be concluded that panel design is controlled by deflection rather than stress and, for lengths up to at least $5.5 \mathrm{~m}(18 \mathrm{ft})$, by aperture rather than length. 
TABLE 3

RESULTS OF STRUCTURAL ANALYSIS

\begin{tabular}{l|c|c}
\hline & $\begin{array}{c}5.5-\mathrm{m}(18-\mathrm{ft}) \\
\text { Long Panel }\end{array}$ & $\begin{array}{c}11.0-\mathrm{m}(36-\mathrm{ft}) \\
\text { Long Panel }\end{array}$ \\
\hline $\begin{array}{l}\text { Maximum tensile stress due to } \\
\text { loads described in Section 3.1. } \\
\text { [MPa (psi)] }\end{array}$ & $1.54(224)$ & $2.10(305)$ \\
$\begin{array}{l}\text { Maximum long-term vert1cal } \\
\text { displacement due to dead load } \\
\text { (elastic and creep) [mm (in.)] }\end{array}$ & $3.6(0.14)$ & $22.3(0.88)$ \\
$\begin{array}{l}\text { Maximum long-term rotation due } \\
\text { to dead load (elastic and } \\
\text { creep) (deg) }\end{array}$ & -0.13 & -0.49 \\
\hline
\end{tabular}

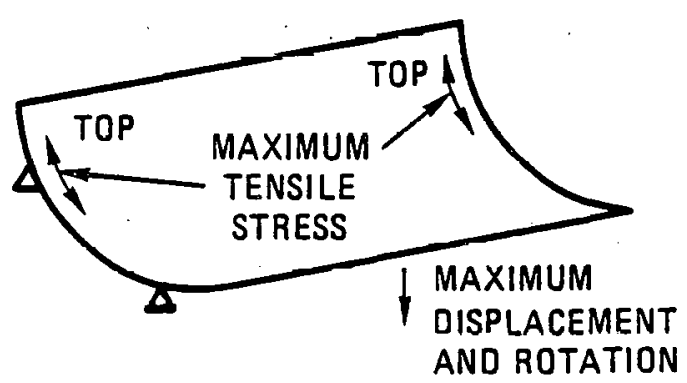

5.5-M (18-FT) PANEL

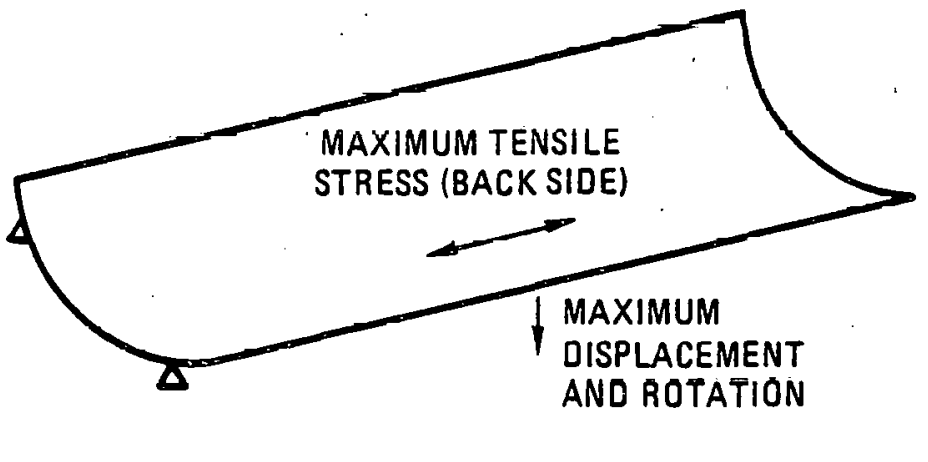

11.0-M (36-FT) PANEL 


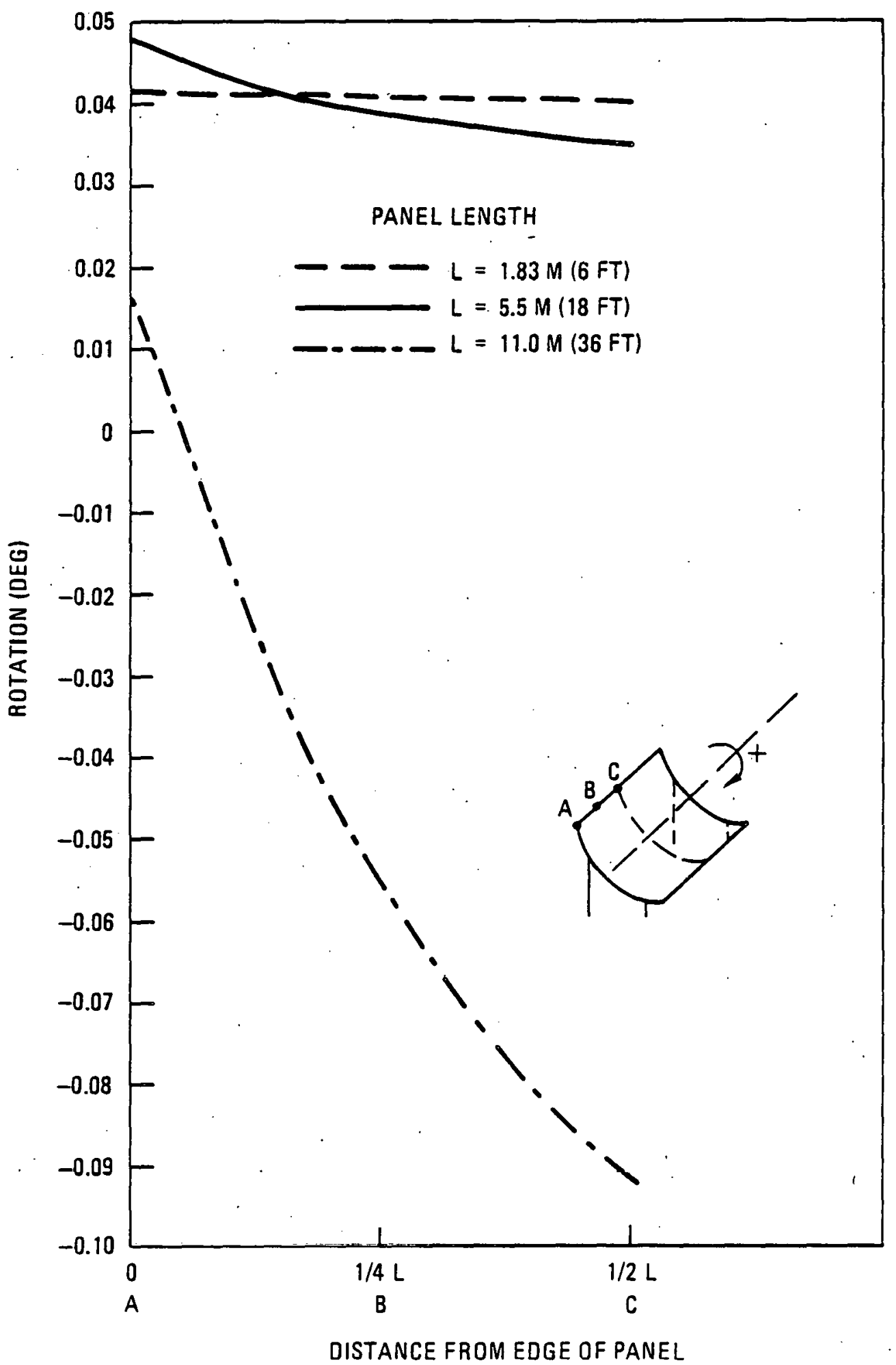

Fig. 5. Rotation at top edge of panel due to dead load vs position along panel length 


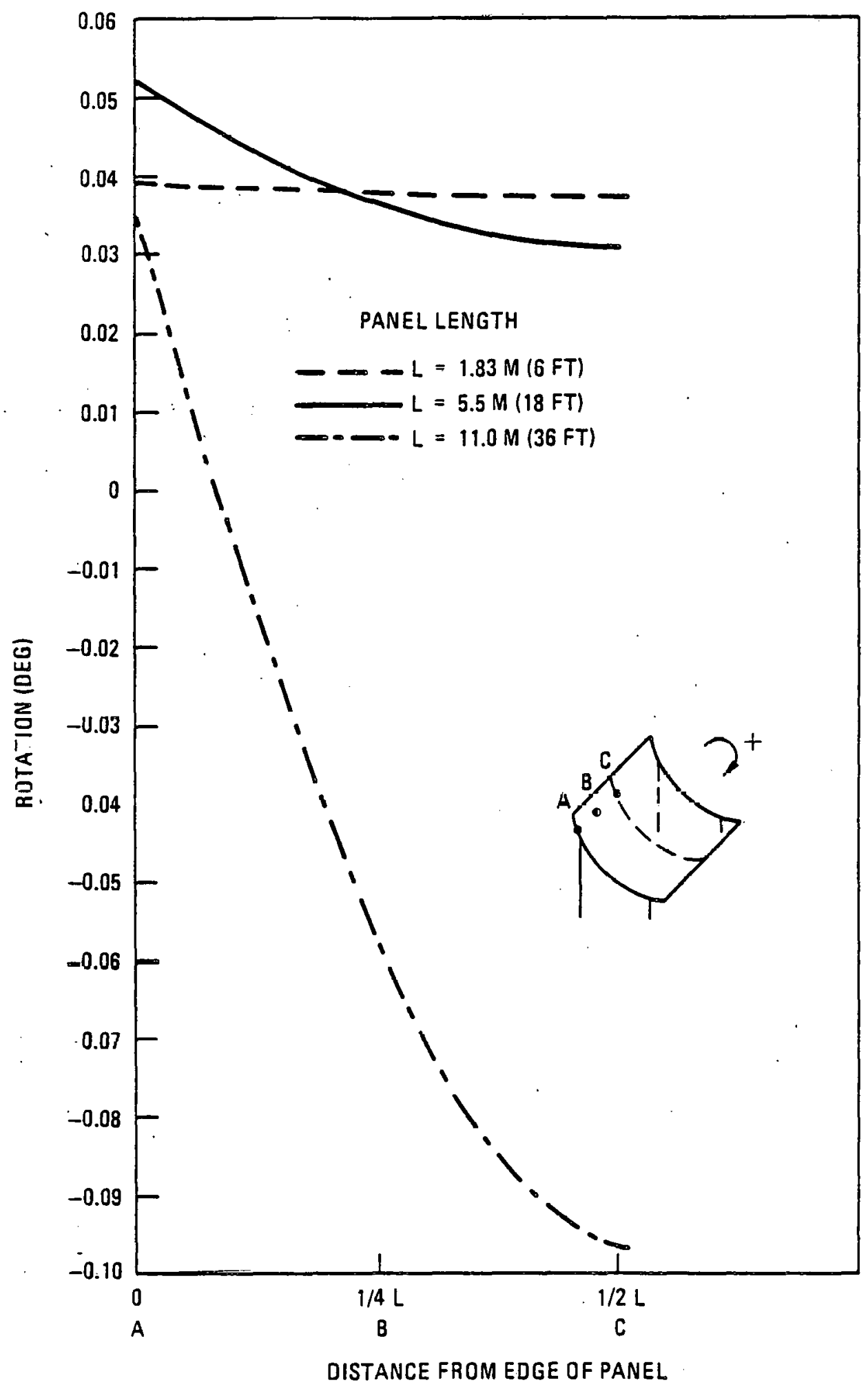

Fig. 6. Rotation near top edge of panel due to dead load vs position along panel length 


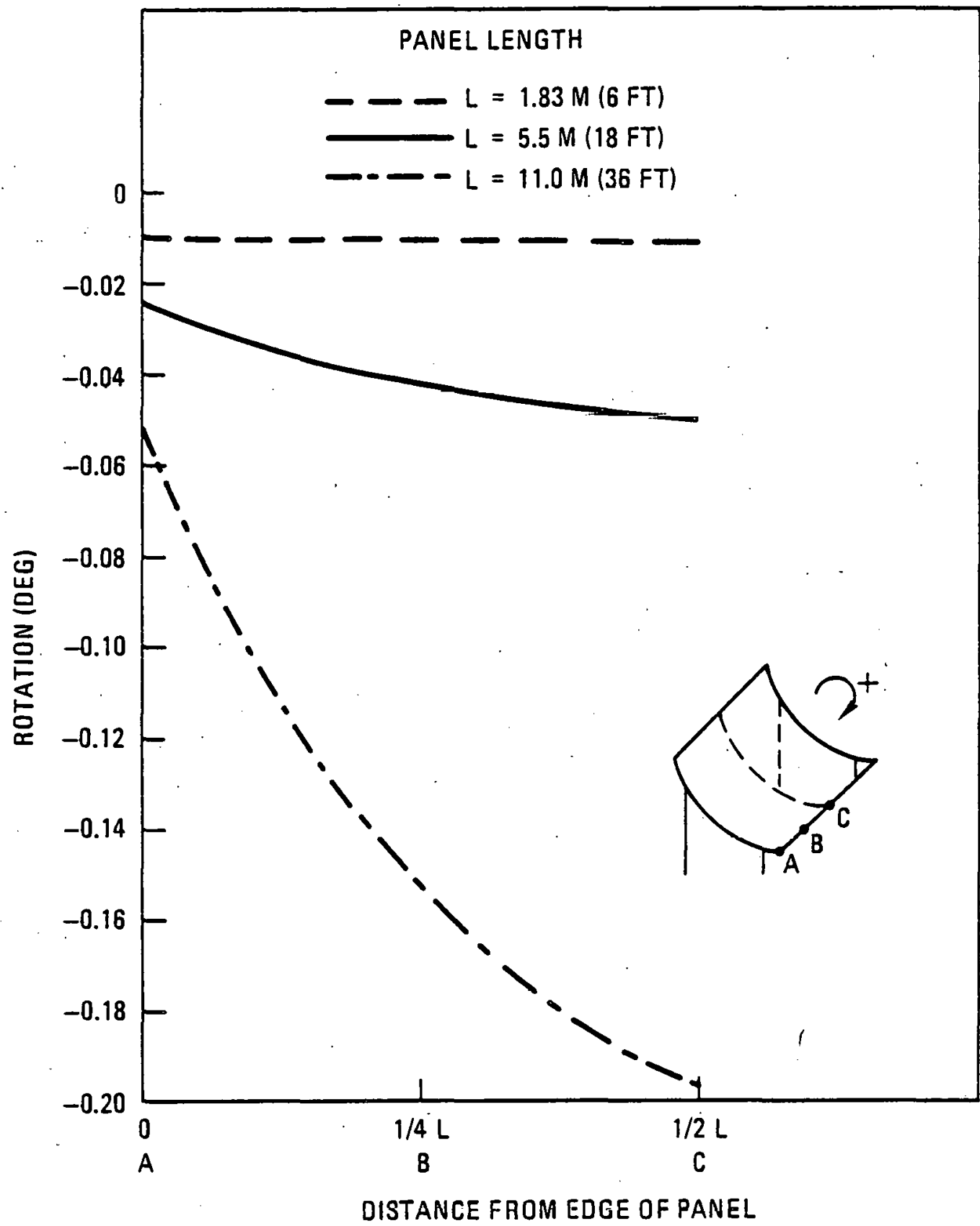

Fig. 7. Rotation at bottom edge of panel due to dead load vs position along panel length 


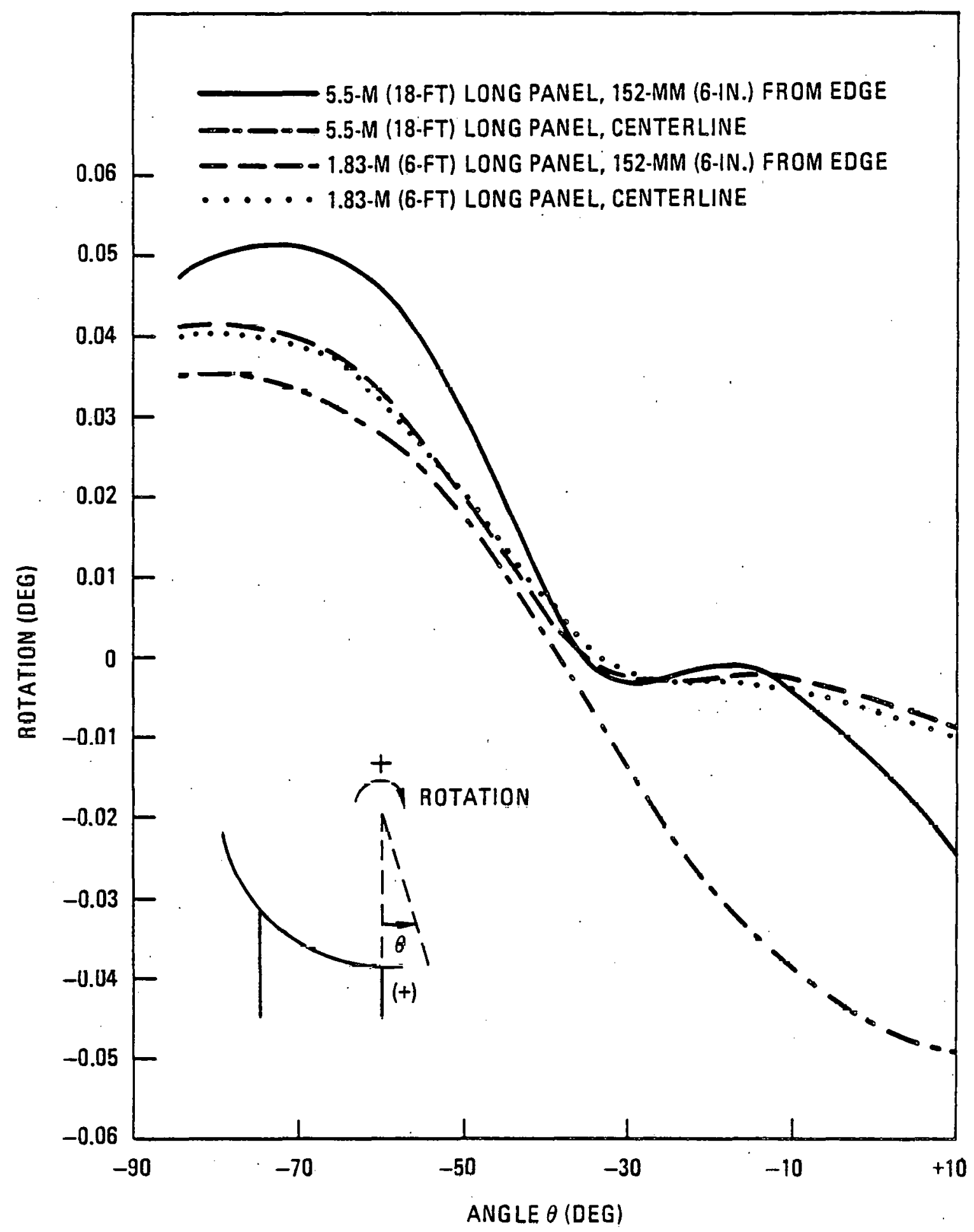

Fig. 8. Rotation due to dead Load vs panel aperture angle 


\section{PANEL DEVELOPMENT PROGRAM}

\subsection{OBJECTIVES}

The unusually tight tolerances on the mirror slats, the uncertainties associated with the fabrication of the panels and forms, and the concern about the structural behavior of the panels necessitated a development program to give credibility to the GA design and to reduce risks. The objectives of the program are given below.

1. To determine the achievable slat angle accuracy of
a. Steel forms.
b. Concrete forms.
c. Precast concrete concentrator panels.

2. To investigate economical and expedient methods of mass producing panels and forms, including
a. Optimum panel configuration.
b. Pancl reinforcement and emhedments.
c. High-reuse, low-maintenance forms.
d. Rapid curing.
e. Rapid inspection.
f. Rapid installation and alignment.

\subsection{TEST PROGRAM}

The test program comprised coating tests, fabrication of forms and panels, and measurement of slat angles on forms and panels. Figure 9 depicts the products manufactured during the test program. They consisted of a precision-made steel form (M1), two concrete forms (M2 and M3), and three 


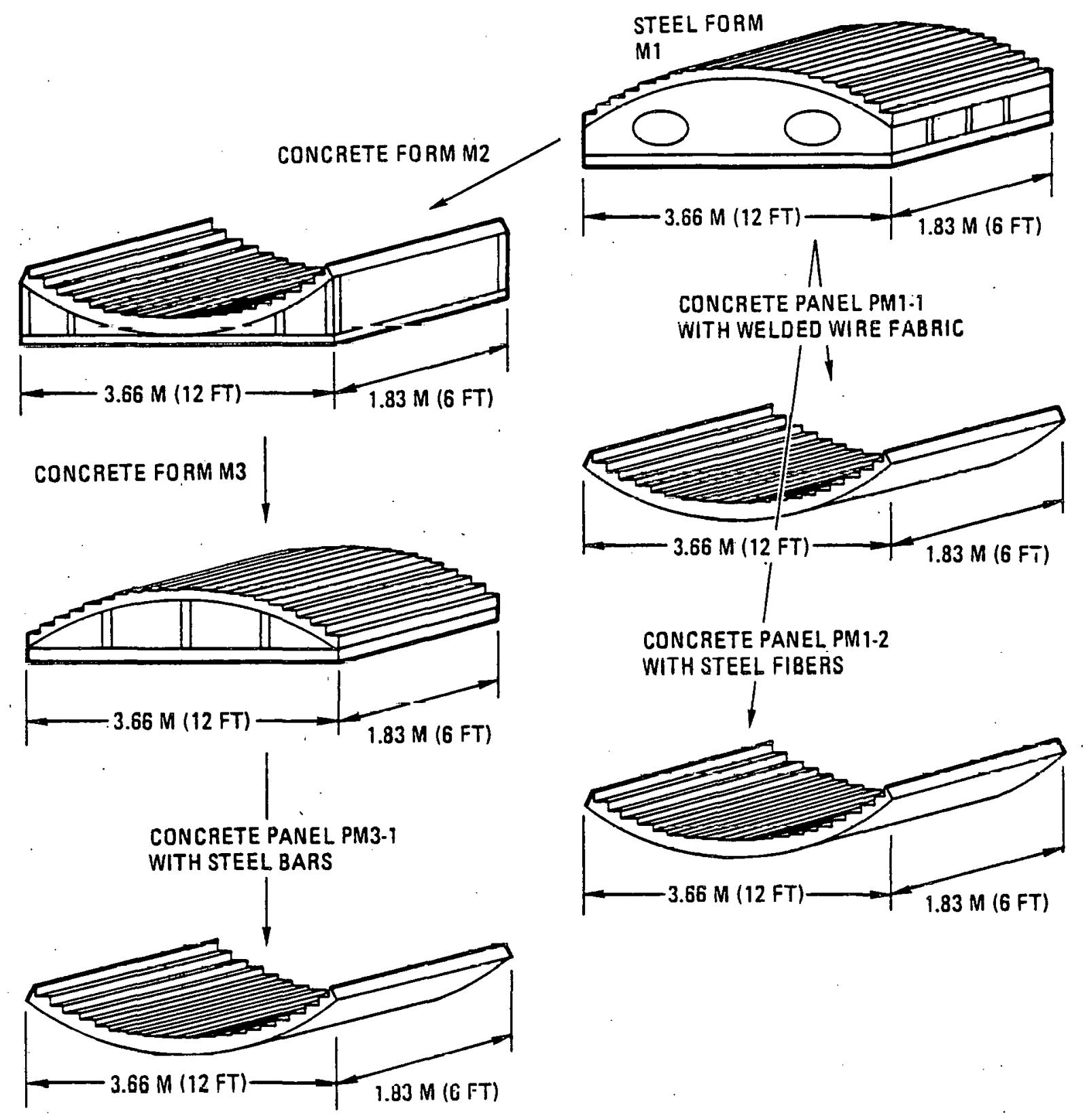

Fig. 9. Test panels and forms 
panels (PM1-1, PM1-2, and PM3-1). Concrete form M2 and panels PM1-1 and PM1-2 were cast from form M1; form M3 was cast from M2; and pane1 PM3-1 was cast from M3.

The length of all test panels and forms was $1.83 \mathrm{~m}(6 \mathrm{ft})$. The thickness and aperture of the test panels were the same as those of the production panels, i.e., $115 \mathrm{~mm}(4.5 \mathrm{in.})$ and $3.6 \mathrm{~m}$ (11 ft $10 \mathrm{in.}$ ), respectively. The shorter length was justified since the results of the panel analysis showed that panel design and performance are controlled by thickness and aperture for lengths up to $5.5 \mathrm{~m}$ (18 ft).

Table 4 presents the program schedule. This tight schedule allowed no slack to make up for lost time; hence, defects discovered during fabrication of forms and panels were not corrected if they did not affect the overall objectives of the program.

\subsection{TEST PROCEDURE AND RESULTS}

Table 5 shows the key dates of the program. Table 6 gives the properties of the concrete, Table 7 summarizes the laser inspections, and Tables 8 and 9 present the slat angle measurements.

\subsubsection{Coating Tests}

Coating tests were conducted on small 0.6 by $1.2 \mathrm{~m}$ ( 2 by $4 \mathrm{t} \tau$ ) panels. The purpose of these tests was to find suitable parting compounds, sealing compounds, and surface hardeners for fabrication of large concrete forms and panels: The concrete constituents, placement method, and steam curing conditions were the same as those which will be used for the large panels.

The parting compounds that had to be mixed with diesel fuel left the forms dirty and were considered inadequate. Most of the other commercially available parting compounds that were tested were fair to good. The material selected for casting the panels was Protex Form Release, which performed well 
CONCENTRATOR PANEL DEVELOPMENT PP.OGRAM SCHEDULE

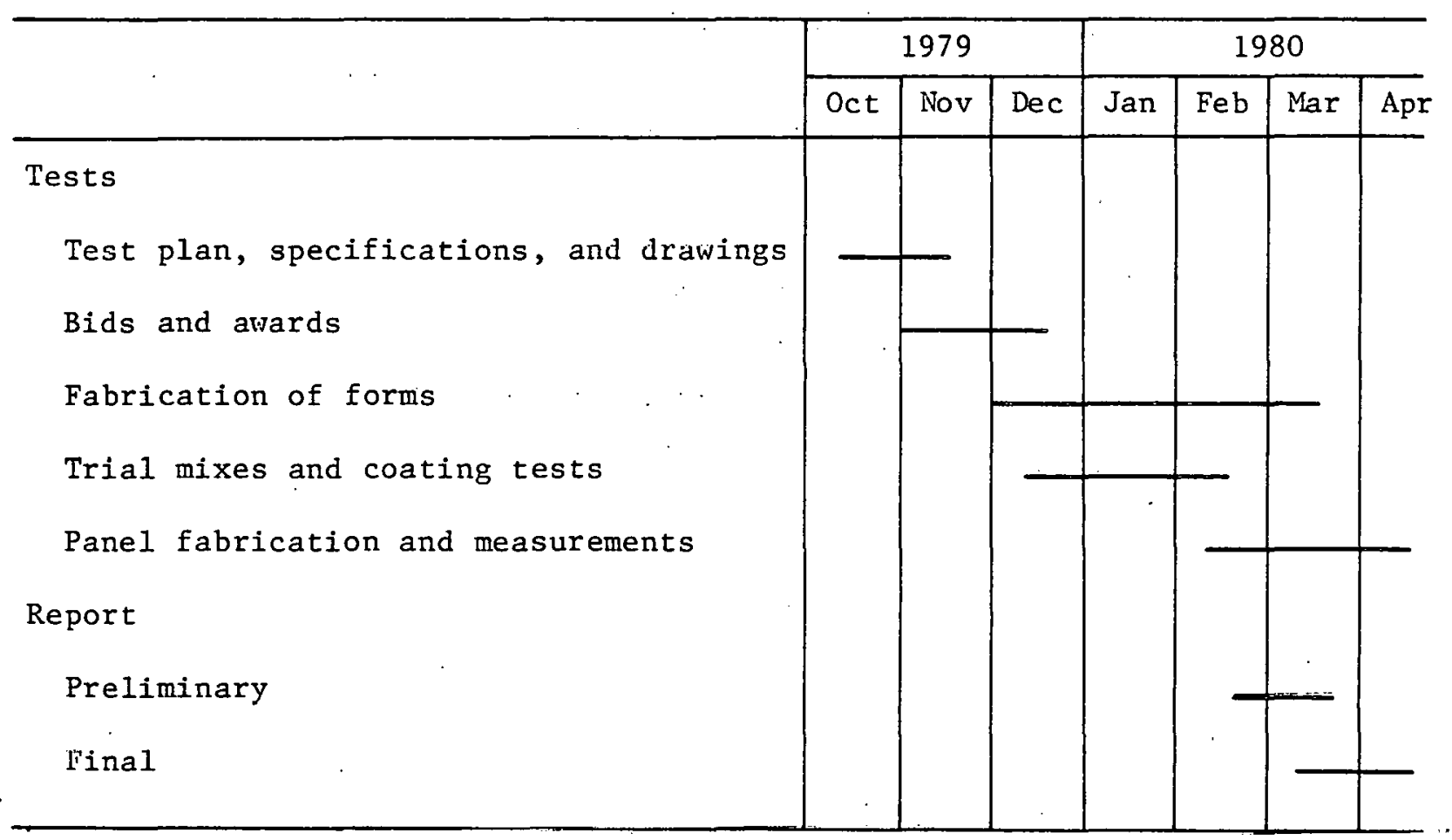


TABLE 5

KEY PROGRAM DATES

$11 / 8 / 79$

$12 / 11 / 79$

$12 / 28 / 79$

$1 / 24 / 80$

$2 / 8 / 80$

$2 / 18 / 80$

$2 / 27 / 80$

$3 / 3 / 80$

$3 / 4 / 8 n$

$3 / 7 / 80$

$3 / 8 / 80$

$3 / 11 / 80$

$3 / 12 / 80$

$3 / 13 / 80$

$3 / 14 / 80$

$3 / 17 / 80$

$3 / 18 / 80$

$3 / 18 / 80$

$3 / 19 / 80$

$3 / 24 / 80$

$4 / 15 / 80$

$4 / 30 / 80$
Request for quote sent out

Purchase order issued

First issue of shop drawings completed

Fabrication of laser inspection fixture completed

Laser inspection fixture delivered to panel manufacturer

M1 bulkheads and skid delivered to panel manufacturer

22 slats installed on $\mathrm{Ml}$

Assembly of $M 1$ completed

M1. slats measured with laser inspection fixture

M2 cast

M2 stripped and PMI-1 cast

Ge1 coat applied on M2 (for M3 surface) and PM1-1 stripped PM1-2 cast and fiberglass applied on $M 2$

PM1-2 stripped

M3 cast

M3 stripped

PM3-1 cast

PM1-1 and PM1-2 installed on supports

PM3-1 stripped

PM3-1 installed on supports

All required slat angle measurements completed

Shimming of $M 1$ completed 
TABLE 6

PROFERTIES OF CONCRETE (a)

A. MATERIALS

Portland cement: Mojave type I/II low alkali; meets ASTM C 150.

Mo jave type V, used only in trial mix Mo. 3; meets ASTM C 150.

Water: spigot water.

Fine aggregate: washed concrete sand, state sand from Bakersfield Ready Mix; meets ASTM C 33.

Coarse aggregate: $9.5-\mathrm{mm}(3 / 8-i n$.$) maximun size crushed granite from Bakersfield Ready Mix;$ meets ASTM C 33, size 8.

Admixture: Protex water-reducing admixture, meets ASTM C 494, type A.

Rec ommended dasage: $2.6 \mathrm{nl} / \mathrm{kg}$ ( $4 \mathrm{fl} \mathrm{oz} / \mathrm{cwt}$ ) of cement.

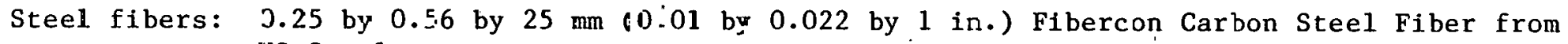
US Steel.

(a) Concrete was batched at Bakersfield Frecast Company in 3 to $4.6 \mathrm{~m}^{3}$ ( 4 to $6 \mathrm{yd}^{3}$ ) batches and was truck nixed for 100 revoluticns. 
TABLE 6 (continued)

B AGGREGATE

\begin{tabular}{|c|c|c|c|}
\hline & \multicolumn{3}{|c|}{ Perzent Passing } \\
\hline & Fine & Soarse & Combined \\
\hline Sieve siza (ASTM C 136) & & & \\
\hline $12.5 \mathrm{~mm}(1 / 2 \mathrm{in.})$ & 100 & 100 & 100 \\
\hline $9.5 \mathrm{~mm}(3 / 8 \mathrm{in.})$ & 100 & 91.9 & 96.4 \\
\hline $4.75 \mathrm{~mm}$ (No. 4) & 99.2 & 5.0 & 56.7 \\
\hline $2.36 \mathrm{~mm}$ (No. 8 ) & 85.0 & 2.0 & 47.6 \\
\hline $1.18 \mathrm{~mm}$ (No. 16) & 66.0 & -- & 30.8 \\
\hline $600 \mu \mathrm{m}$ (No. 30) & 40.6 & -- & 22.3 \\
\hline $300 \mu \mathrm{m}$ (No. 50) & 20.0 & -- & 10.7 \\
\hline $150 \mu \mathrm{m}$ (No. 100$)$ & 7.0 & -- & 3.8 \\
\hline $\begin{array}{l}\text { Amount finer than } 75 \mu \mathrm{m} \\
\text { (No. } 200 \text { sieve) }(\%)\end{array}$ & 2.5 & -- & 1.4 \\
\hline $\begin{array}{l}\text { Specific gravity (ASTM C } 126 \\
\text { and C 127) }\end{array}$ & 2.63 & 2.65 & 2.64 \\
\hline Fineness modulus (ASTM C. 125) & 2.93 & 6.01 & 4.32 \\
\hline $\begin{array}{l}\text { Percentage of each type of } \\
\text { aggregate in mix }\end{array}$ & 55 & 45 & 100 \\
\hline
\end{tabular}


TA3LE 6 (continued)

C. MIX PROPORTIONS $\mathrm{kg} / \mathrm{m}^{3}\left(1 \mathrm{~b} / \mathrm{yd}^{3}\right)$

\begin{tabular}{|c|c|c|c|c|c|c|c|c|}
\hline & \multicolumn{3}{|c|}{. Trial Mix No. } & \multicolumn{2}{|c|}{ Form No. } & \multicolumn{3}{|c|}{ Pane1 No. } \\
\hline & 1 & 2 & 3 & M2 & M3 & PMI-1 & PM1-2 & PM3-1 \\
\hline Cement & $\begin{array}{l}412 \\
(705)\end{array}$ & $\begin{array}{l}418 \\
(705)\end{array}$ & $\begin{array}{l}50 \div \\
(850)\end{array}$ & $\begin{array}{l}418 \\
(705)\end{array}$ & $\begin{array}{l}326 \\
(550)\end{array}$ & $\begin{array}{l}418 \\
(705)\end{array}$ & $\begin{array}{l}418 \\
(705)\end{array}$ & $\begin{array}{l}418 \\
(705)\end{array}$ \\
\hline $\mathrm{CaCl}_{2}$ & -- & -- & -- & $\begin{array}{l}4.2 \\
(7.1)\end{array}$ & -- & $\begin{array}{l}4.2 \\
(7.1)^{\prime}\end{array}$ & $\begin{array}{l}4.2 \\
(7.1)\end{array}$ & -- \\
\hline Water & (b) & (b) & (b) & (b) & (b) & (b) & (b) & (b) \\
\hline Fine aggregate & $\begin{array}{l}925 \\
(1560)\end{array}$ & $\begin{array}{l}925 \\
(1560)\end{array}$ & $\begin{array}{l}925 \\
(1560)\end{array}$ & $\begin{array}{l}925 \\
(1560)\end{array}$ & $\begin{array}{l}1008 \\
(1700)\end{array}$ & $\begin{array}{l}9251 \\
(1560)\end{array}$ & $\begin{array}{l}925 \\
(1560)\end{array}$ & $\begin{array}{l}925 \\
(1560)\end{array}$ \\
\hline Coarse aggregate & $\begin{array}{l}765 \\
(1290)\end{array}$ & $\begin{array}{l}765 \\
(1290)\end{array}$ & $\begin{array}{l}7 € .5 \\
(12 \leq 0)\end{array}$ & $\begin{array}{l}765 \\
(1290)\end{array}$ & $\begin{array}{l}1008 \\
(1700)\end{array}$ & $\begin{array}{l}76 j \\
(1290 j\end{array}$ & $\begin{array}{l}765 \\
(1290)\end{array}$ & $\begin{array}{l}765 \\
(1290)\end{array}$ \\
\hline Steel fibers & -- & $\begin{array}{l}71 \\
(120)\end{array}$ & $\begin{array}{l}71 \\
(1 \approx C)\end{array}$ & -- & -- & -- & $\begin{array}{l}53 \\
(90)\end{array}$ & -- \\
\hline Admixture & $\begin{array}{l}1.1 \\
(2.4)\end{array}$ & $\begin{array}{l}1.1 \\
(2.4)\end{array}$ & $\begin{array}{l}1.3 \\
(2.9)\end{array}$ & $\begin{array}{l}1.1 \\
(2.4)\end{array}$ & $\begin{array}{l}0.9 \\
(1.9)\end{array}$ & $\begin{array}{l}1.1 \\
(2.4)\end{array}$ & $\begin{array}{l}1.1 \\
(2.4)\end{array}$ & $\begin{array}{l}1.1 \\
(2.4)\end{array}$ \\
\hline
\end{tabular}

(b) Approximately $136 \mathrm{~kg}$ (300 1b); water. content adjusted to produce cesired slump. 
TABLE 6 (continued)

D. CONCRETE CASTINGS

\begin{tabular}{|c|c|c|c|c|c|c|c|c|}
\hline & \multicolumn{3}{|c|}{ Trial Mix No. } & \multicolumn{2}{|c|}{ Form No. } & \multicolumn{3}{|c|}{ Panel No. } \\
\hline & 1 & 2 & 3 & M2 & M3 & PM1-1 & PM1-2 & PM3-1 \\
\hline Date cast & $1 / 3 / 80$ & $1 / 14 / 80$ & $1 / 28 / 80$ & $3 / 7 / 80$ & $3 / 14 / 80$ & $3 / 8 / 80$ & $3 / 12 / 80$ & $3 / 18 / 80$ \\
\hline Time cast & $1 \mathrm{E} \cdot \mathrm{M}$ & 10 A.M. & 2 P.M. & 2 P.M. & 2:3C P.M. & 3:30 P.M. & 8:30 A.M. & 2:30 P.M. \\
\hline Date stripped & $1 / 4 / 80$ & $1 / 15 / 80$ & $1 / 29 / 80$ & $3 / 8 / 80$ & $3 / 1 \pi / 80$ & $3 / 11 / 80$ & $3 / 13 / 80$ & $3 / 19 / 80$ \\
\hline Steaming time $(c)(h)$ & 8 & 8 & 8 & 20 & 10 & 10 & 12.5 & 14 \\
\hline $\begin{array}{l}\text { Maximum temperature }\left[{ }^{\circ} \mathrm{C}\right. \\
\left({ }^{\circ} \mathrm{F}\right)\end{array}$ & $\begin{array}{l}49 \\
(120)\end{array}$ & $\begin{array}{l}49 \\
(120)\end{array}$ & $\begin{array}{l}49 \\
(120)\end{array}$ & $\begin{array}{l}49 \\
(120)\end{array}$ & $\begin{array}{l}49 \\
(120)\end{array}$ & $\begin{array}{l}49 \\
(120)\end{array}$ & $\begin{array}{l}49 \\
(120)\end{array}$ & $\begin{array}{l}77 \\
(170)\end{array}$ \\
\hline Cement type & II & II & $\mathrm{v}$ & II & II & II & II & II \\
\hline $\begin{array}{l}\text { Maximum aggregate }[\mathrm{mm} \\
\text { (in.)] }\end{array}$ & $\begin{array}{l}9.5 \\
(3 ! 8)\end{array}$ & $\begin{array}{l}9.5 \\
(3 / 8)\end{array}$ & $\begin{array}{l}9.5 \\
(3 / 8)\end{array}$ & $\begin{array}{l}9.5 \\
(3 / 8)\end{array}$ & $\begin{array}{l}25.4 \\
(1.0)\end{array}$ & $\begin{array}{l}9 . .5 \\
(3 / 8)\end{array}$ & $\begin{array}{l}9.5 \\
(3 / 8)\end{array}$ & $\begin{array}{l}9.5 \\
(3 / 8)\end{array}$ \\
\hline \multicolumn{9}{|c|}{ E. FRESH CONCRETE } \\
\hline & \multicolumn{3}{|c|}{ Trial Mix No. } & \multicolumn{2}{|c|}{ Form No. } & \multicolumn{3}{|c|}{ Panel No. } \\
\hline & 1 & 2 & 3 & M2 & M3 & PM1-1 & PM1-2 & PM3-1 \\
\hline Slump [mm (in.)] & $\begin{array}{l}90 \\
(3.5)\end{array}$ & $\begin{array}{l}100 \\
(4)\end{array}$ & $\begin{array}{l}75 \\
(3)\end{array}$ & $\begin{array}{l}50 \\
(2)\end{array}$ & -- & $\begin{array}{l}57 \\
(2.25)\end{array}$ & $\begin{array}{l}90 \\
(3.5)\end{array}$ & $\begin{array}{l}100 \\
(4)\end{array}$ \\
\hline $\begin{array}{l}\text { Unit weight }\left[\mathrm{kg} / \mathrm{m}^{3}\right. \\
\left.\quad\left(1 \mathrm{~b} / \mathrm{ft}^{3}\right)\right]\end{array}$ & $\begin{array}{l}2350 \\
(147)\end{array}$ & $\begin{array}{l}2350 \\
(147)\end{array}$ & $\begin{array}{l}2450 \\
(153)\end{array}$ & -- & -- & -- & -- & -- \\
\hline $\begin{array}{l}\text { Concrete temperature }\left[{ }^{\circ} \mathrm{C}\right. \\
\left.\left({ }^{\circ} \mathrm{F}\right)\right]\end{array}$ & $\begin{array}{l}18 \\
(64)\end{array}$ & $\begin{array}{l}22 \\
(72)\end{array}$ & $\begin{array}{l}18 \\
(64)\end{array}$ & $\begin{array}{l}22 \\
(71)\end{array}$ & -- & $\begin{array}{l}26 \\
(78)\end{array}$ & $\begin{array}{l}16 \\
(61)\end{array}$ & $\begin{array}{l}20 \\
(68)\end{array}$ \\
\hline $\begin{array}{l}\text { Ambient temperature }\left[{ }^{\circ} \mathrm{C}\right. \\
\left.\left({ }^{\circ} \mathrm{F}\right)\right]\end{array}$ & $\begin{array}{l}16 \\
(60)\end{array}$ & $\begin{array}{l}21 \\
(70)\end{array}$ & $\begin{array}{l}16 \\
(60)\end{array}$ & $\begin{array}{l}21 \\
(70)\end{array}$ & -- & $\begin{array}{l}24 \\
(75)\end{array}$ & $\begin{array}{l}14 \\
(58)\end{array}$ & $\begin{array}{l}18 \\
(65)\end{array}$ \\
\hline
\end{tabular}

(c) $\triangle$ bove $38^{\circ} \mathrm{C}\left(100^{\circ} \mathrm{F}\right)$. 
TABLE 6' (continued)

F. COMPRESSIVE STRENGTI [MPa (psï)] OE 152 BY 305 mm (6 BY 12 in.) CYLINDERS PER ASTM C 39

\begin{tabular}{|c|c|c|c|c|c|c|c|c|}
\hline \multirow[b]{2}{*}{ Age (days) } & \multicolumn{3}{|c|}{ Trial Mix No. } & \multicolumn{2}{|c|}{ Form No. } & \multicolumn{3}{|c|}{ Panel No: } \\
\hline & 1 & 2 & 3 & $\mathrm{M} 2$ & M3 & PM1-1 & PM1-2 & PM3-1 \\
\hline 1 & $\begin{array}{l}(4.2 \\
(2070)\end{array}$ & $\begin{array}{l}14.8 \\
(2160)\end{array}$ & $\begin{array}{l}11.9 \\
(1730)\end{array}$ & -- & -- & -- & $\begin{array}{l}18.3 \\
(2670)\end{array}$ & $\begin{array}{l}40.1 \\
(5850)\end{array}$ \\
\hline 3 & -- & -- & -- & $\begin{array}{l}35.7(d) \\
(5290)\end{array}$ & -- & $\begin{array}{l}37.6 \\
(5480)\end{array}$ & - & -- \\
\hline 7 & $\begin{array}{l}29.8 \\
(4340)\end{array}$ & $\begin{array}{l}24.7 \\
(3600)\end{array}$ & $\begin{array}{l}20.1 \\
(2930)\end{array}$ & $\begin{array}{l}39.9 \\
(5820)\end{array}$ & $\begin{array}{l}25.7 \\
(3750)\end{array}$ & $\begin{array}{l}43.1 \\
(6290)\end{array}$ & $\begin{array}{l}33.8 \\
(4930)\end{array}$ & $\begin{array}{l}48.5 \\
(7080)\end{array}$ \\
\hline 14 & -- & -- & $\begin{array}{l}23.3 \\
(3400)\end{array}$ & -- & $\begin{array}{l}31.2 \\
(4550)\end{array}$ & -- & -- & $\begin{array}{l}52.1 \\
(7600)\end{array}$ \\
\hline 21 & -- & -- & -- & -- & $\begin{array}{l}31.9 \\
(4650)\end{array}$ & $\begin{array}{l}51.9 \\
(7570)\end{array}$ & $\begin{array}{l}38.2 \\
(5570)\end{array}$ & $\begin{array}{l}48.5 \\
(7080)\end{array}$ \\
\hline 28 & - & $\begin{array}{l}33.5 \\
(4890)\end{array}$ & $\begin{array}{l}25.2 \\
i 3675\end{array}$ & -- & -- & -- & -- & -- \\
\hline
\end{tabular}

G. FLEXURAL STRENGTH OF 152 EY 152 EY $508 \mathrm{~mm}$ ( 6 BY 6 BY 20 in.) BEAMS PER ASTM C 78. MODULUS OF RUPTURE [MPa (psi)] THIRD POINT LOADING

\begin{tabular}{|c|c|c|c|c|c|c|c|c|}
\hline \multirow[b]{2}{*}{ (days) } & \multicolumn{3}{|c|}{ Trial Mix No. } & \multicolumn{2}{|c|}{ Form No. } & \multicolumn{3}{|c|}{ Panel No. } \\
\hline & 1 & 2 & 3 & M2 & M3 & PM1-1 & PM1 -2 & PH3-1 \\
\hline 7 & -- & $\begin{array}{l}\angle .0 \\
(580)\end{array}$ & $\begin{array}{l}4.5 \\
(660)\end{array}$ & -- & -- & -- & $\begin{array}{l}4.4 \\
(640)\end{array}$ & $\begin{array}{l}4.1 \\
(600)\end{array}$ \\
\hline 28 & -- & $\begin{array}{l}5.4 \\
(790)\end{array}$ & $\begin{array}{l}5.4 \\
(785)\end{array}$ & -- & -- & -- & -- & -- \\
\hline
\end{tabular}

(d) At age of 4 deys. 
TABLE 7

SUMMIARY OF LASER INSPECTIONS

\begin{tabular}{|c|c|c|c|c|c|c|}
\hline $\begin{array}{c}\text { Inspection } \\
\text { No. }\end{array}$ & Date & $\begin{array}{c}\text { Form/ } \\
\text { Panel No. }\end{array}$ & Orientation & Position & Slat & Remarks \\
\hline 1 & $3 / 4 / 80$ & $M 1$ & Convex & $1,2,3$ & All & (a) \\
\hline 2 & $3 / 8 / 80$ & $M 2$ & Concave & $1,2,3$ & (b) & (a) \\
\hline 3 & $3 / 11 / 80$ & M1 & Convex & 1 & (b) & $(a, c)$ \\
\hline 4 & $3 / 11 / 80$ & PM1-1 & Concave & 1 & (b) & (a) \\
\hline 5 & $3 / 14 / 80$ & PM1-2 & Concave & $1,2,3$ & (b) & (a) \\
\hline 6 & $3 / 20 / 80$ & PM3-1 & Concave & $1,2,3$ & (b) & (a) \\
\hline 7 & $3 / 26 / 80$ & PMI-2 & Supports & $1,2,3$ & (b) & $(a, c)$ \\
\hline 8. & $3 / 26 / 80$ & PM3-1 & Supports & $1,2,3$ & (b) & $(a, c)$ \\
\hline 9 & $4 / 1 / 80$ & PMI -2 & Supports & $1,2,3$ & (b) & $(a, c)$ \\
\hline 10 & $4 / 1 / 80$ & PM3-1 & Supports & $1,2,3$ & (b) & $(a, c)$ \\
\hline 11 & $4 / 7 / 80$ & M3 & Convex & $1,2,3$ & (d) & (a) \\
\hline $12 a$ & $4 / 3 / 80$ & $M 2$ & Concave & 1 & $\mathrm{All}$ & (a) \\
\hline $12 b$ & $4 / 9 / 80$ & M2 & Concave & 2 & Al1 & (a) \\
\hline $12 c$ & $4 / 10 / 80$ & $M 2$ & Concave & 3 & All & (a) \\
\hline $13 a$ & $4 / 14 / 80$ & MI & Convex & 3 & Al1 & \\
\hline $13 b$ & $4 / 14 / 80$ & M1 & Convex & 2 & Al1 & \\
\hline $13 c$ & $4 / 15 / 80$ & MI & Convex & 1 & A11 & \\
\hline
\end{tabular}

(a) Two readings taken per slat.

(b) Slats $1,9,18,27,36,45,54,63$, and 71 .

(c) Results not presented owing to insufficient data.

(d) Slats $1,3,6,9 \ldots 69$ (in increments of 3 ), and 71 . 
TABLE 8

DIFFERENCE BETWEEN ACHIEVED AND DESIRED SLAT ANGLES

\begin{tabular}{|c|c|c|c|c|c|c|}
\hline \multirow[b]{2}{*}{ Panel(a)/Form } & \multirow{2}{*}{$\begin{array}{l}\text { Inspection } \\
\text { No. }\end{array}$} & \multirow{2}{*}{$\begin{array}{l}\text { No. of } \\
\text { Valid } \\
\text { Readings }\end{array}$} & \multicolumn{4}{|c|}{$\begin{array}{c}\text { Achieved Minus Desired } \\
\text { Anglo (dog) }\end{array}$} \\
\hline & & & Average & $\sigma^{(b)}$ & $2 \sigma^{(b)}$ & $3 \sigma(b)$ \\
\hline Initial steel form $M 1$ & 1 & 209 & +0.10 & 0.13 & 0.26 & 0.38 \\
\hline Final steel form $M I$ & 13 & 208 & +0.09 & 0.09 & 0.18 & $0.28(c)$ \\
\hline $\begin{array}{l}\text { Initial concrete form } \\
\text { M2 }\end{array}$ & 2 & 27 & -0.04 & $0.12^{\circ}$ & 0.23 & 0.35 \\
\hline $\begin{array}{l}\text { Final concrete form } \\
\text { M2 }\end{array}$ & 12 & 213 & -0.09 & 0.15 & 0.29 & 0.44 \\
\hline $\begin{array}{l}\text { Final concrete form } \\
\text { M3 }\end{array}$ & 11 & 57 & +0.17 & 0.20 & 0.40 & $0.60(d)$ \\
\hline Initial panel PM1-1 & 4 & 8 & 0 & 0.11 & 0.23 & 0.34 \\
\hline Initial panel PM1-2 & 5 & 27 & -0.08 & 0.10 & 0.19 & 0.29 \\
\hline $\begin{array}{l}\text { PM1-2, } 8 \text { days on } \\
\text { supports }\end{array}$ & 7 & (e) & (e) & (e) & (e) & (e) \\
\hline $\begin{array}{l}\text { PM1-2, } 14 \text { days on } \\
\text { oupports }\end{array}$ & 9 & (e) & (e) & (e) & (e) & (e) \\
\hline Initial panel PM3-1 & 6 & 20 & -0.02 & 0.14 & 0.28 & 0.41 \\
\hline $\begin{array}{l}\text { PM3-1, } 2 \text { days on } \\
\text { supports }\end{array}$ & 8 & (c) & (e) & (e) & (e) & (e) \\
\hline $\begin{array}{l}\text { PM3-1, } 8 \text { days on } \\
\text { supports }\end{array}$ & 10 & (e) & (e) & (e) & (e) & (e) \\
\hline
\end{tabular}

(a) Panel is $1.83 \mathrm{~m}$ ( $6 \mathrm{ft}$ ) long with a $3.6-\mathrm{m}$ (11 ft $10 \mathrm{in.}$ ) aperture and 115-m (4.5-in.) thickness.

(b) $\sigma=$ standard deviation. Under the standard normal dietribution curve, $68.27 \%$ of the angle differences fall within to from the average, $95.45 \%$ fall within $+2 \sigma$ from the average, and $99.75 \%$ fall within $+3 \sigma$ from the average.

(c) Can be shimmed to 0.10 deg.

(d) Fiberglass-cuated concrete form M3 had many surface irregularities from voids in concrete form $M 2$. The $3 \sigma$ value can be improved by casting $M 3$ against a smooth steel or fiberglass surface.

(e) Insufficient data. 
TABLE 9

COMPARISON OF SLAT ANGLES ON PANELS AND FORMS

\begin{tabular}{|c|c|c|c|c|c|c|}
\hline \multirow[b]{2}{*}{ Difference Between } & \multirow{2}{*}{$\begin{array}{l}\text { Inspection } \\
\text { No. }\end{array}$} & \multirow{2}{*}{$\begin{array}{c}\text { No. of } \\
\text { Valid } \\
\text { Readings }\end{array}$} & \multicolumn{4}{|c|}{$\begin{array}{c}\text { Difference in Angle } \phi \\
\text { (deg) }\end{array}$} \\
\hline & & & Average & $\sigma$ & $2 \sigma$ & 30 \\
\hline Initial and final $M 1$ & $1-13$ & 207 & 0.01 & 0.14 & 0.27 & 0.41 \\
\hline Initial and final M2 & $2-12$ & 27 & 0.03 & 0.12 & 0.25 & 0.37 \\
\hline Initial M1 and initial M2 & $1-2$ & 27 & 0.17 & 0.18 & 0.35 & 0.53 \\
\hline Final $M 1$ and final $M 2$ & $13-12$ & 201 & 0.17 & 0.14 & 0.29 & 0.43 \\
\hline Final $M 1$ and initial $\mathrm{PMI}-1$ & $13-4$ & 8 & 0.11 & 0.14 & 0.28 & 0.42 \\
\hline Final ML ànd inirial Plyl-2 & $13-5$ & 27 & 0.18 & 0.1 .5 & 0.29 & 0.44 \\
\hline Final $M 3$ and initial PM3-1 & $11-6$ & 19 & 0.12 & 0.17 & 0.35 & 0.52 \\
\hline Final $M 3$ and final $M 2$ & $11-12$ & 57 & 0.23 & 0.20 & 0.41 & 0.62 \\
\hline $\begin{array}{l}\text { Initial M2 and initial } \\
\text { PM1-1 }\end{array}$ & $2-4$ & 8 & -0.01 & 0.07 & 0.14 & 0.21 \\
\hline $\begin{array}{l}\text { Initial M2 and initial } \\
\text { PM1-2 }\end{array}$ & $2-5$ & 27 & 0.05 & 0.09 & 0.19 & 0.28 \\
\hline $\begin{array}{l}\text { Initial PM1-1 and initial } \\
\text { PM1-2 }\end{array}$ & $4-5$ & 8 & 0.04 & 0.07 & 0.14 & 0.20 \\
\hline $\begin{array}{l}\text { Initial PM1-1 and initial } \\
\text { PM3-1 }\end{array}$ & $4-6$ & 6 & 0.03 & 0.12 & 0.24 & 0.36 \\
\hline $\begin{array}{l}\text { Initial PM1-2 and initial } \\
\text { PM3-1 }\end{array}$ & $5-6$ & 27 & -0.11 & 0.22 & 0.44 & 0.66 \\
\hline
\end{tabular}


overall, but was not entirely satisfactory in rainy weather nor during steam curing above $49^{\circ} \mathrm{C}\left(120^{\circ} \mathrm{F}\right)$. Additional tests should be conducted during phase II to find the best available parting compound for the type of forms and curing conditions selected.

All the sealing compounds tested were acceptable. Thompson's water seal was selected for the panels because it was the easiest to obtain.

The most important coating tests dealt with surface hardeners for concrete forms. To be effective, such forms must have a smooth, hard aurface and must be capable of being used 30 to 40 times without major refurbishing. Several products and methods of application were tested, with the most successful product being a fiberglass surface with concrete backing. In the fabrication of the small form M3, a 6-mm (0.25-in.) thick fiberglass layer was directly applied to a concrete mold (M2) which had previously been coated with a release agent. The fiberglass was coated with epoxy glue, covered with a 100-mm (4-in.) thick concrete backing, and steam cured overnight. This produced a very smooth surface, which was judged to be good for at least 40 reuses with prnper sare.

Epoxy-coated concrete forms fabricated in the same manner as the fiberglass-coated form were not acceptable. The epoxy became soft under steam curing, form M3 would not separate from mold M2, and the epoxy was much more expensive than the fiberglass.

Concrete forms on which a thin layer of hardener was sprayed or brushed were aiso unacceptable. Even though the surface hardness and release properties were good, the cuating could not be applied uniformly enough to produce a smooth surtace for the mirrors.

\subsubsection{Stee1 Form M1}

Figure 10 shows steel form $M$ during assembly and in its final form; Fig. 11 shows the form details. The form was designed with stability as the 


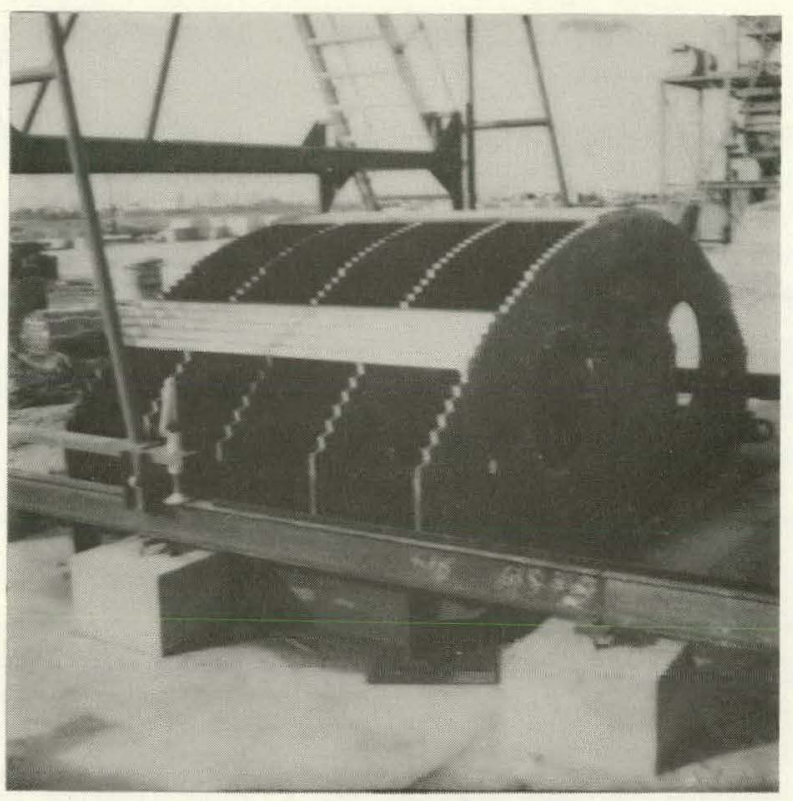

(a)

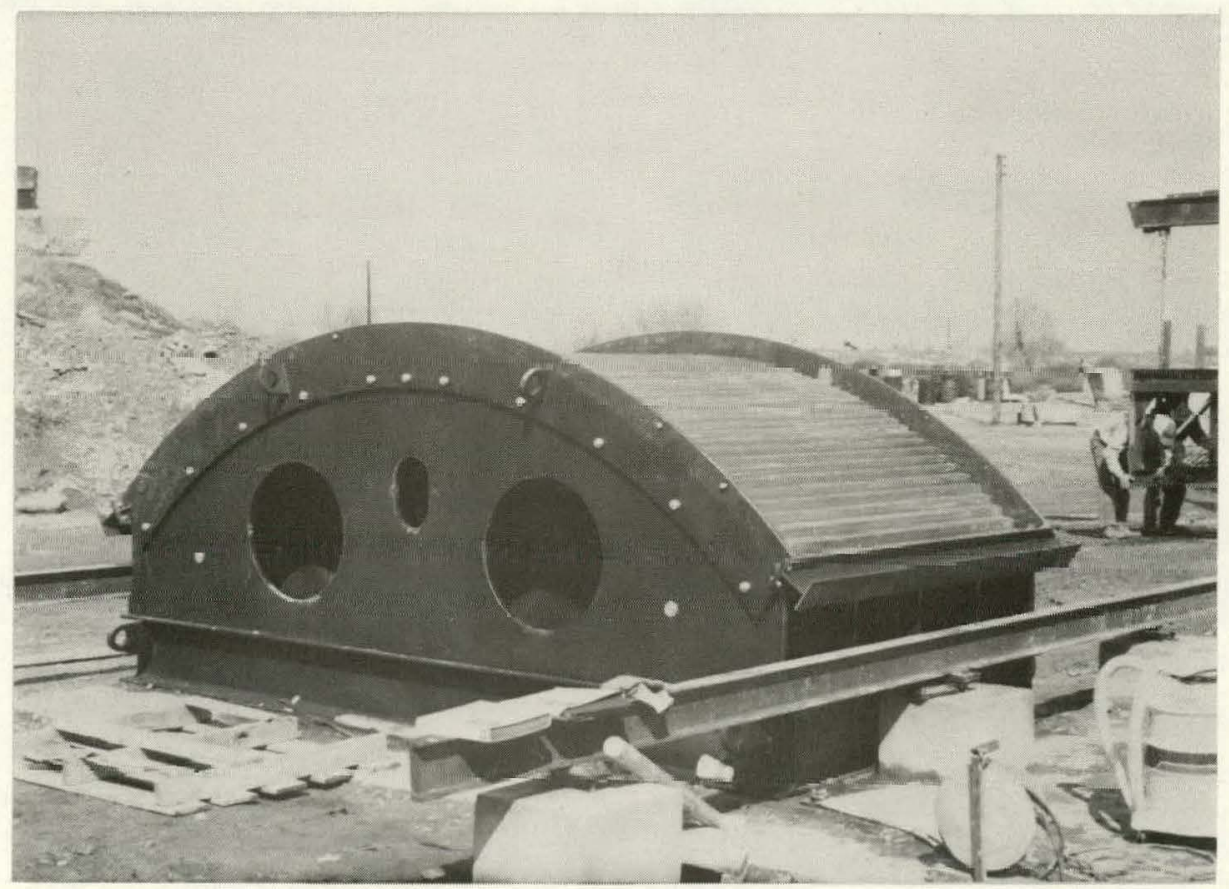

(b)

Fig. 10. Steel form MI: (a) during assembly; (b) assembled 


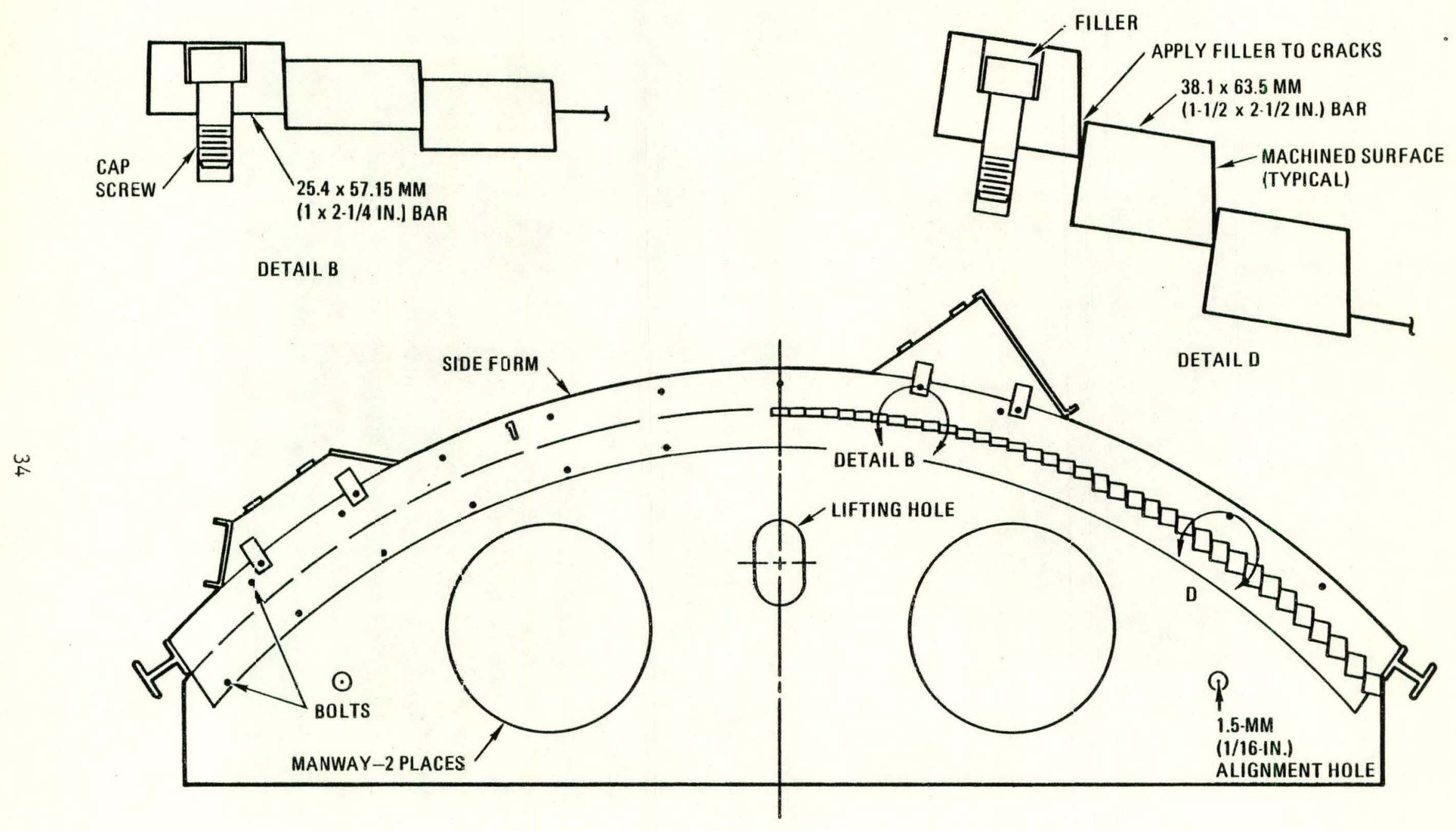

Fig. 11. Letails of steel form M1 
main feature and with provisions for adjusting the slat angles as the second feature. It consists of seventy-one 1.83-m (6-ft) long cold-rolled steel bars bolted to five $25.4-\mathrm{mm}$ ( 1 -in.) thick steel bulkheads. The bulkheads were flame cut within $6 \mathrm{~mm}(0.25 \mathrm{in.})$ of the desired shape and precisely machined to within $+0.10 \mathrm{deg}$ of the desired slat angle.

The bars were machined on only one side for the draft angle. The remaining three sides were sufficiently smooth from cold rolling and required no machining. All the bars were deburred, and many of the lower corners on the machined side had to be ground for proper fit. This was done in the field with a hand-held grinding disc which nicked some of the vertical slat surfaces. Later on, the nicked surfaces caused poor stripping of the concrete from the form and breakage of the delicate slat corners. Many of the bars had up to a 3-mm (1/8-in.) bow from the relieved stresses on the machined surface, and some had a slight twist (up to about $0.40 \mathrm{deg}$ ).

The bulkheads were aligned by shining a laser beam through $1.5-\mathrm{mm}$ (1/16-in.) alignment holes and bolted to the skid. The slats were then bolted to the bulkheads. Quite a bit of effort was required to straighten the bowed slats.

The initial slat alignment was checked at over 200 locations with the GA laser inspection fixture. The average difference between the achieved and the desired angle was $+0.10 \mathrm{deg}$, with $99.75 \%$ of the slats within $+0.38 \mathrm{deg}$ of the average. The slats cnuld have heen shimmed to within \pm 0.10 deg of the desired value; however, this was not done owing to lack of time, particularly because the concrete forms and panels had to be cast at once. Furthermore, there was some question as to the accuracy of the measurements since all the readings were taken during a rainstorm and the inspectors were still learning how to use the laser inspection fixture. Section 4.3.7 discusses the measurements in more detail.

The bolt holes were filled with Bondite premium body filler mixed with Marson cream hardener. The body filler was then sanded down and the cracks hetween the slats filled with Bondite and Devcon plastic steel. 
After all the castings, form alignment was again checked through the alignment holes and was found to be satisfactory, despite rough handling, vibrations, and steam curing. The slat angle measurements were repeated and six representative slats were successfully shimmed to within +0.10 deg of the desired angle to demonstrate the feasibility of the shimming operation. In general, the steel form performed very well, except for the nicked areas.

The form could be improved by tightening the bar length tolerance to $\pm 0.5 \mathrm{~mm}(0.02 \mathrm{in.})$ so that there is no gap between the side plate and the bars. The steel form for production should he assembled and checked at the place of fabrication, where proper equipment and skilled personncl are avail-able. The Bondite putty and Devcon plastic steel must be sanded to specified tolerances. When the steel form is not in use, it must be covered with grease for protection against corrosion. The only significant disadvantages of the steel form are its high initial cost and the long lead time needed for fabrication.

\subsubsection{Concrete Form M2}

Concrete form M2 was initially going to be cast with a fiberglass coating which was the same type as that for M3 (see Section 4.3.4). However, this would have delayed the schedule by another week and was not acceptable. To save time, the concrete was directly cast against steel form M1, over a parting compound and without fiberglass. Figure 12 depicts how the form was fabricated, and Fig. 13 shows the outline of the concrete forms.

The concrete was not vibrated well enough and voids developed in several areas. In other areas, especially where the steel slats were ground in the field, some of the concrete corners broke during stripping. These broken areas were patched with Pour Stone quick setting cement, but the patching was inadequate. The voids and patched corners created some problems during manufacture of M3. 


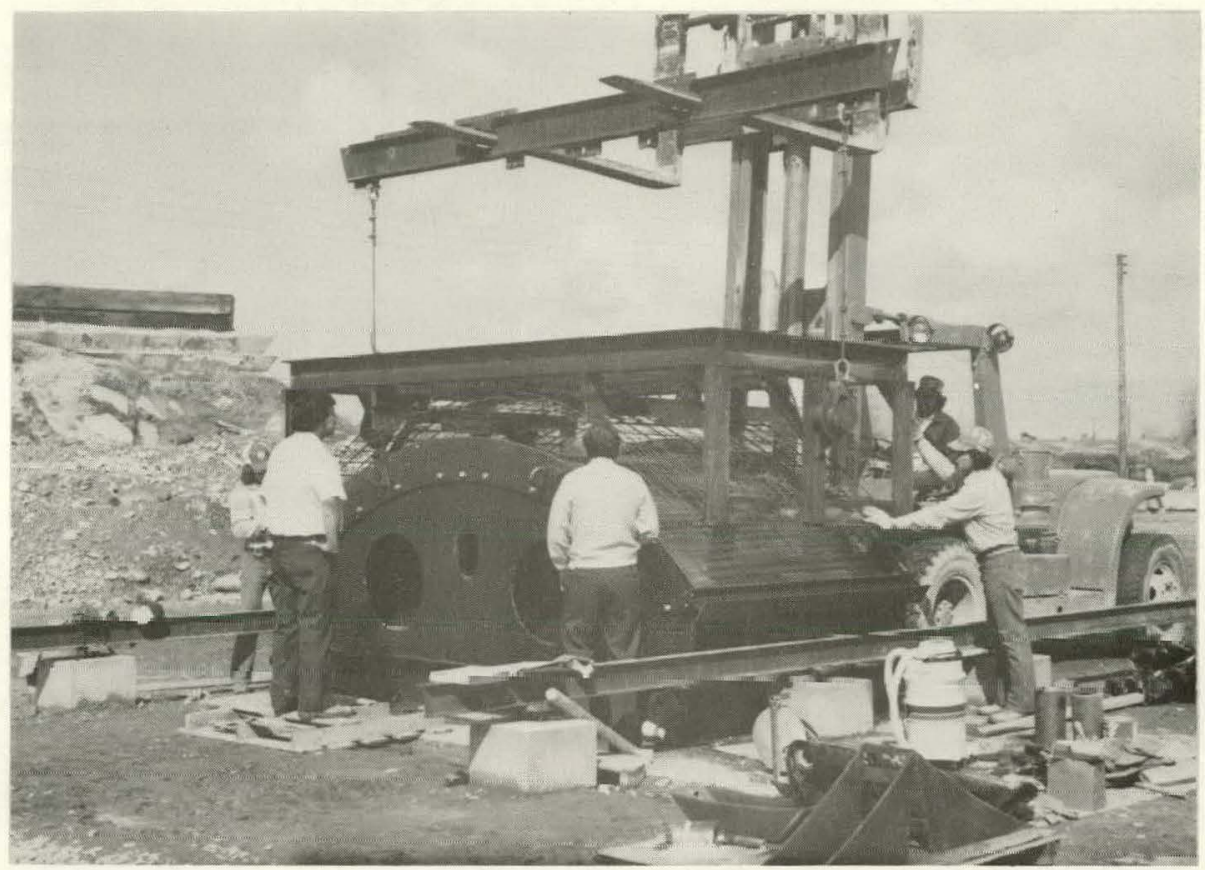

(a)

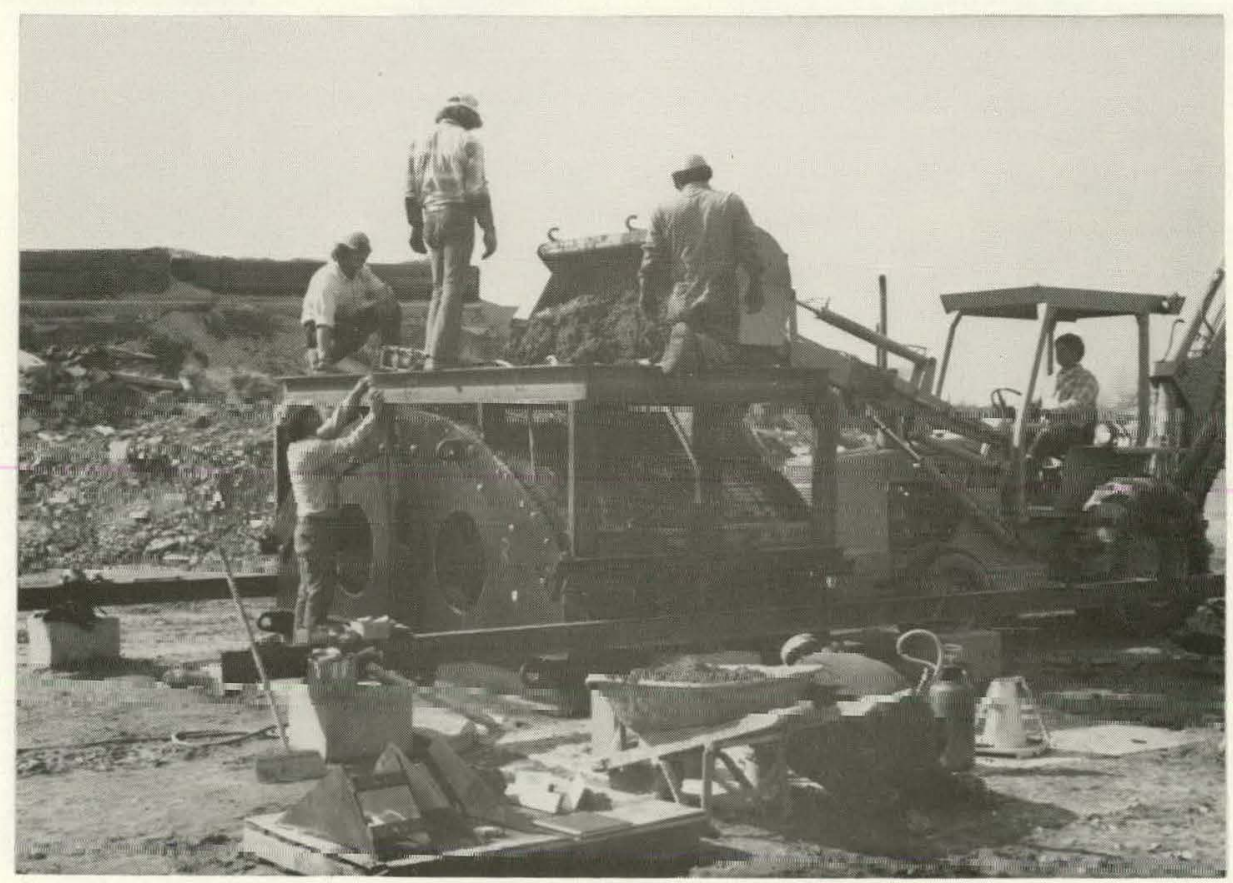

(b)

Fig. 12. Fabrication of concrete form M2: (a) installation of stee1 frame and reinforcement cage; (b) placement of concrete 


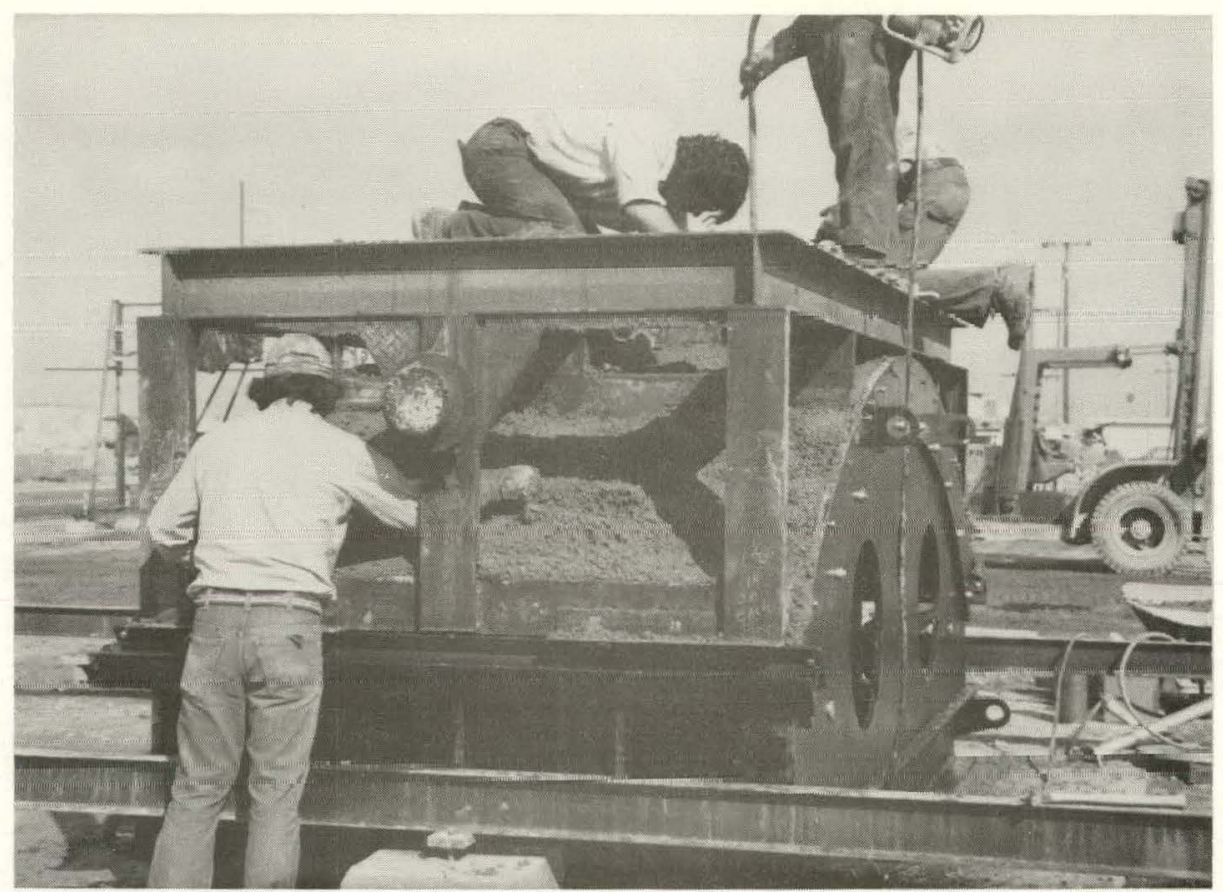

(c)

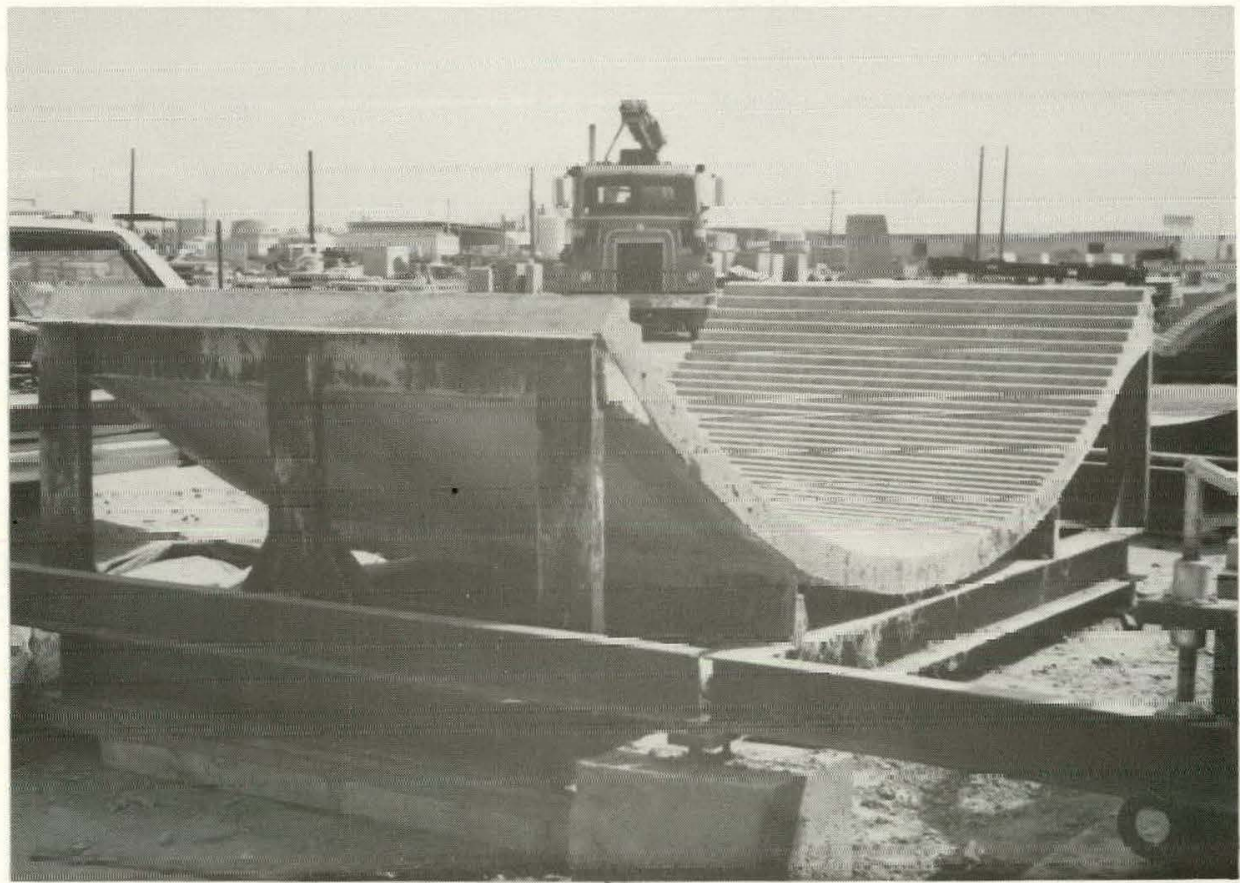

(d)

Fig. 12. Fabrication of concrete form M2: (c) vibration of concrete; (d) final product 

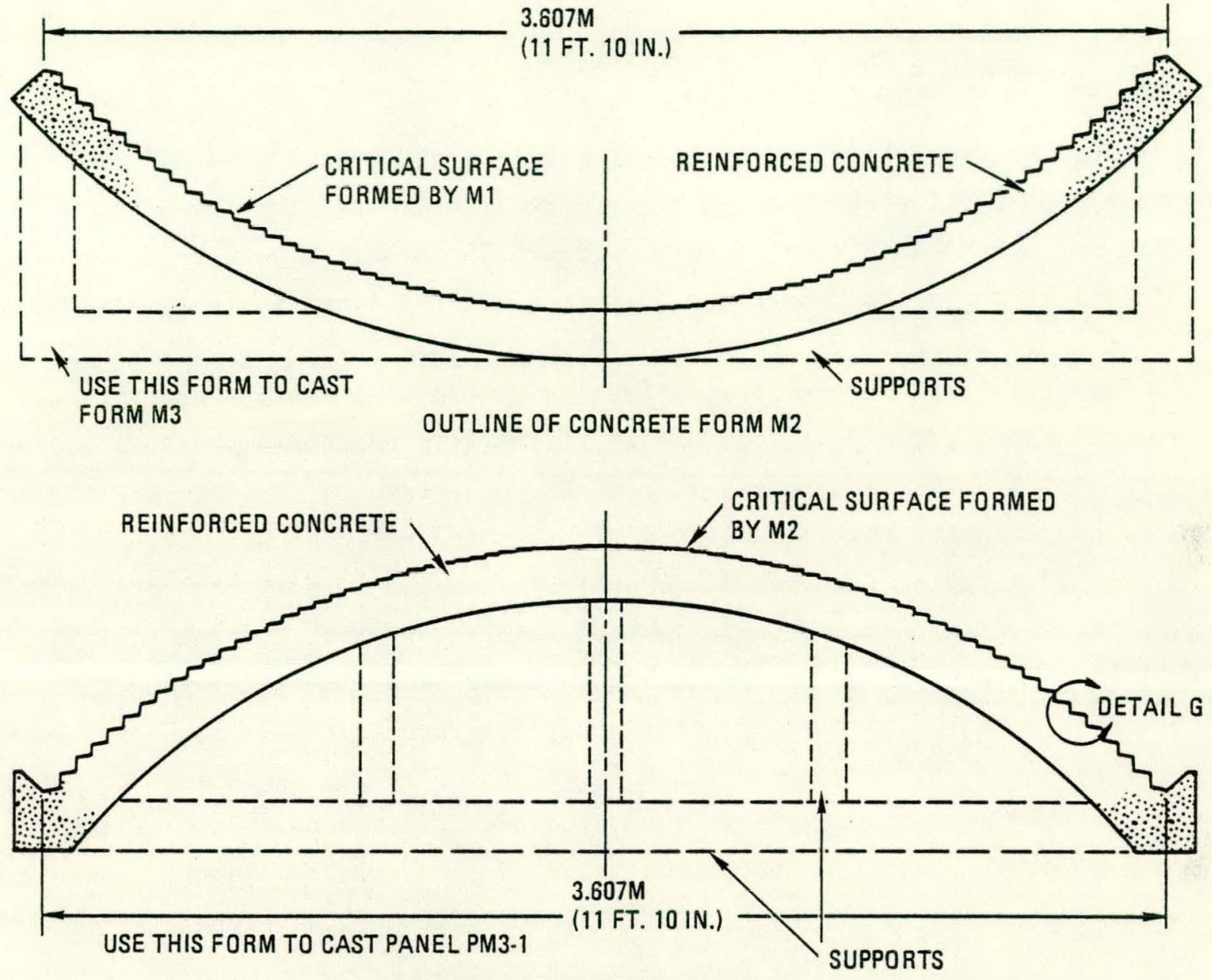

OUTLINE OF CONCRETE FORM M3

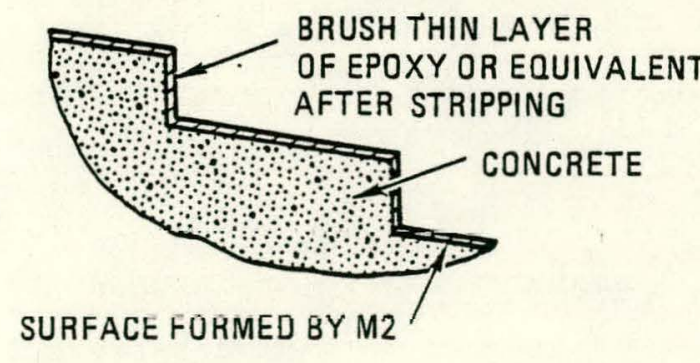

OPTION 1

DETAIL G

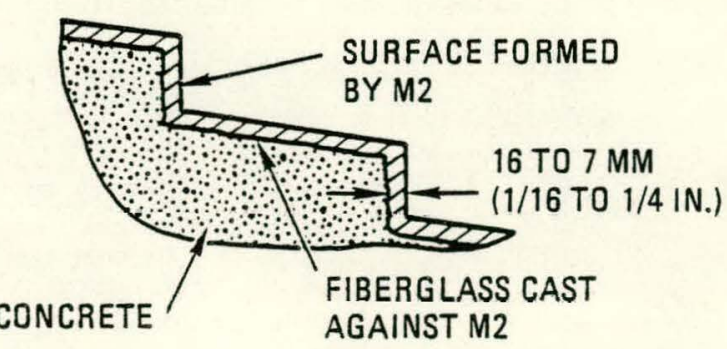

OPTION 2

Fig. 13. Outline of concrete forms 
Overall, the form reproduced the angles quite well (see Section 4.3.7). The form did not warp or crack, and the steel framework of M2 seemed to be rigid enough and the reinforcing strong enough.

\subsubsection{Fiberglass-Coated Concrete Form M3}

Form M3 was fabricated by shooting a 6-mm (0.25-in.) layer of fiberglass onto M2 and placing concrete on top of the fiberglass. The steel support frame was embedded in the concrete. Figure 13 shows the outline of form M3, and Fig. 14 shows the fiberglass operation and the final produrt.

Concrete form M2 was first liberally coated with Finish Kare Blue Wax using a sponge. The paste wax had a fairly stiff consistency and filled most of the voids in the concrete surface. The next day, M2 was sprayed with a thin coat of black Tooling Jell Coat. After the gel coat was dry, the 6-mm (0.25-in.) layer of fiberglass was applied in approximately 12 coats. The glass rope, Erskine Johns-Chop, was fed into a gun which chopped it into 25$\mathrm{mm}$ ( 1-in.) lengths and sprayed on M2 together with the resin, Kopper's Resin 6641 (Fig. 14a). Immediately after spraying, each layer was compacted wilh a small hand roller. Atter the sixth layer, the fiberglass was left to harden, and then the next six layers were sprayed on. Next, a thin coat of 1001 LPL epoxy glue was brushed on the fiberglass. Fifteen minutes later, concrete was placed on top of the glue. After steam curing, M3 was separated from M2, rotated, and set up to cast panel PM3-1.

The fiberglass surface of M3 had the imperfections of M2, but there was enough good surface present to demonstrate that this type of form could do the job.

There was a loose 100-mm (4-in.) strip of fiberglass around the entire periphery of M3 where no 1001 LPL epoxy glue had been used between the concrete and the fiberglass. The glue was purposely omitted from this area because it was feared that the glue might work its way between M2 and M3 and 


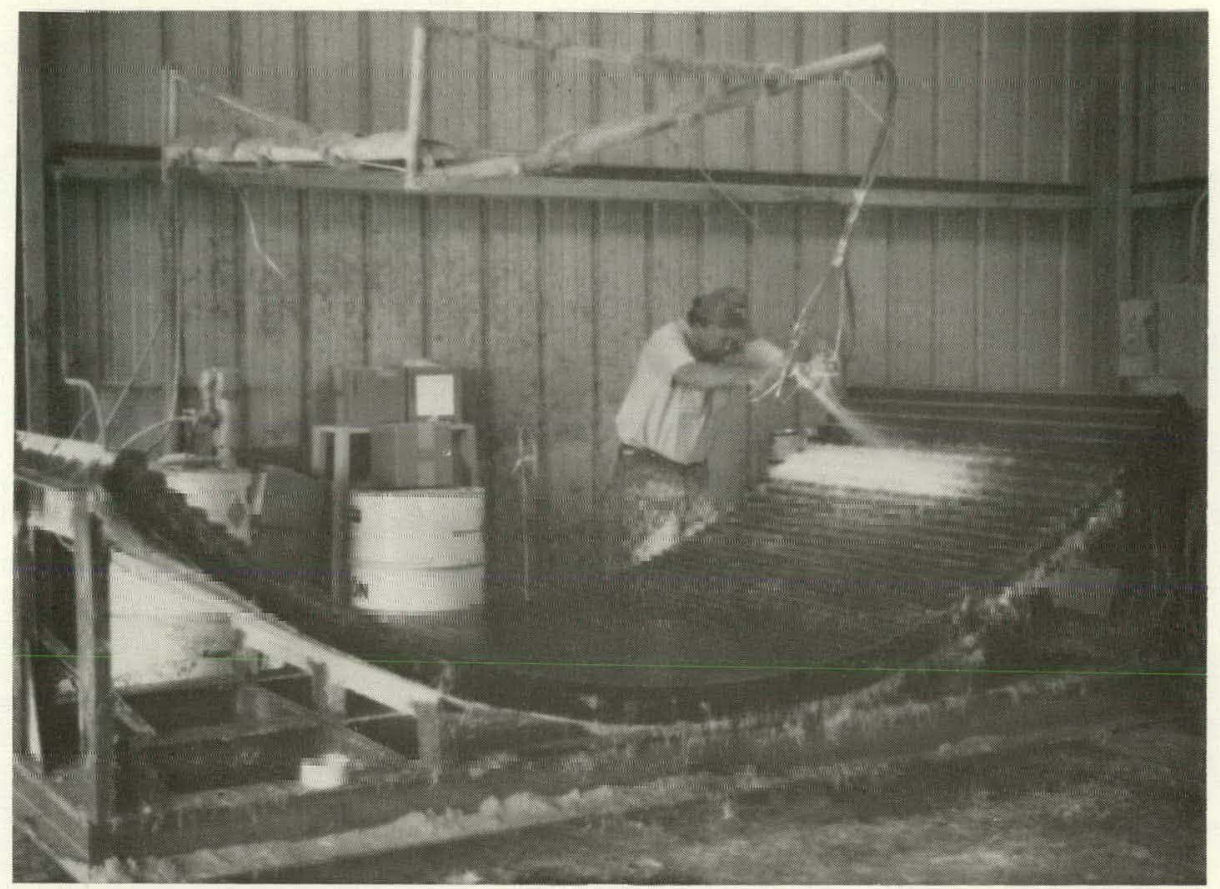

(a)

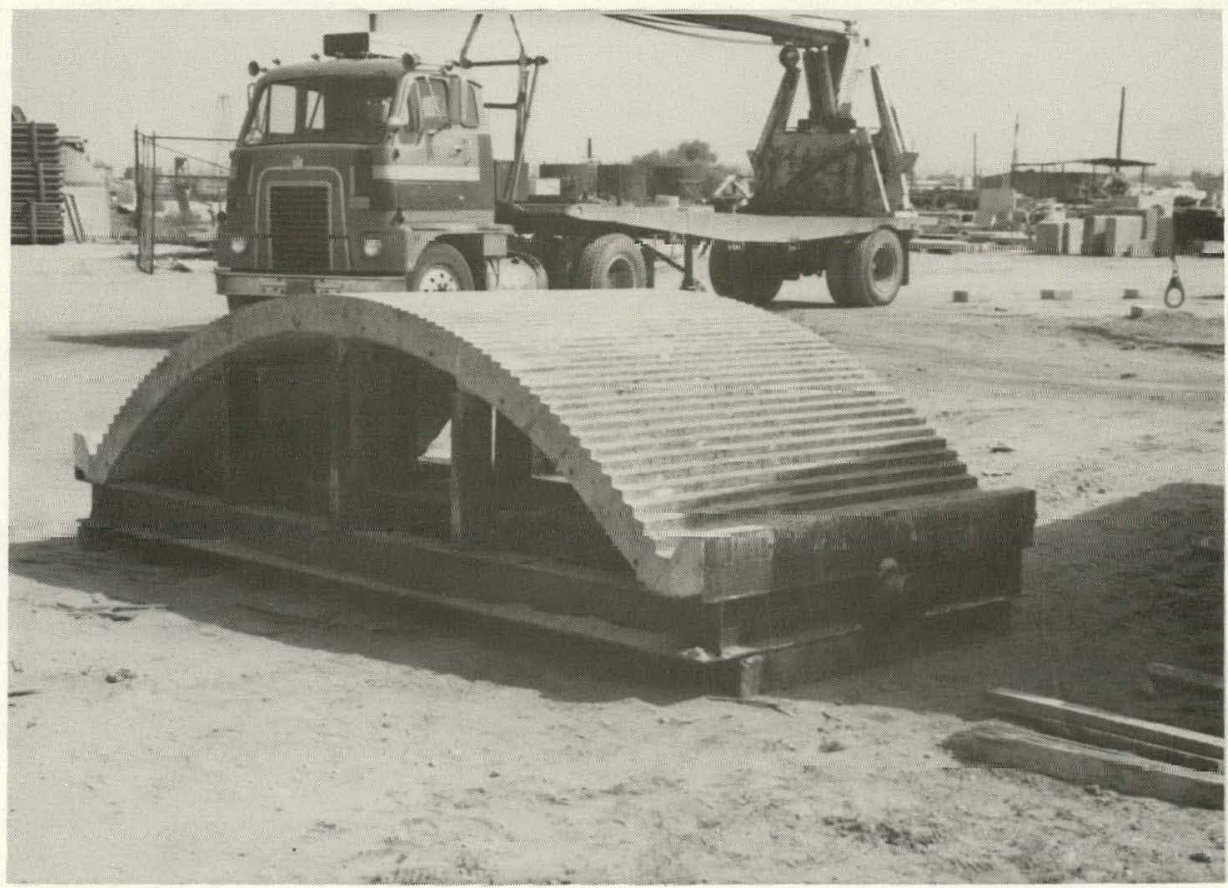

(b)

Fig. 14. Fabrication of fiberglass-coated concrete furm M3: (a) application of fiberglass coat against M2; (b) final product after casting concrete backing 
stick the two forms together. In other areas, the bond between the concrete and the fiberglass was checked by tapping the surface of the form with a pencil and listening to the sound. Except in very few spots, the bond was good. The M3 slat angles were measured after PM3-1 was cast.

Despite the loose edges and the surface imperfections, the slat angles reproduced reasonably well (see Section 4.3.7), the overall appearance of form M3 was good, the form did not distort or crack, and the steel frame appeared to be sufficiently sturdy. Hence, it was concluded that the fiberglass-concrete combination can be used to build the main working forms of the SolarOil project.

The form imperfections will be substantially reduced if the fiberglass is cast upon a smooth, good-quality steel or fiberglass form. The release agent should be applied in thin coats, with much care. During phase II, the edge details should be more thoroughly designed, perhaps with a mechanical connection between the fiberglass and the concrete, and tests should be conducted to determine the reuse factor of this type of form and the effects of form vibrators, steam curing, and stripping.

\subsubsection{Concrete Panels}

The first concentrator panel, PM1-1, was cast against the steel master form M1. The reinforcing was welded wire fabric, supplemented by steel bars at the critical points. The slump was $50 \mathrm{~mm}$ (2 in.), and the concrete was vibrated by a "stinger" type vibrator and hand tapping. The panel was steam cured at $49^{\circ} \mathrm{C}\left(120^{\circ} \mathrm{F}\right)$ for $10 \mathrm{~h}$ and stripped at $72 \mathrm{~h}$ using lifting eyes placed at each corner [Fig. 15(a)]

The panel was easily stripped from the form, but some corners broke in areas where the slat taper was too straight. The panel was not properly vibrated and had many voids which were later patched with Pour Stone. When the panel was set down to remove the turning bar, a hairline crack developed across its center. Measurements taken on this panel indicated that the 


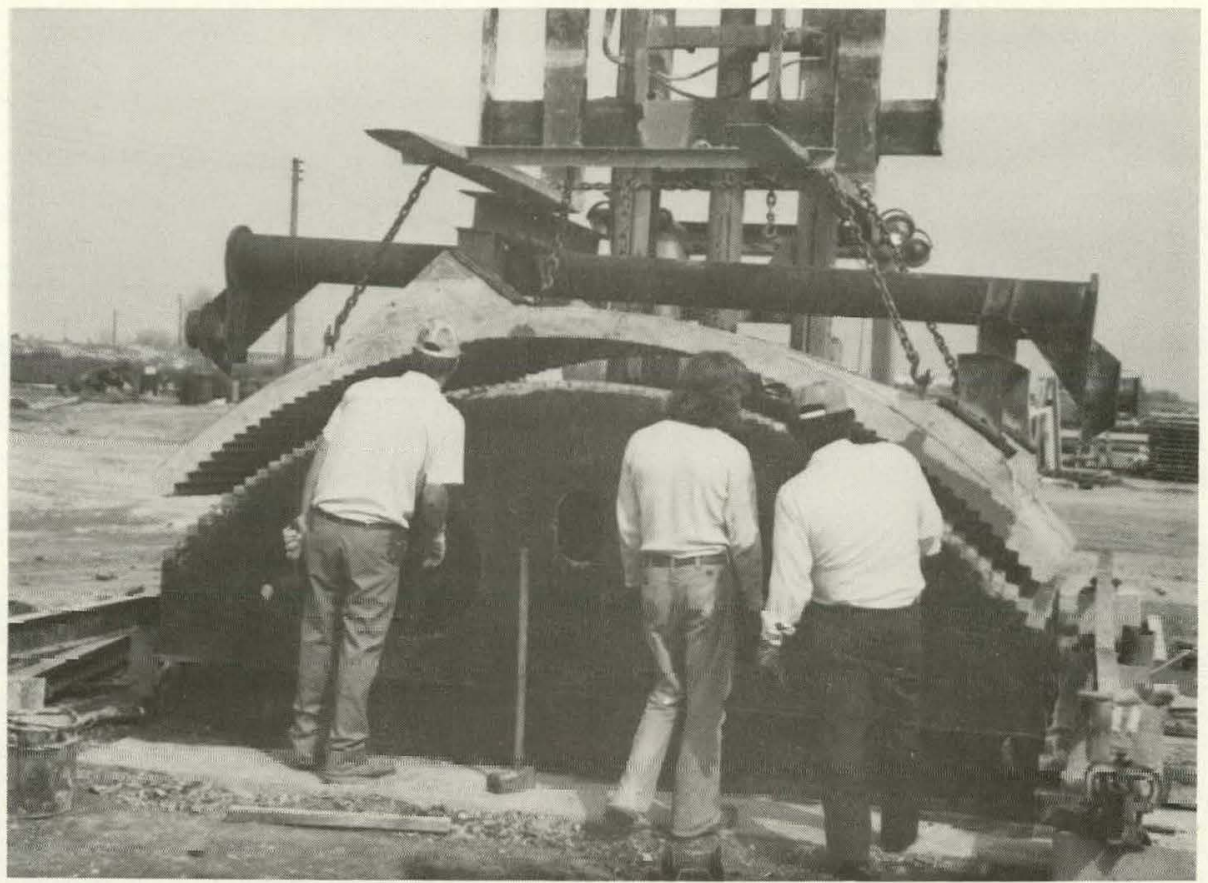

(a)

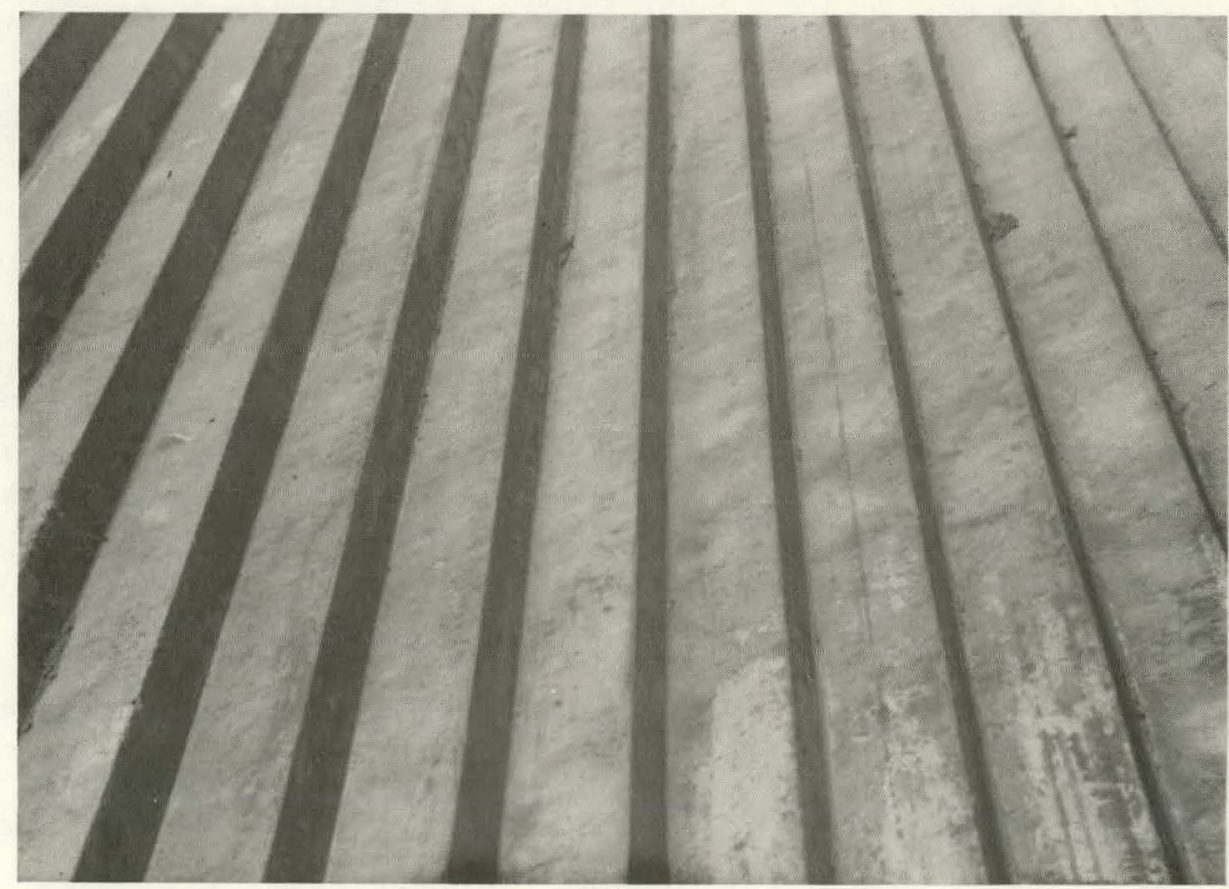

(b)

Fig. 15. Panel fabrication: (a) stripping; (b) close-up of slat surface 
angles were accurately reproduced, even on surfaces with voids. After the voids werc patched, the surface became too irregular to produce meaningful data, and no further readings were taken.

Pane1 PM1-2 was cast against steel form M1 using a concrete mix containing 25-mm (1-in.) long Fibercon steel fibers. Steel bars were placed around the bolt holes and lifting eyes. The slump was increased to $90 \mathrm{~mm}$ ( 3.5 in.), and an extra effort was made to vibrate all the concrete. The panel was steam cured for $12.5 \mathrm{~h}$ at $49^{\circ} \mathrm{C}\left(120^{\circ} \mathrm{F}\right)$ and stripped at $24 \mathrm{~h}$. The surface quality of PM1-2 was better than that of PMI-1 [Fig. 15(b)], but sLIII not up to its full potential. A form vibrator must be used to ensure a good surface. Lack of time made it impossible to test different form vibrators; this will be done in phase II.

Panel PM3-1 was cast against fiberglass/concrete form M3 using standard steel reinforcing bars. A 100-mm (4-in.) slump and stinger vibration were used. The panel was steam cured for $14 \mathrm{~h}$ at temperatures of up to $77^{\circ} \mathrm{C}$ $\left(170^{\circ} \mathrm{F}\right)$ and was stripped at $19 \mathrm{~h}$. The panel developed a hairline crack during the curing period, and its edges lifted slightly off the form. The crack could have been caused by the high steam temperature or a difference in expansion between the form and the pane1. There werc no problems with stripping, but the release agent was affected either by rain during casting or by the excessive curing temperature, leaving the form dirty.

The quality of the finished surface was only fair because of the imperfections in the M3 mold. The surface quality could be improver by taking more time to prepare the $\mathrm{M} 1, \mathrm{M} 2$, and $\mathrm{M} 3$ forms. It is very important that the inilfal mold work be as good as possible.

The panel reinforced with steel fibers seemed to be the strongest of the three. It exhibited no cracks during handling, and the small, sharp corners seemed stronger. Furthermore, the form oil could be applied just prior to placement of the concrete, resulting in easier stripping. With steel bars or mesh, the form oil is applied prior to bar or mesh installation 
to prevent accidental coating of the reinforcement with oil. By the time the concrete is placed, some of the oil drips away and the form surface gets covered with dust, making stripping more difficult. Fiber reinforcement also eliminates the labor required for cutting, bending, and tieing the bars and reduces the possibility of improper placement of the bars in thin slabs.

After stripping, the panels were measured, installed on support posts (Fig. 16), and measured again. Overall, the panels turned out well. They did not warp appreciably, the slat angles reproduced well, and they had goodquality surfaces. The steel fiber reinforcement proved to be the most effective, and the panels were strong enough to be lifted with a forklift without braces or stiffeners. Further testing is needed in phase II to select a proper form vibialui.

\subsubsection{Properties of Concrete}

Table 6 summarizes the properties of the concrete used in the trial mixes and the panels. The trial mixes were used to cast small panels for coating tests (see Section 4.3.1). The concrete was batched and truck-mixed for 100 revolutions. The batch size varied between 3 and $4.3 \mathrm{~m}^{3}$ ( 4 to 6 $\left.\mathrm{yd}^{3}\right)$. Type $\mathrm{V}$ cement was used in trial mix 3 and type II in all other mixes. The maximum aggregate size was $9.5 \mathrm{~mm}$ ( $3 / 8 \mathrm{in.}$ ), except in form $\mathrm{M} 3$, where it was $25.4 \mathrm{~mm}$ ( 1 in.). Steel fibers were used in trial mixes 2 and 3 at the rate of $71 \mathrm{~kg} / \mathrm{m}^{3}\left(120 \mathrm{lb} / \mathrm{yd}^{3}\right)$ and in panel PMl-2 at the rate of $53 \mathrm{~kg} / \mathrm{m}^{3}$ (90 $\left.1 \mathrm{~b} / \mathrm{yd}^{3}\right)$. The fibers mixed well with the concrete and did not cause any problems during casting; however, fiber-reinforced concrete was harder to finish. The slump ranged from 50 to $100 \mathrm{~mm}$ ( 2 to $4 \mathrm{in.).} \mathrm{All} \mathrm{the} \mathrm{concrete} \mathrm{was} \mathrm{con-}$ solidated with stinger vibrators and steam cured. Continuous records of curing temperature were obtained for panels PM1-2 and PM3-1. Panel PM3-1 was unintentionally overheated. The compressive strengths of all the panels and forms were well above the specified strength of $30.9 \mathrm{MPa}$ ( $4500 \mathrm{psi}$ ). The flexural strengths of fiber-reinforced concrete vere around $4.1 \mathrm{MPa}$ (600 psi), somewhat lower than expected, and vere not affected by the fiber content in the range 53 to $71 \mathrm{~kg} / \mathrm{m}^{3}$ (90 to $1201 \mathrm{~b} / \mathrm{yd}^{3}$ ). 


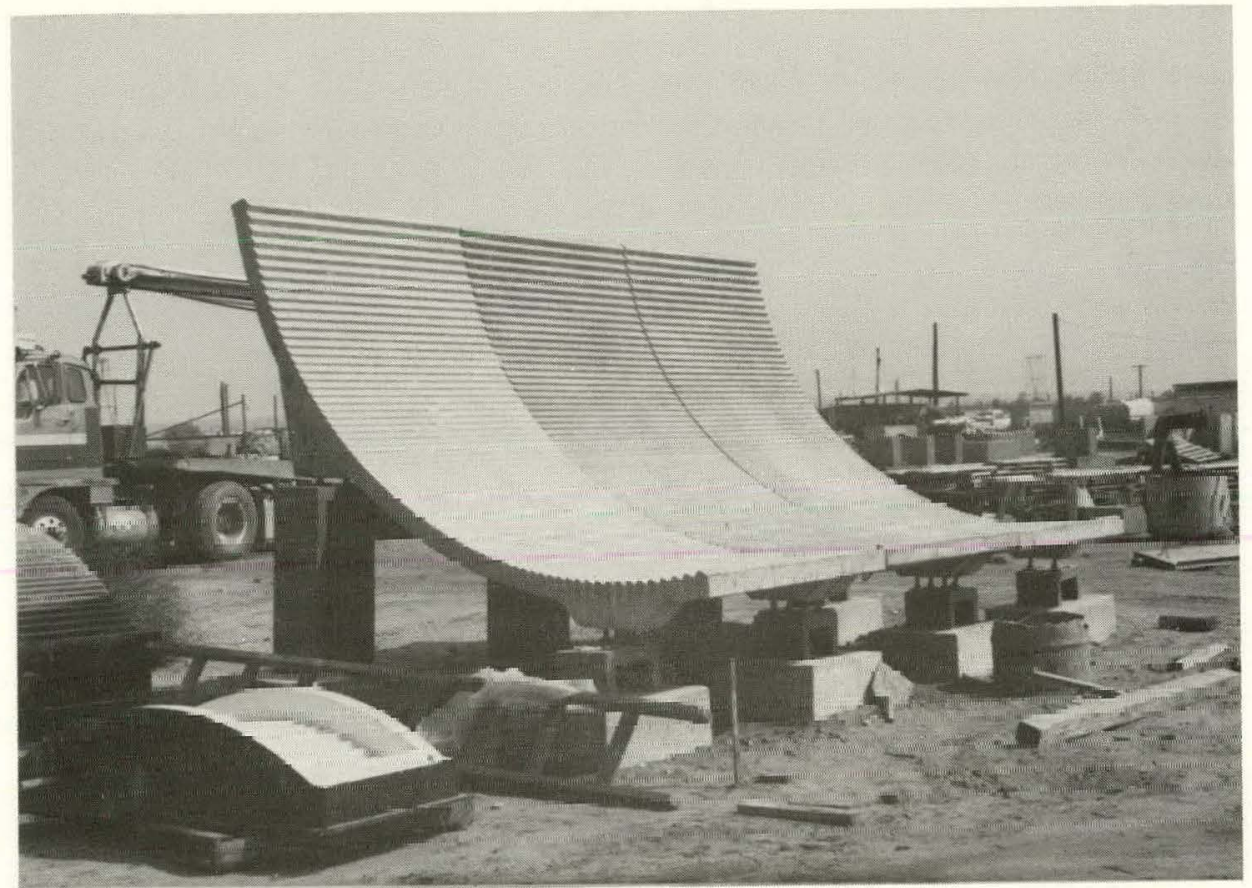

(a)

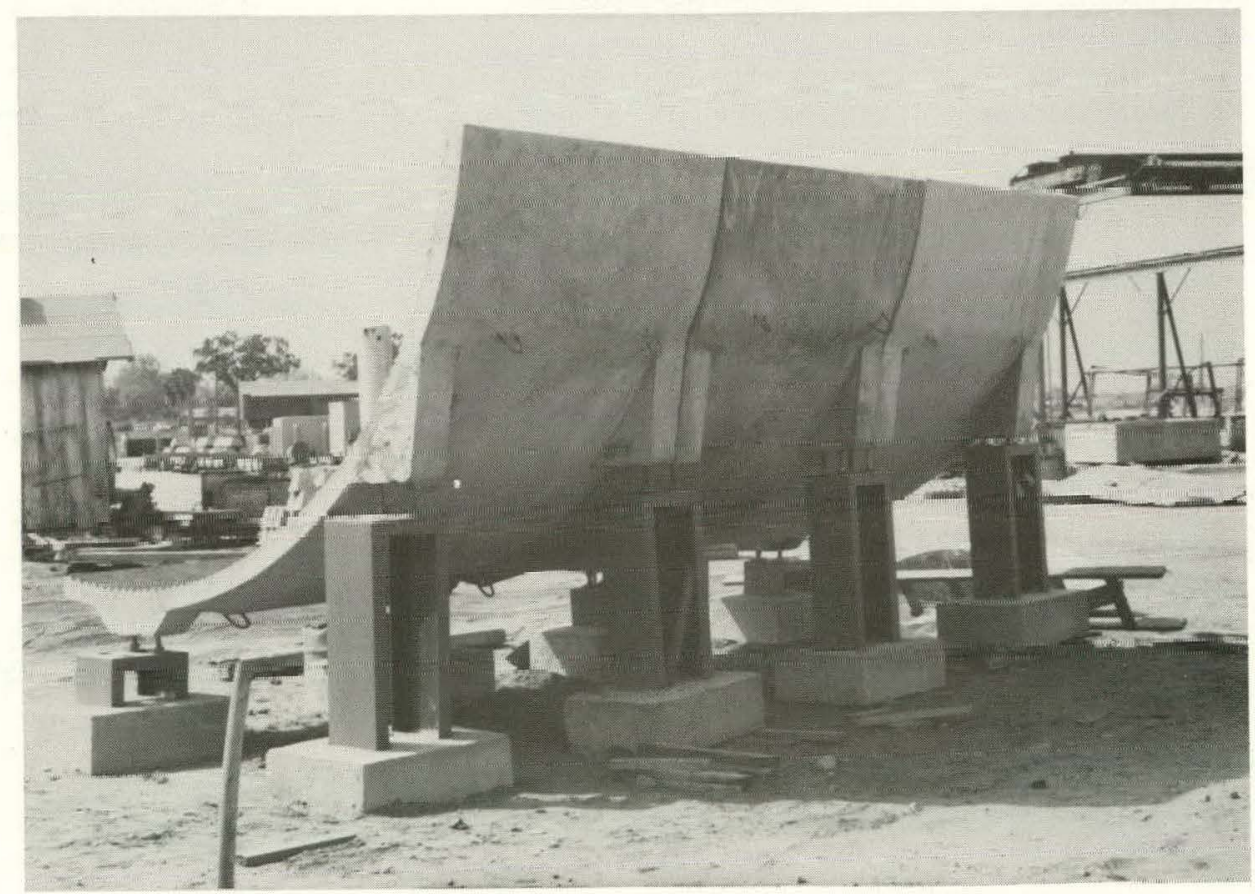

(b)

Fig. 16. Panels in place: (a) front view; (b) rear view 
For best results, future panels should be fabricated with type I, II, or III cement, 9.5-mm (3/8-in.) maximum size aggregate, a 90-mm (3.5-in.) slump, and fiber-reinforced concrete. The concrete should be consolidated with form and stinger vibrators. Fiber reinforcement is a must, since it is the only way to reinforce the delicate slat corners effectively. It also increases the flexural strength of the panels. Steel bars should be used at critical points, and the panels should be steam cured for $12 \mathrm{~h}$ at $49^{\circ} \mathrm{C}\left(120^{\circ} \mathrm{F}\right)$ and stripped the following day. The steam should be applied after the concrete has set. Since panel design is controlled by deflection rather than strength, the specified compressive strength could be reduced somewhat, resulting in a reduction in cement content. The panel thickness should not be reduced. If a more effective steel fiber is found, then the fiber content could be reduced. The steam cusing technique should be antimized and a better parting compound found. The concrete forms should be made with fiberreinforced concrete, since it has superior resistance to impact and vibration.

\subsubsection{Measurement of Slat Angles}

Slat angle measurements were made with a laser inspection fixture developed expressly for this project and described in Section 3.3 of Ref. 1 . The measurements required three setups, as shown in Fig. 17. The first setup was for concave forms and panels, the second for panels installed on supports, and the third for convex forms.

Figure 18 shows how the measurements were made. A mirror was placed on a slat [Fig. 18(c)], a vertical laser beam was aimed at the center of the mirror, and the location of the reflected beam was measured on a target. Then, the slat angle was calculated using trigonometry. For concave forms and panels with properly aligned slats, the laser beam reflections focused at a point in the middle of the target. Incorrectly aligned slats reflected the laser beam to the right or the left of the target center. For panels with a $2.39-\mathrm{m}(7.85-\mathrm{ft}$ ) radius, $25 \mathrm{~mm}$ ( $1 \mathrm{in.}$ ) on the target corresponded to about a $0.15-\mathrm{deg}$ change in angle $\phi$. The laser beam position could be measured to the 


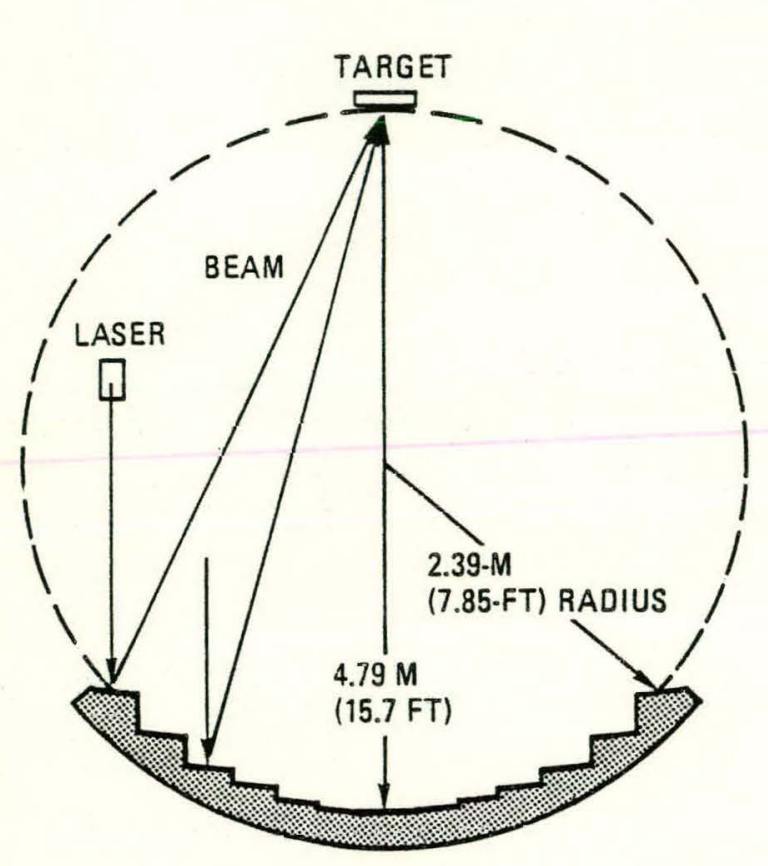

(a)

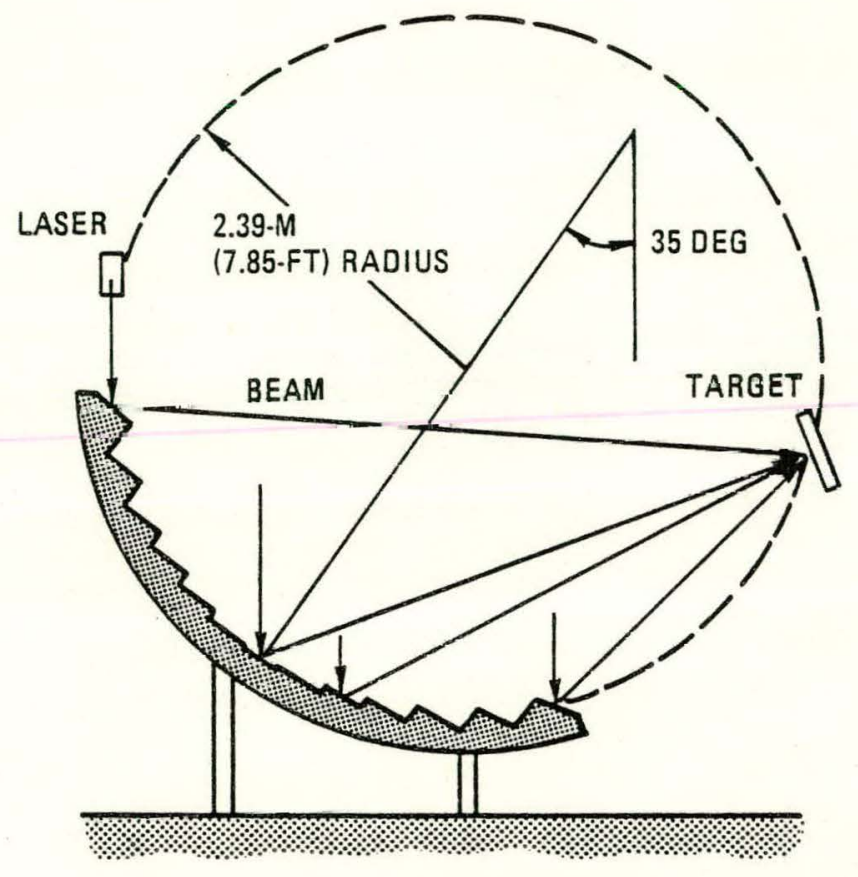

(b)

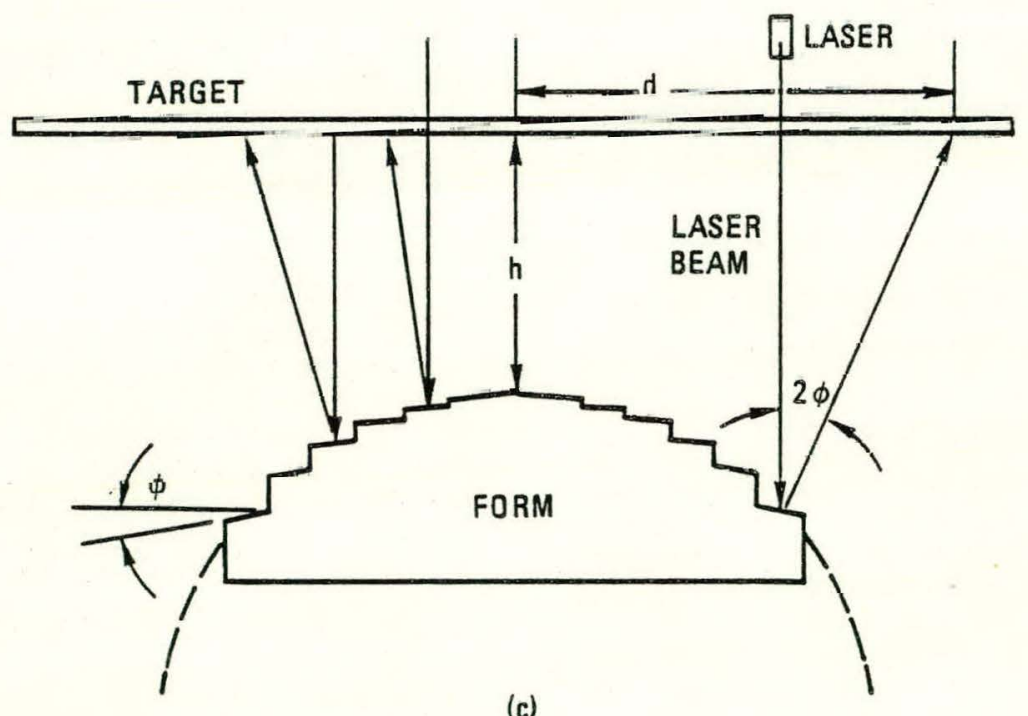

(c)

NOTE: SLAT SIZE EXAGGERATED FOR CLARITY

Fig. 17. Measurement of angle $\phi$ with laser inspection fixture: (a) concave form/ panel; (b) panel on supports; (c) convex form 


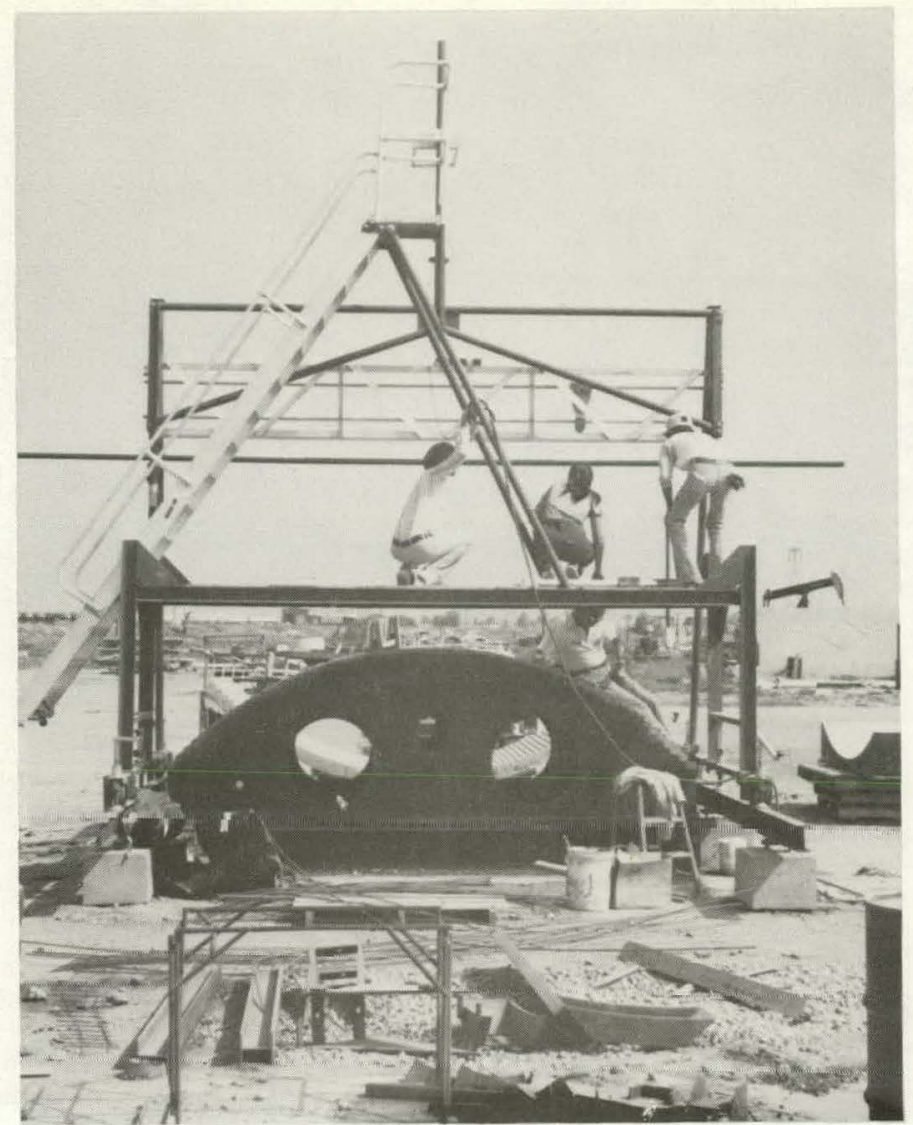

(a)

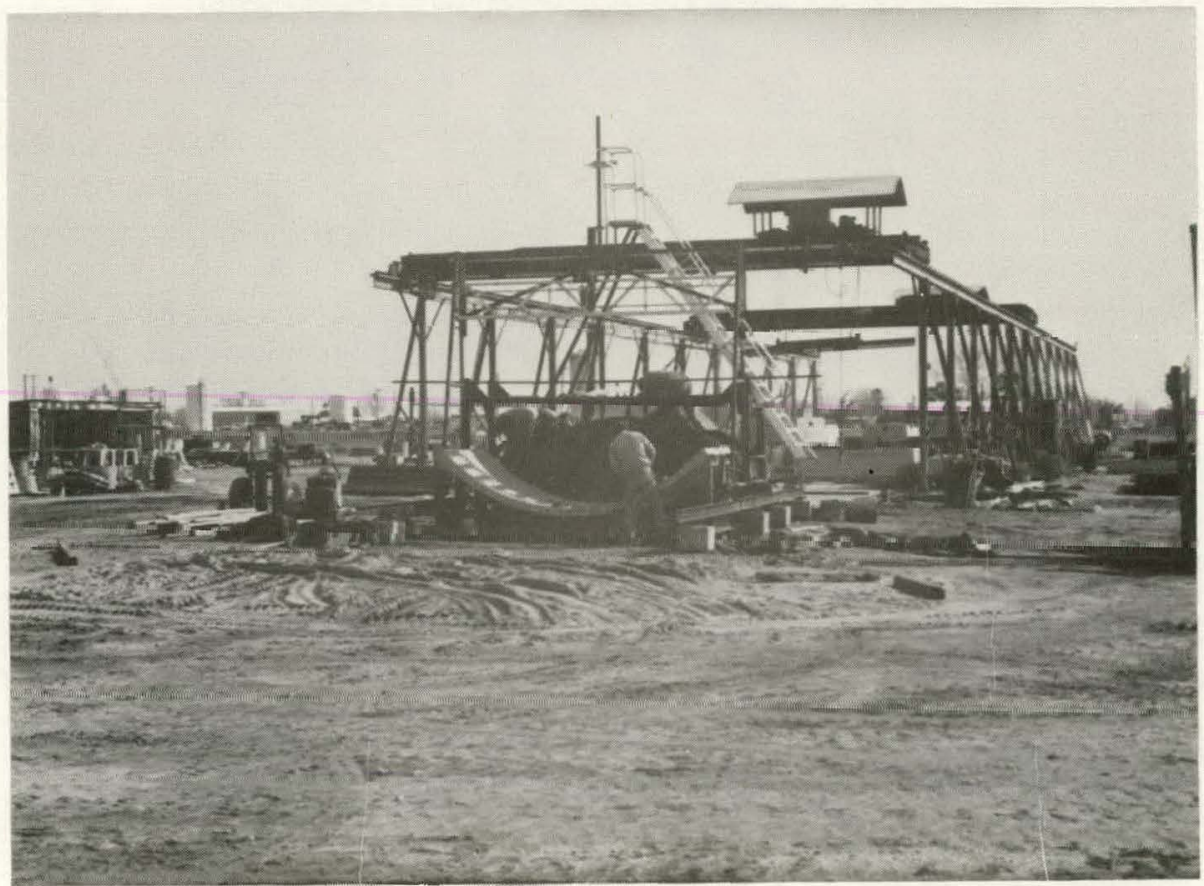

(b)

Fig. 18. Measurement with laser inspection fixture: (a) measurement of convex form M1; (b) measurement of concave form M2 


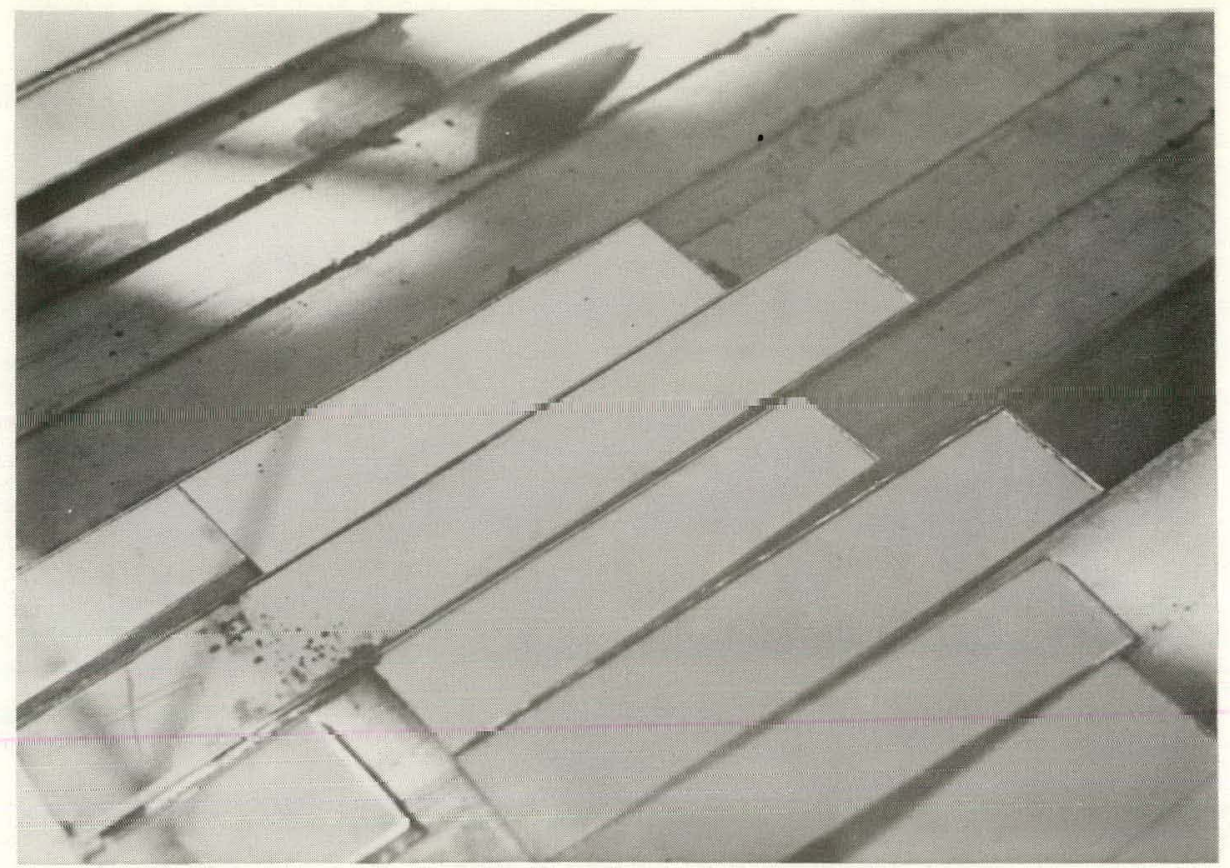

(c)

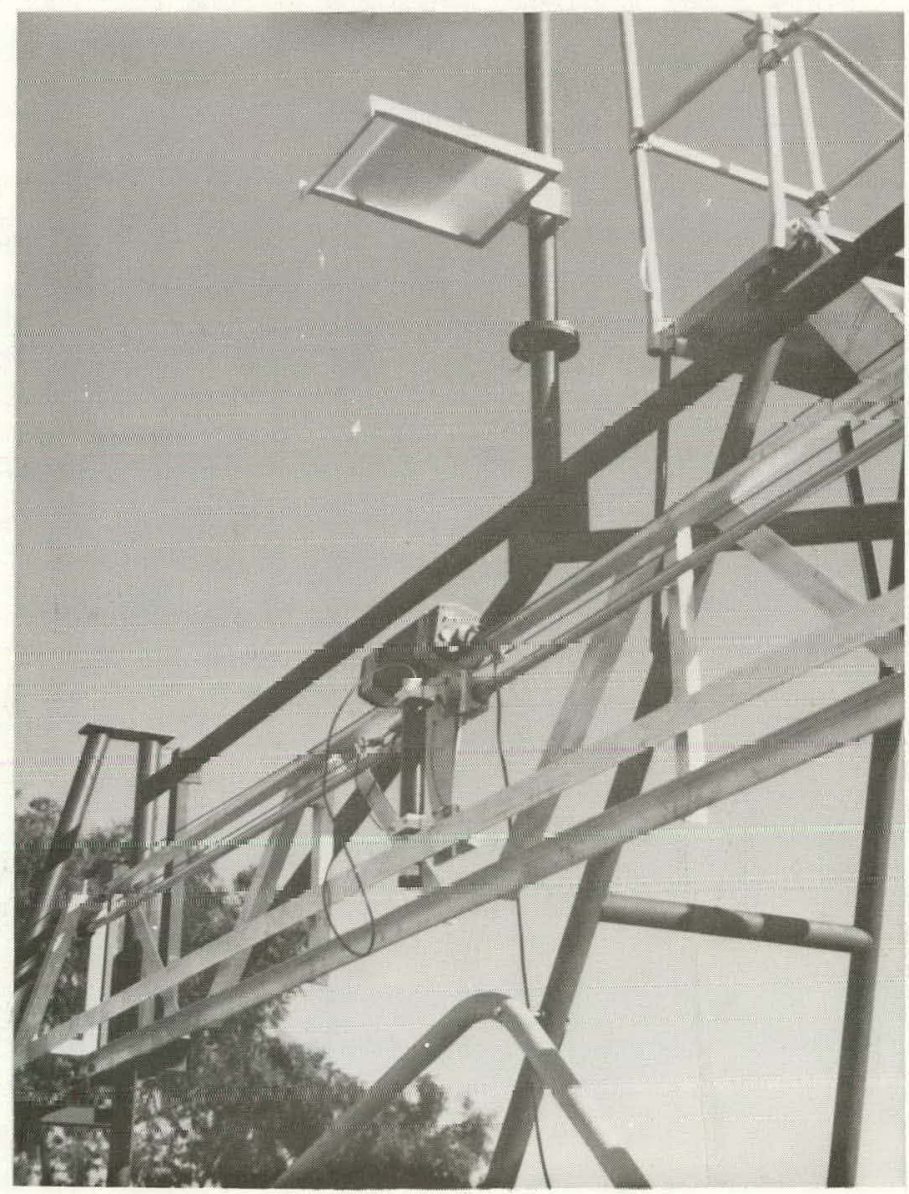

(d)

Fig. 18. Measurement with laser inspection fixture: (c) mirrors on concave concrete surface; (d) laser and target for concave surface measurements 
nearest $0.5 \mathrm{~cm}(0.2 \mathrm{in.})$, which corresponds to a precision of about $0.03 \mathrm{deg}$. This type of measurement was easy to make, and errors of slight laser fixture misalignment were not significant.

Setup 2 was for panels sitting on supports. The principle of measurement was the same as that for setup 1 , except that the target was positioned to the side. The measurements in this position were much more difficult to take than anticipated, because small deviations in panel or laser fixture alignment introduced large errors in slat angle readings.

Setup 3 was for convex forms such as $M 1$ and M3. The laser beams were divergent, and the slat angle accuracy was determined by measuring distance $d$ on the target and comparing it with the theoretical distance calculated from form geometry and measured value of height $h$. Accurate readings were harder to take in this position than in that for setup 1, but easier than in that for setup 2. Care had to be taken to align the laser inspection fixture properly. A certain amount of data reduction was needed to determine whether a slat was properly aligned.

During slat angle measurement with the laser inspection fixture, it is very important that the panel (or form) be level, the fixture aligned, the laser beam vertical, the slat surface clean, and the mirror flat. New mirrors had an uneven back surface and would not lie flat on the slat. A broken-in mirror which had been rubbed against the concrete worked best. The standard procedure was to align the panel (or form) and the fixture, check the verticality of the laser beam and the position of target, remove grit from the slat, lay a broken-in 50 by $100 \mathrm{~mm}$ ( 2 by 4 in.) mirror strip on the slat without applying any pressure to the mirror, and move the laser beam so Lhal it hit the center of the mirror.

All readings on the concrete panels and forms and some of the readings on steel form $M 1$ were taken twice. At the beginning, when the inspectors were learning how to use the fixture, the readings were within 0.08 deg of each other. Toward the end of the program, the readings were within 0.03 deg 
of each other. There were other sources of random and systematic errors associated with the measurement and calculation of the slat angles. These sources and the sensitivity of the slat angles to the errors should be investigated during phase II. Overall, the laser fixture worked well, especially for setups 1 and 3 . However, the fixture will require modification during phase II to make it more stable and easier to use.

Figure 19 and Table 7 indicate the locations of the points at which the slat angle measurements were taken. The forms and panels were marked to maintain slat traceability. One end of form Ml was designated side $A$ and the other side B. When facing side A, slat 1 on form M1 was on the left and slat 71 was on the right. Position 1 was taken approximately $150 \mathrm{~mm}$ ( 6 in.) from side A, position 2 near the center of the form, and position 3, $150 \mathrm{~mm}$ (6 in.) from side B. Form M2 and the panels were the mirror image of M1. Thus, when facing their side A, slat 1 was on the right and slat 2 on the left.

Since all the forms were symmetrical, it was very easy to confuse sides $A$ and B. Form M3 was not properly marked and was turned 180 deg when panel PM3-1 was cast. Hence, the position and slat numbers of panel PM3-1 are 180 deg out of phase with those of panels PMI-1 and PM1-2.

Data determined to be invalid were not included in the analysis, but no reading was arbitrarily excluded simply because it was too high or too low. Readings taken during inspection 3 were believed to be invalid due to wisalignment of the inspection fixture and high wind and werc not reported. Readings taken during inspections 7 through 10 with the panels on supports could not be evaluated because of insufficient data. The initial readings looked unreasonable, and 1 was suspected that the panels and laser fixture were out of alignment. Before the alignment could be rechecked, the supports settled during a rainstorm, and the initial reference point was lost. The loss of these data had no significant effect on the test program, since measurements taken with a steel tape across the panel aperture indicated no significant warpage or deformation. 

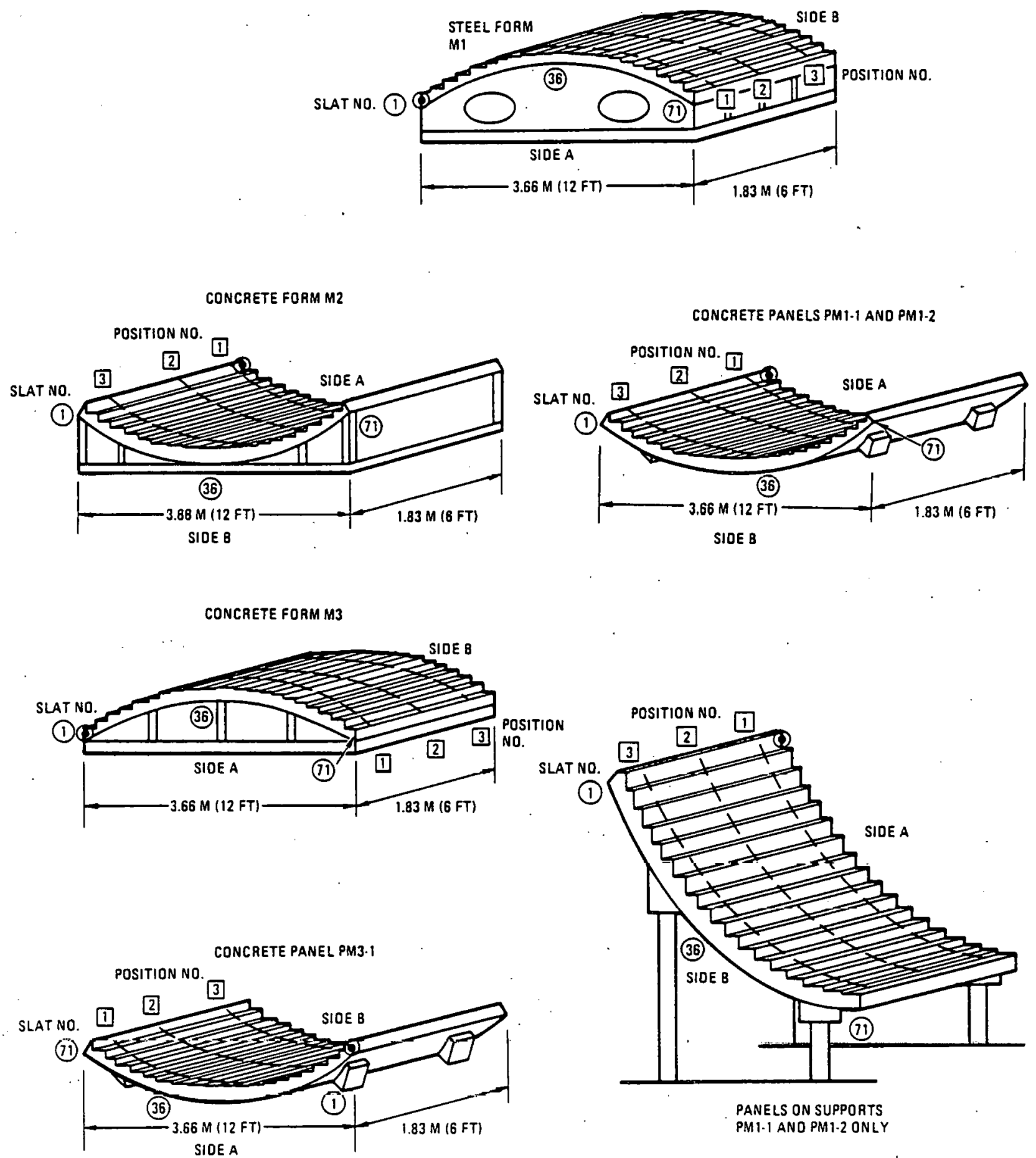

PANELS ON SUPPORTS PMI-1 ANO PM1-2 ONLY

(-) corresponding slat

- Fig. 19. Key to readings taken with laser inspection fixture 
The data collected in the field were reduced using computer programs written expressly for this project. Appendix A compares the achieved and desired slat angles, and Table 8 shows the number of valid readings and the mean and the standard deviation $\sigma$ of the differences between the achieved and the desired slat angles. With the standard normal distribution curve commonly used to evaluate statistical data, $68.27 \%$ of the results fall within $\pm 2 \sigma$ from the average, $95.45 \%$ fall within $+2 \sigma$ from the average, and $99.75 \%$ fall within $+3 \sigma$ from the average. The $2 \sigma$ and $3 \sigma$ values for slat angle differences were of the order of 0.25 and $0.39 \mathrm{deg}$, respectively. This was believed to be quite good, considering it was a first-of-a-kind operation. With proper care, the slats on form $M 1$ can be shimmed to bring the $2 \sigma$ value to within $+0.07 \mathrm{deg}$ and the $3 \sigma$ value to within $+0.10 \mathrm{deg}$ of the desired angle. This will in turn improve all the descendants of MI.

The fiberglass-coated concrete form $\mathrm{N} 3$ had loose fiberglass around its periphery and had inherited small surface bumps from the voids in M2. Consequently, its $3 \sigma$ was $\pm 0.60 \mathrm{deg}$, which is not a very good value. This value can be improved by casting M3 against a smooth steel or fiberglass surface and carefully gluing the fiberglass coating.

Appendix $B$ compares the data on the slat angles of the panels and forms; Table 9 summarizes these data. The $3 \sigma$ values ranged from 0.20 to $0.66 \mathrm{deg}$, with the lowest values representing the differences between the panels and forms cast from the same mold. The slat angle data imply that the panels opened somewhat. However, the measurements taken with a steel tape across the aperture indicated only a 1.5-m. (0.06-in.) increase in PM1-2 and no increase in M2, PM1-1, or PM3-1. The tape measurement was considered more reliable, confirming that the panels did not warp appreciably.

Overall, the results are very encouraging. It is likely that the slat angle data given in Tables 8 and 9 will be improved after corrections are made for measurement errors. 


\subsubsection{Panel Supports and Installation}

Figure 16 shows the panel supports used during the test program. For economic reasons, the supports were made with steel channels rather than the steel pipe considered for the prototype. The concrete footings were precast and positioned on uncompacted natural grade, and the panels were installed using a forklift with two slings wrapped around the panel near the support humps. A $10-$ to $20-\mathrm{mm}(0.4-$ to $0.8-\mathrm{in.})$ gap was left between the panels, and the panels were aligned with leveling nuts. After the panels were installed, the supports settled somewhat, especially after the first rain. This affected the slat angle readings taken with the laser inspection fixture.

No problems are foreseen in the design of the supports, which will be done during phase II using pipe or channel. The foundation will be designed to minimize differential settlement. Use of a larger single bolt rather than two smaller bolts at each panel support point might improve the connection between the panel and support post. This will be investigated in phase II.

\subsection{CONCLUSIONS AND RECOMMENDATIONS}

\subsubsection{Conclusions}

The following has been accomplished:

1. A $1.83-\mathrm{m}(6-\mathrm{ft})$ long steel form was built, with $95.45 \%$ of the slat surfaces within $+0.18 \mathrm{deg}$ of the desired value and $99.75 \%$ within $\pm 0.28 \mathrm{deg}$. Six slats were shimmed to demonstrate that the form can be adjusted to have $99.75 \%$ of its surface within to.10 deg of the desired value. The form did not distort after several castings and was judged to be good for several hundred reuses without major returbishing.

2. Two concrete forms were fabricated. The first was cast against the steel form; the second had a fiberglass surface and was cast 
against the first concrete form. The ruggedness and overall appearance of both forms were good. However, the concrete form without fiberglass had too many surface voids to be used as production form. The fiberglass-coated form had a smooth surface except in areas where the concrete form from which it was cast had voids. These defects can be eliminated by casting the fiberglass against a smooth steel or fiberglass surface. This type of form was judged to be good for 35 to 50 reuses without major refurbishing and to be suitable for fabrication of production panels.

3. Twn rnnerete panels wero caot from the steel fulu dnd one from the fiberglass-coated concrete form. All panels were $1.83 \mathrm{~m}$ ( $6 \mathrm{ft}$ ) long, $114 \mathrm{~mm}$ ( $4.5 \mathrm{in.}$ ) thick, and $3.6 \mathrm{~m} \mathrm{(11} \mathrm{ft} 10 \mathrm{in.}$ ) wide. The best panel was reinforced with steel fibers and cast from the steel form. It had $99.75 \%$ of its surface within \pm 0.29 deg of the desired angle. This panel had good surfaces and rugged corners and did not warp appreciably. The panel cast from the fiberglass-coated concrete form had somewhat inferior surfaces and slat angle accuracy, mainly because of surface deferts in the form from which it was casl. Structural calculations showed that the panel length can be increased to $5.5 \mathrm{~m}$ (18 ft) without appresiahly affecting the accuracy of the slat angles.

4. The proper concrete mixes, parting compounds, placement methods, vibrating techniques, and curing procedures were determined, and hardware and techniques for stripping and turning the panels were rested. A laser inspection fixture that can accurately measure slat angles was developed and tested. Areas of improvement were identifled and recommendations to achleve these improvements made.

5. Based upon the test results and structural calculations, it is concluded that reasonably priced $5.5-\mathrm{m}(18-\mathrm{ft})$ long panels can be produced with either steel or fiberglass-coated concrete forms with $95 \%$ to $99 \%$ of the slat area within +0.25 deg of the desired angles. 
With steam curing, the production rate. will be one panel every other working day per $5.5 \mathrm{~m}(18 \mathrm{ft}$ ) of form length.

\subsubsection{Recommendations for Fabrication of Panels and Forms}

Based on the results of this program, the following recommendations are made :

1. The panel length should be $5.5 \mathrm{~m}(18 \mathrm{ft}$ ) and the thickness $114 \mathrm{~mm}$ (4.5 in.).

2. The panels should be cast every other day and should be made of fiber-reinforced concrete. The specified compressive strength of the concrete should be $31.0 \mathrm{MPa}$ ( $4500 \mathrm{psi}$ ), the maximum aggregate size $9.5 \mathrm{~mm}$ ( $3 / 8 \mathrm{in.})$, and the slump $90 \mathrm{~mm}$ ( $3.5 \mathrm{in.})$. Steel reinforcing bars should be provided at critical points, and the fiber content should be $53 \mathrm{~kg} / \mathrm{m}^{3}\left(90 \mathrm{lb} / \mathrm{yd}^{3}\right)$. Lightweight concrete is not cost effective.

3. The concrete should be consolidated with stinger and form vibrators.

4. Steam curing should be at $49^{\circ} \mathrm{C}\left(120^{\circ} \mathrm{F}\right)$ for $12 \mathrm{~h}$, starting after the conerete has oct (about $1.5 \mathrm{~h}$ after casting).

5. The panels should be washed immediately after stripping.

6. The forms can be made either of steel or fiberglass-coated concrete. Steel forms are durable but expensive, require a long lead liule for fabrication, and arc sueceptible to corrosion. Fiberglass-coated concrete forms of good quality can be economically and rapidly fabricated once a good master steel form is produced. These forms are not susceptible to corrosion, but their 
durability is inferior to that of steel. The final form selection should be based on cost per panel and delivery schedule.

7. To increase the accuracy of fiberglass-coated concrete forms, the following should be investigated: elimination of $M 1$, manufacture of several convex M2 steel forms, and casting of fiberglass-coated concrete forms from steel form M2.

8. Concrete forms should be reinforced with steel fibers for better resistance to vibration and impact loads.

9. The draft of the slat angles should be increased to $1.0 \pm 0.25$ deg.

10. All steel forms should be assembled and checked for alignment at the place of fabrication. All surface defects must be repaired.

11. The backs of the mirror surfaces should be flat and the mirrors snlidly attached to the cunciele.

12. Panels and forms should be inspected with the laser fixture and their apertures measured with steel tape. Once the quality of the panels is established, the number of inspections can be substantially redured.

13. Papid installatiul and alignment should be accomplished by prealigning the support posts and adjusting the panel angle with leveling nuts.

14. There should be only one anchor bolt at each panel support point. 


\subsubsection{Recommendations for Phase II Tests}

During phase II, at least two 5.5-m (18-ft) long panels should be fabricated from the existing $M 1$ steel form and measured to confirm their suitability. Tests should be conducted to determine the reuse factor of the fiberglass-coated concrete forms, with emphasis on the effects of form vibration, steam curing, and stripping. In addition, the following should be done to improve panel quality and reduce cost:

1. The edge details of forms M1, M2, and M3 should be designed and the benefits of prestress in panels assessed to facilitate stripping.

2. The panel supports should be designed with on $\perp y$ one bolt per panel at each support.

3. The panel lifting points should be designed.

4. The specified compressive strength of the concrete should be reassessed. This could allow a reduction in the cement content.

5. A more effective fiber reinforcement should be found so that the fiber content can be reduced.

6. The concrete mix should be optimized.

7. The steam curing and stripping procedures should be optimized.

8. A parting compound that works well with steam curing, steel forms, and fiberglass furms sliould be found.

9. The available fiberglass product should be examined, and the one that best suits our needs selected. 
10. Form vibrators should be studied.

11. Sources of error in measuring slat angles should be investigated, especially with panels on supports.

12. Minor modifications should be made to the laser inspection fixture so that it is more accurate and easier to use.

13. The possibility of eliminating form M1 and making concave form M2 of steel should be investigated.

14. Methods of gluing the mirrors to the slats should be investigated. 


\section{ACKNOWLEDGMENTS}

We would like to acknowledge the guidance and support provided by J. M. Neville, the Solaroil Program Coordinator at the Department of Energy San Francisco Operations Office, and E. J. Rattin, the Program Coordinator from Aerospace Corporation, which served as the technical monitor for DOE. We would also like to thank the principal subcontractors who participated in the panel development program: J. Herndon, Chief Engineer, Hopper Incorporated; J. Misemer, President, Bakersfield Precast Company; R. Morales, Plant Superintendent, Bakersfield Precast Company; I. Garza, Yard Foreman, Bakersfield Precast Company; and D. Dimaggio, President, Mr. Fiberglass Company .

The major GA contributors to the concrete concentrator panel development program were F. Dahms; J. Neill; G. Chow; V. Nicolayeff; M. Koploy; D. Ryder; G. Connors; and L. Brenn. 


\section{REFERENCES}

1. Baccaglini, G., et al., "SolarOil Project, Phase I Preliminary Design Report," DOE Report GA-A15823, General Atomic Company, March 1980.

2. Johnson, J. J., MODSAP, A Modified Version of the Structural Analysis Program SAP IV for the Static and Dynamic Response of Linear and Loçalized Nonlinear Structures," DOE Report GA-Al4006 (REV), General Atomic Company, June 1978. 
APPENDIX A

SLAT ANGLE MEASUREMENT DATA

\begin{tabular}{c}
$\begin{array}{c}\text { Inspection } \\
\text { No. }\end{array}$ \\
\hline 1 \\
2 \\
4 \\
5 \\
6 \\
11 \\
12 \\
13
\end{tabular}




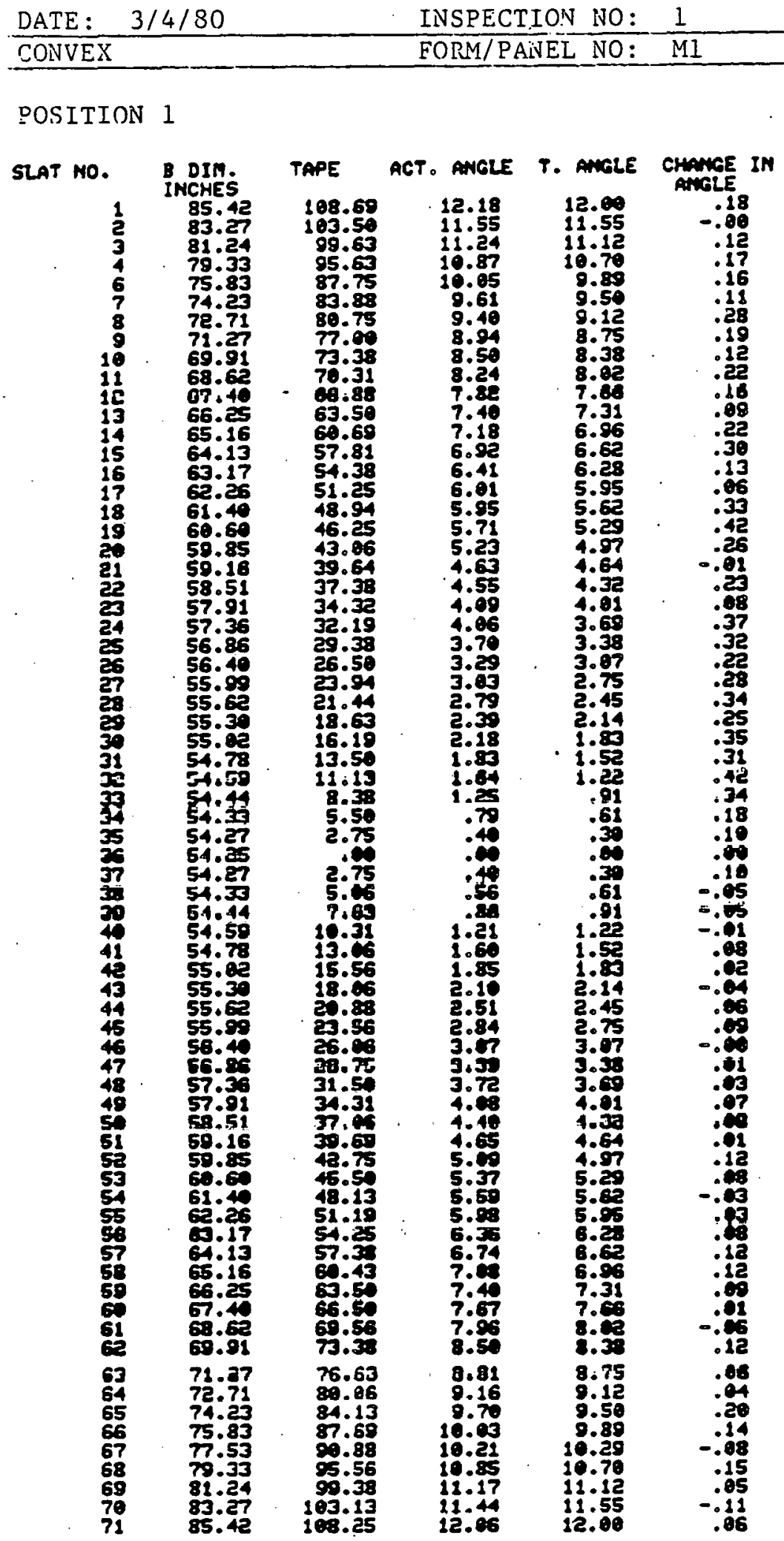




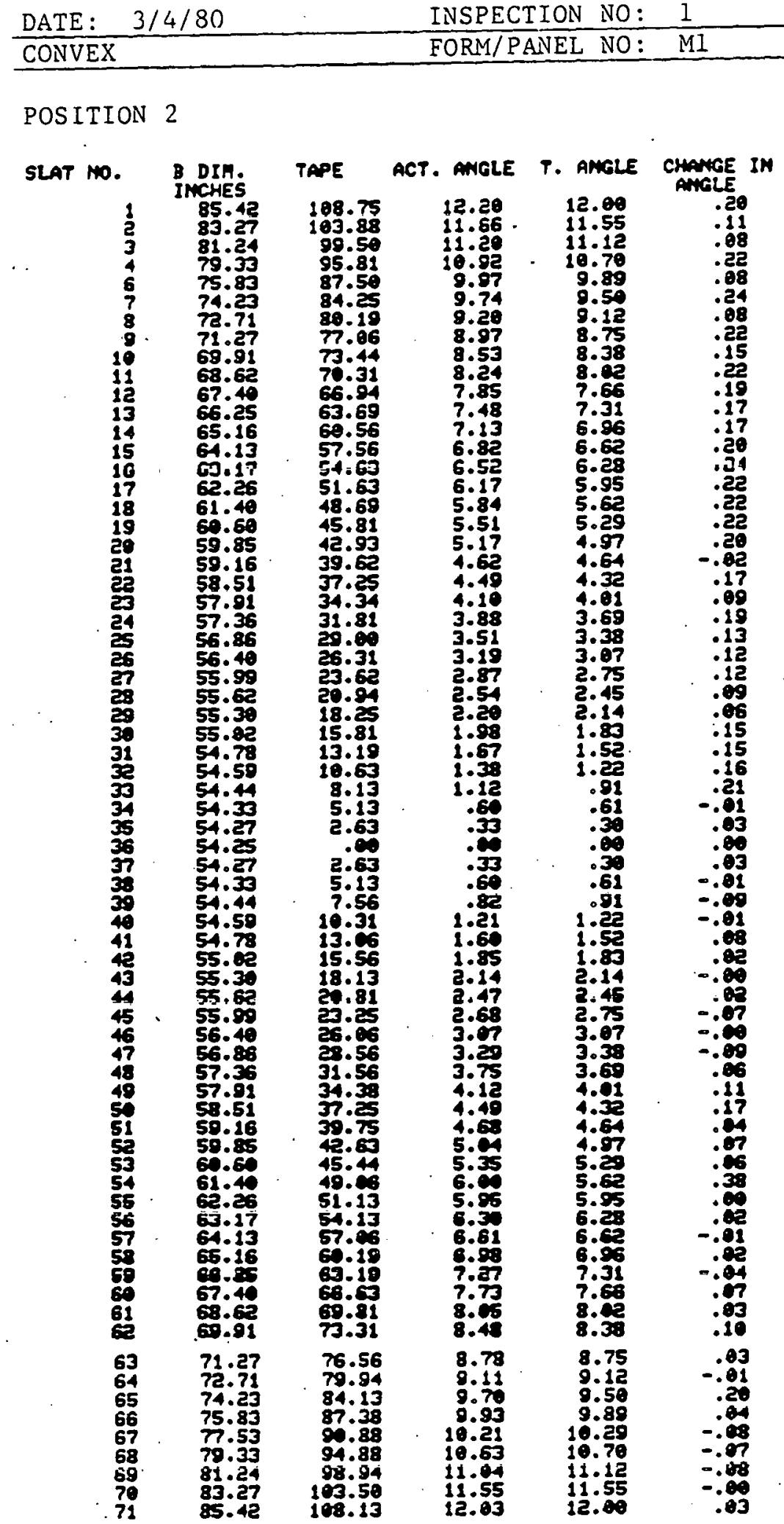




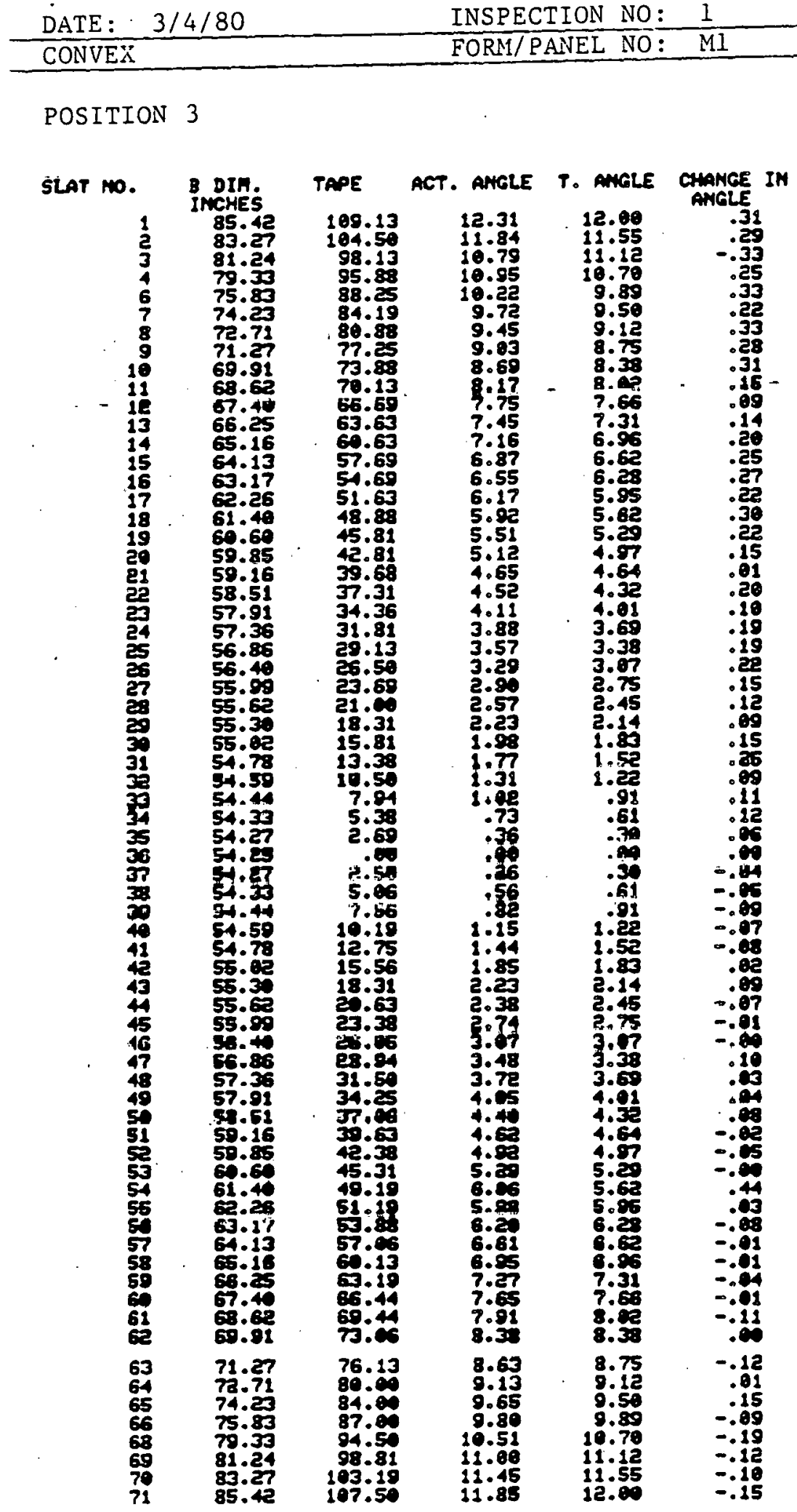




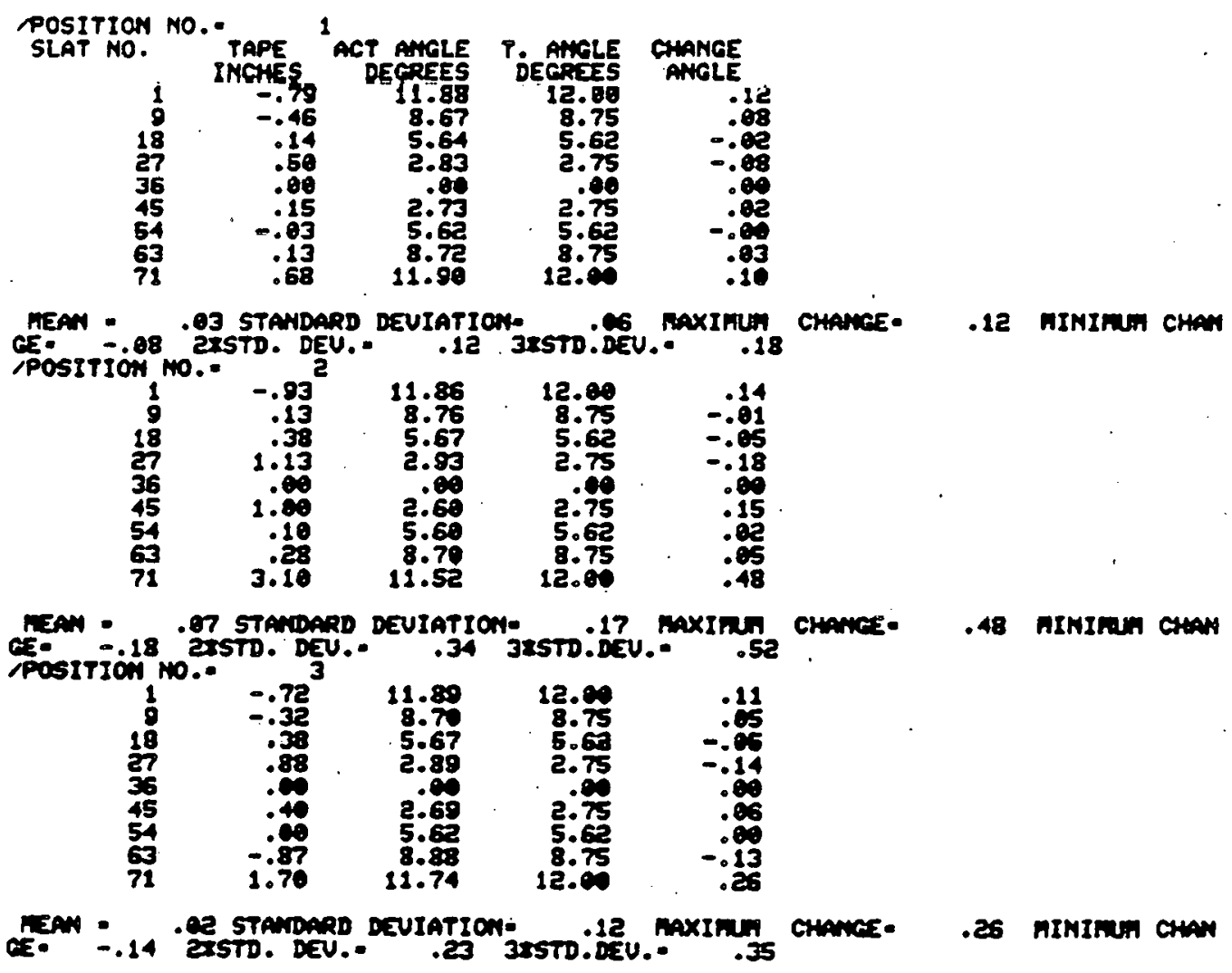




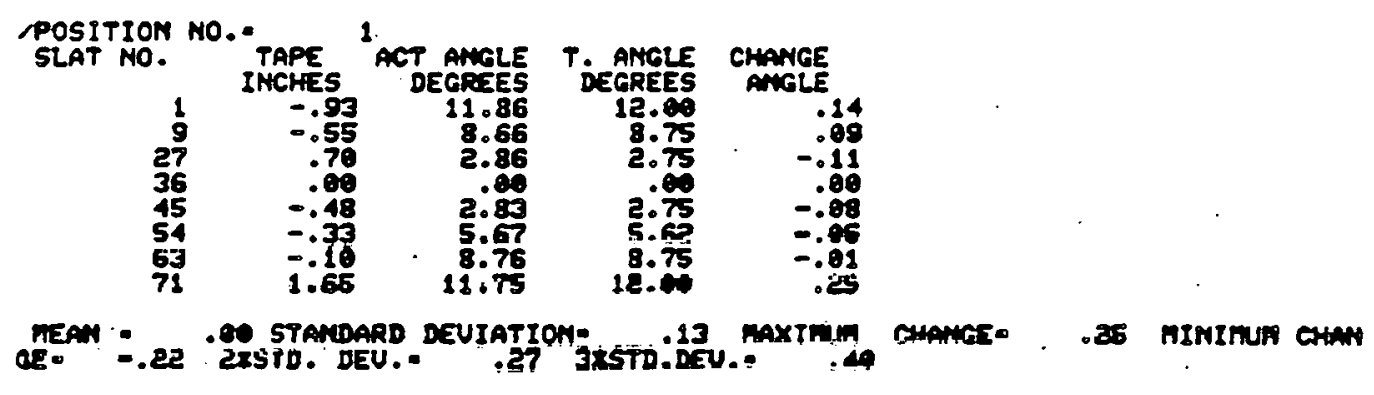




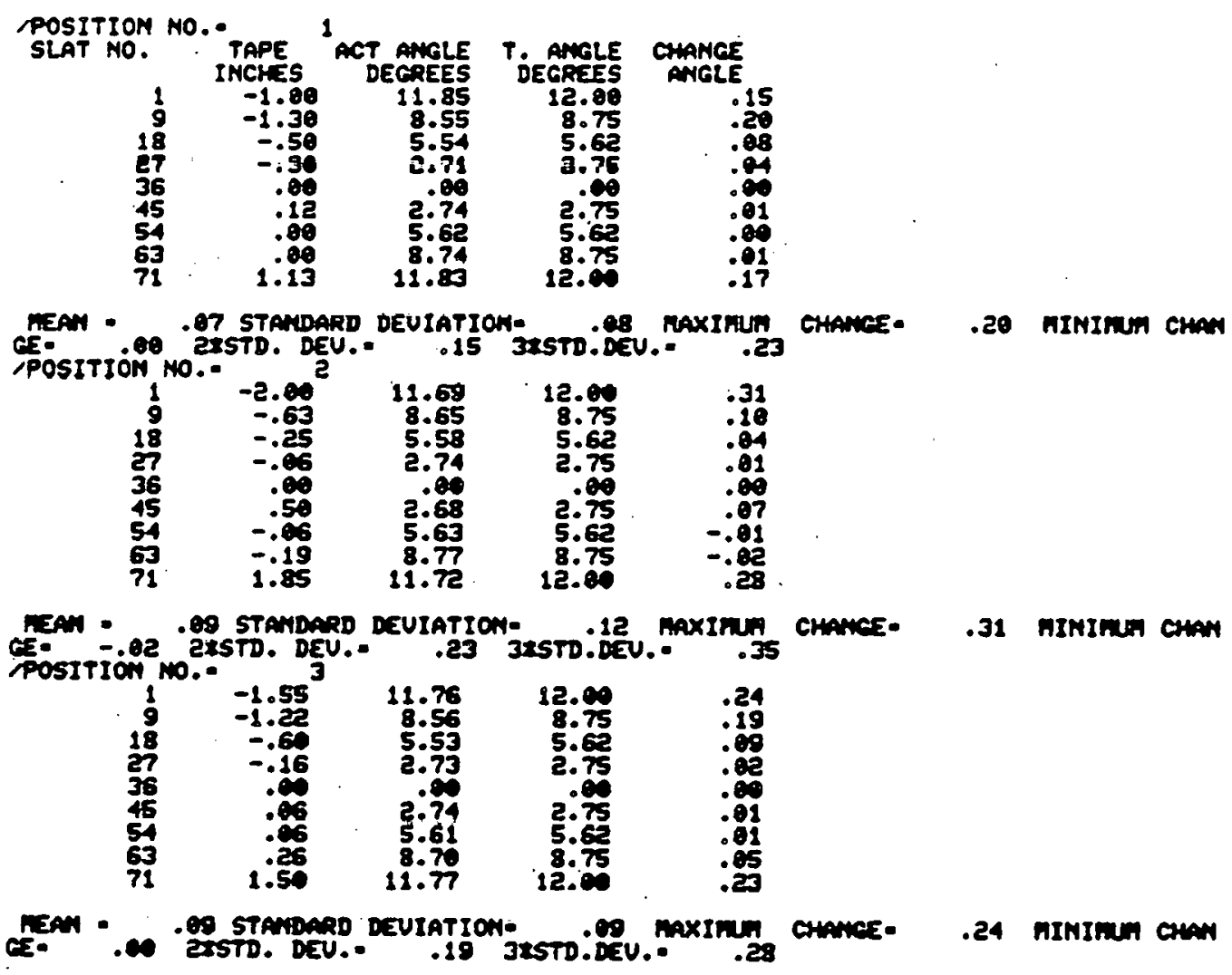


DATE: $\quad 3 / 20 / 80$

INSPECTION NO: 6

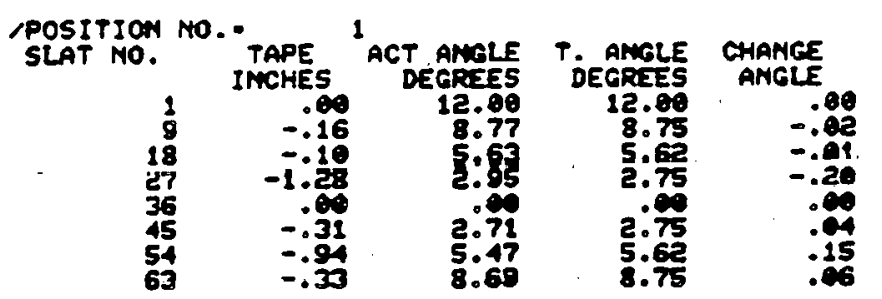

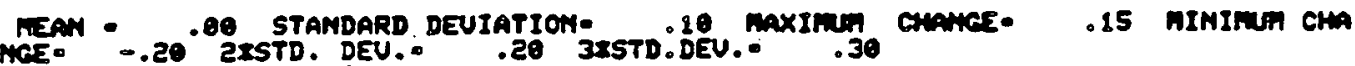

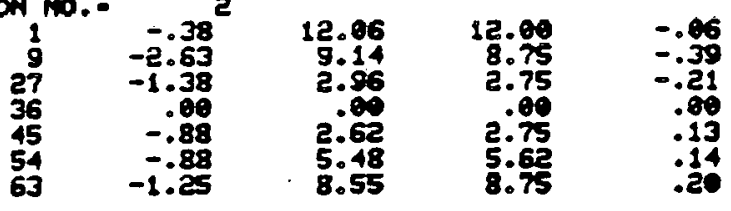

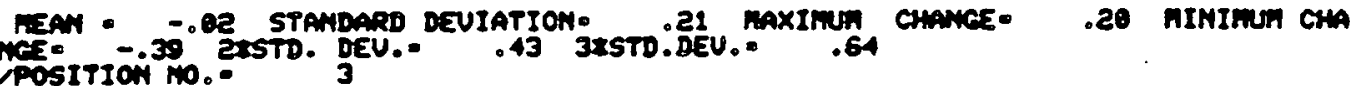

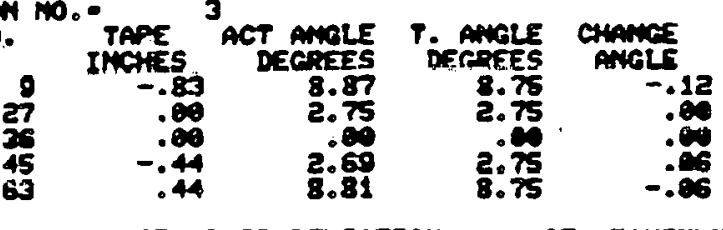
MEAM - -.12 


\begin{tabular}{|c|c|c|c|c|}
\hline $\begin{array}{l}\text { POSITION } \\
\text { SLAT NOT. }\end{array}$ & TAPE & ACT. ANGLE & T. AMGLE & CHAMCE \\
\hline $\begin{array}{l}3 \\
12 \\
15 \\
18 \\
21 \\
24 \\
27 \\
36 \\
39 \\
42 \\
45 \\
48 \\
51 \\
54 \\
57 \\
68 \\
63 \\
66 \\
69\end{array}$ & $\begin{array}{r}197.00 \\
71.62 \\
61.03 \\
51.75 \\
42.70 \\
34.25 \\
25.59 \\
7.09 \\
7.69 \\
16.12 \\
24.56 \\
33.38 \\
42.38 \\
51.47 \\
69.81 \\
79.62 \\
81.75 \\
99.75 \\
185.85\end{array}$ & $\begin{array}{r}11.65 \\
8.89 \\
6.84 \\
5.90 \\
4.92 \\
4.89 \\
3.86 \\
.80 \\
.71 \\
1.71 \\
2.65 \\
9.74 \\
4.80 \\
5.80 \\
6.77 \\
7.77 \\
9.81 \\
10.16 \\
11.38\end{array}$ & $\begin{array}{r}11.12 \\
7.66 \\
6.62 \\
5.62 \\
4.64 \\
3.69 \\
2.75 \\
.98 \\
.91 \\
1.83 \\
2.75 \\
9.60 \\
4.64 \\
5.62 \\
6.62 \\
7.66 \\
8.75 \\
9.89 \\
11.12\end{array}$ & $\begin{array}{r}.53 \\
.43 \\
.22 \\
.28 \\
.28 \\
.40 \\
.31 \\
.06 \\
-.20 \\
-.12 \\
-.16 \\
.95 \\
.16 \\
.18 \\
.15 \\
.11 \\
.11 \\
.26 \\
.27 \\
.26\end{array}$ \\
\hline $\begin{array}{r}\text { POSITION } 2 \\
1 \\
3 \\
9 \\
12 \\
15 \\
15 \\
18 \\
21 \\
24 \\
27 \\
39 \\
33 \\
36 \\
39 \\
42 \\
15 \\
51 \\
54 \\
57 \\
69 \\
63 \\
69\end{array}$ & $\begin{array}{r}116.57 \\
165.48 \\
82.06 \\
71.25 \\
61.12 \\
51.69 \\
42.81 \\
34.25 \\
25.50 \\
17.12 \\
8.53 \\
.8 .09 \\
8.25 \\
15.87 \\
24.31 \\
41.69 \\
59.88 \\
69.56 \\
79.67 \\
81.50 \\
106.53\end{array}$ & $\begin{array}{r}12.55 \\
11.26 \\
9.11 \\
7.97 \\
6.88 \\
5.88 \\
4.97 \\
4.09 \\
3.86 \\
2.12 \\
1.86 \\
.80 \\
.94 \\
1.60 \\
2.58 \\
4.54 \\
5.58 \\
6.08 \\
7.78 \\
8.94 \\
11.53\end{array}$ & $\begin{array}{r}12.00 \\
11.12 \\
8.75 \\
7.65 \\
6.62 \\
5.62 \\
4.64 \\
3.69 \\
2.75 \\
1.83 \\
.91 \\
.80 \\
.91 \\
1.83 \\
1.75 \\
4.64 \\
5.62 \\
6.62 \\
7.66 \\
8.75 \\
11.12\end{array}$ & $\begin{array}{l}.55 \\
.14 \\
.36 \\
.31 \\
.25 \\
.26 \\
.33 \\
.40 \\
.31 \\
.29 \\
.15 \\
.80 \\
.03 \\
-.23 \\
-.17 \\
-.10 \\
-.04 \\
.086 \\
.12 \\
.19 \\
.41\end{array}$ \\
\hline $\begin{array}{r}\text { Position } 3 \\
9 \\
12 \\
15 \\
18 \\
28 \\
21 \\
27 \\
39 \\
33 \\
36 \\
39 \\
42 \\
51 \\
57 \\
69 \\
63 \\
66\end{array}$ & $\begin{array}{r}81.63 \\
71.44 \\
61.32 \\
52.12 \\
42.56 \\
25.90 \\
16.62 \\
8.75 \\
7.90 \\
7.53 \\
16.12 \\
41.44 \\
60.10 \\
79.38 \\
82.12 \\
93.94\end{array}$ & $\begin{array}{r}8.98 \\
8.93 \\
6.94 \\
6.03 \\
4: 87 \\
2.89 \\
1.91 \\
1.15 \\
: 60 \\
: 64 \\
1: 71 \\
4.44 \\
6.55 \\
7.69 \\
9.13 \\
10.35\end{array}$ & $\begin{array}{r}8.75 \\
7.66 \\
6.62 \\
5.62 \\
4: 64 \\
2.75 \\
1.83 \\
: 91 \\
: 90 \\
1.81 \\
1.83 \\
4.64 \\
6.62 \\
7.65 \\
8.75 \\
9.89\end{array}$ & 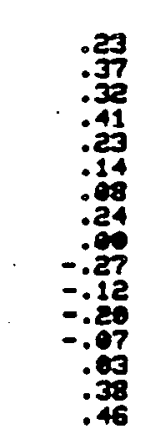 \\
\hline 69 & 105.69 & 11.31 & 11.12 & .19 \\
\hline
\end{tabular}




$\begin{array}{ll}\text { DATE : 4/8/80 } & \text { INSPECTION NO: } 12 \mathrm{a} \\ \text { CONCAVE } & \text { FURM/PANEL NO: }\end{array}$

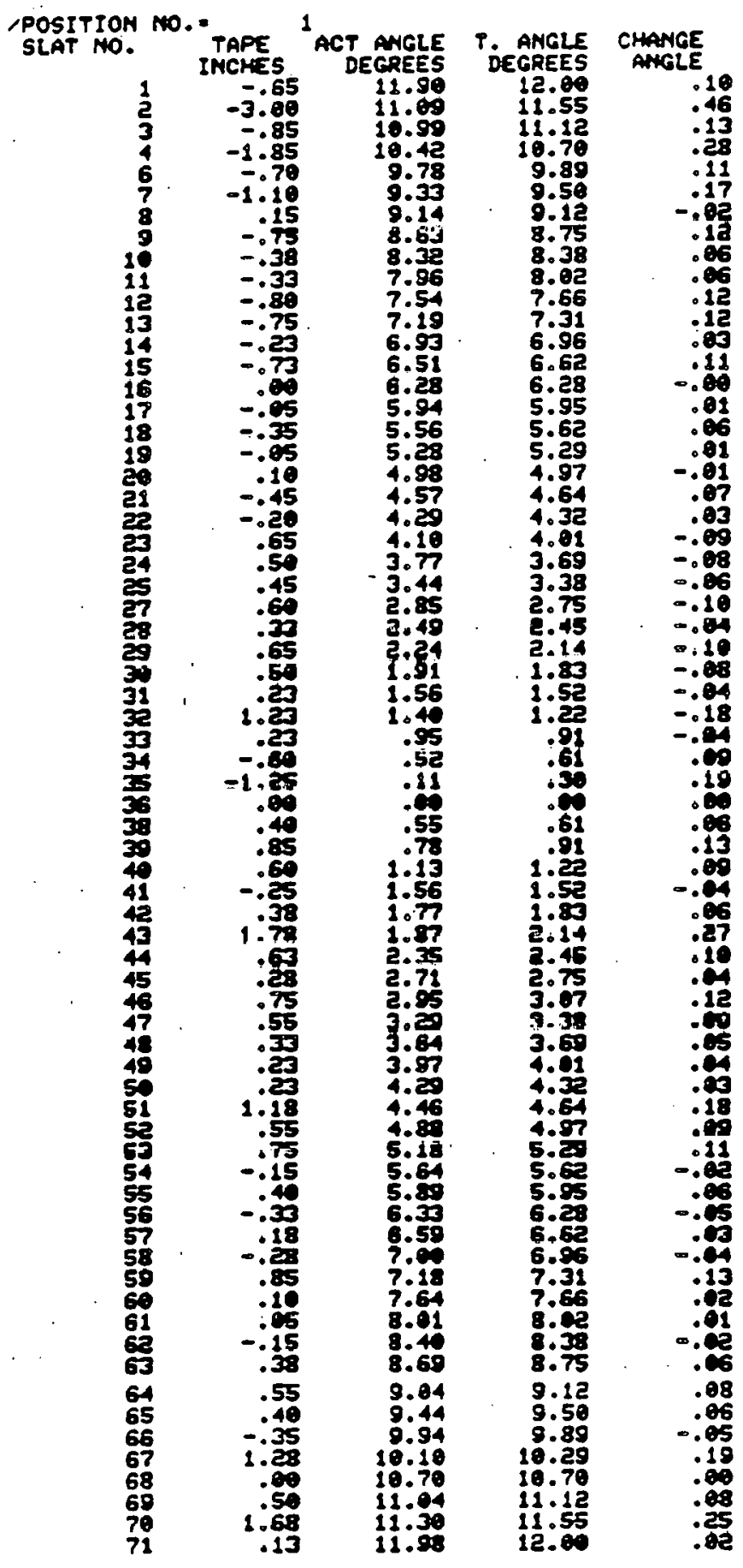




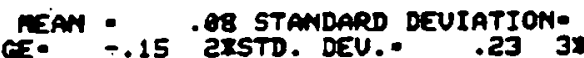

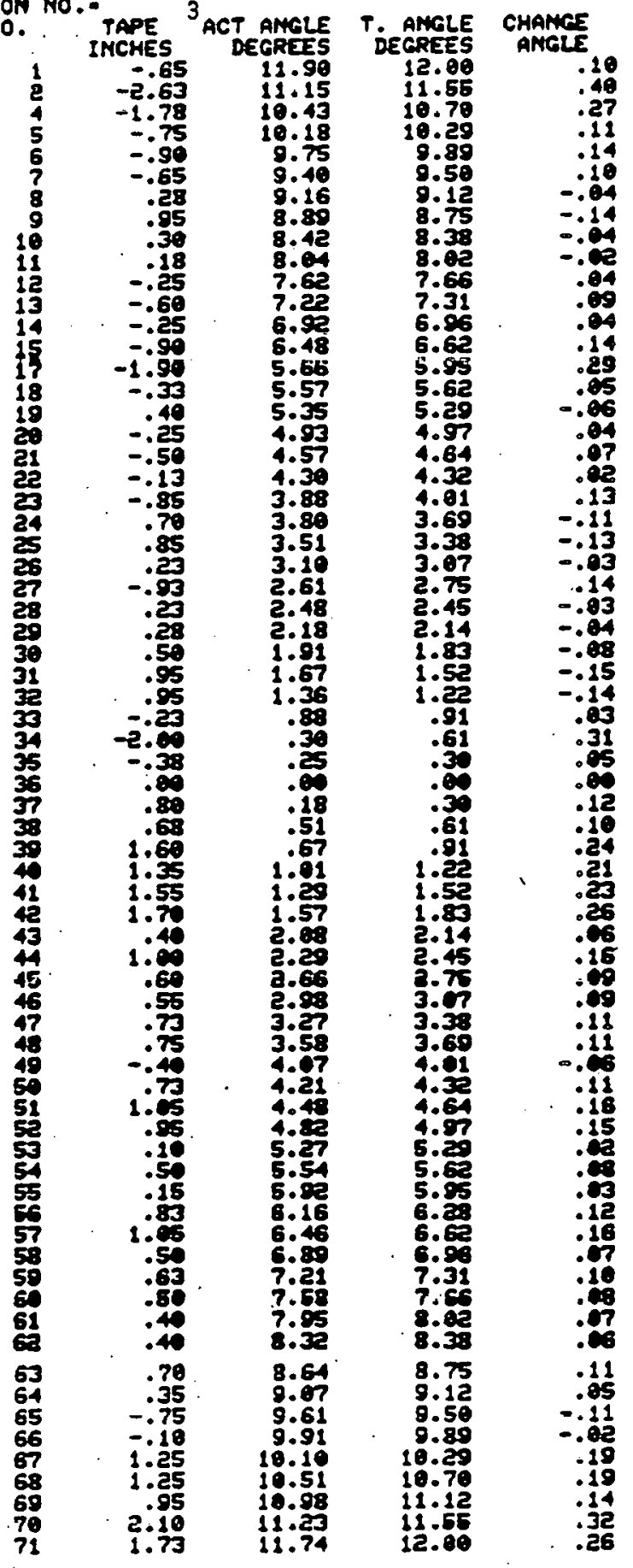

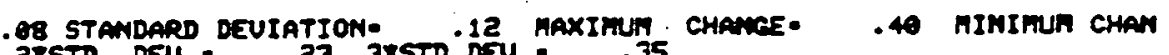




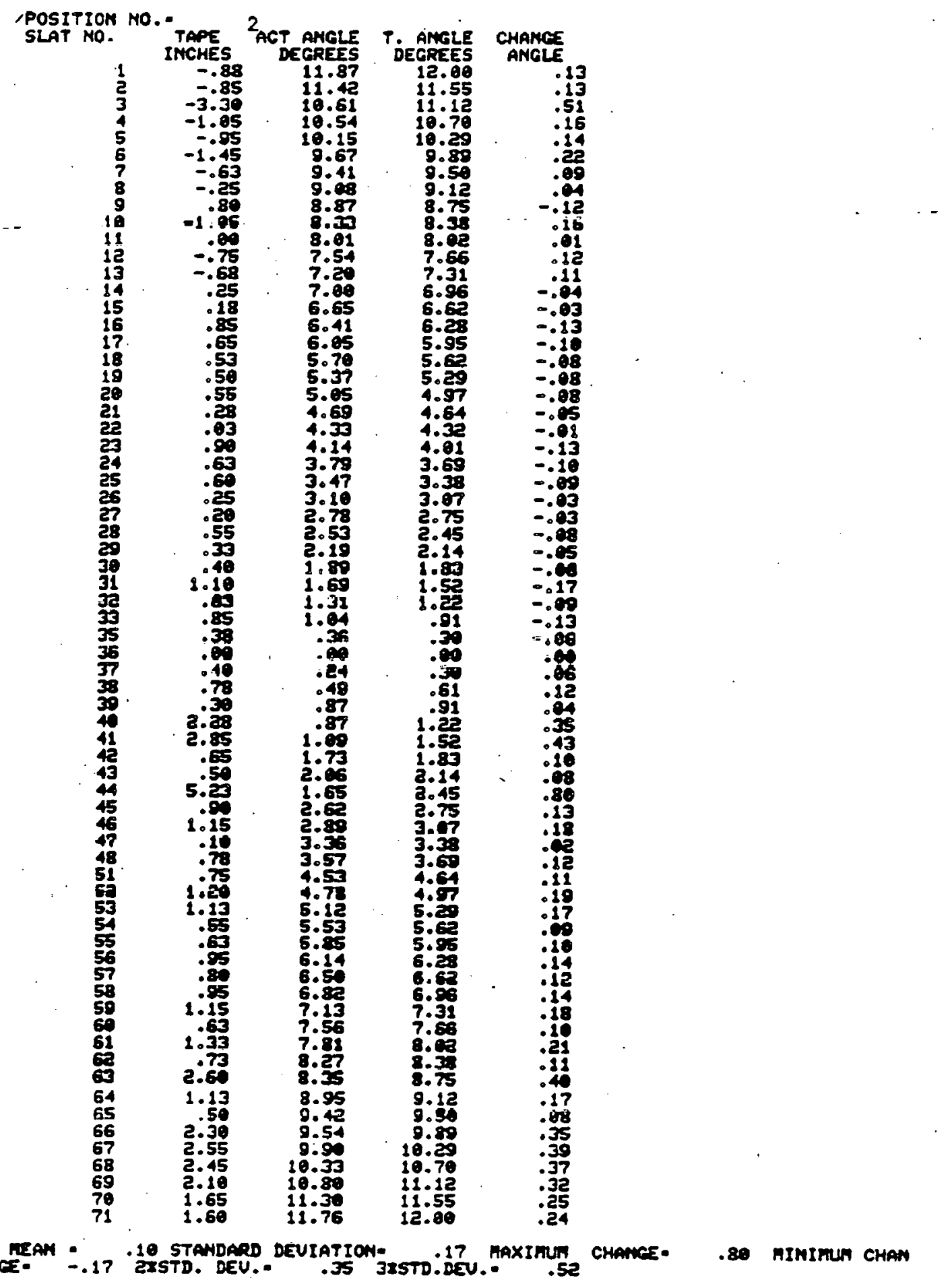




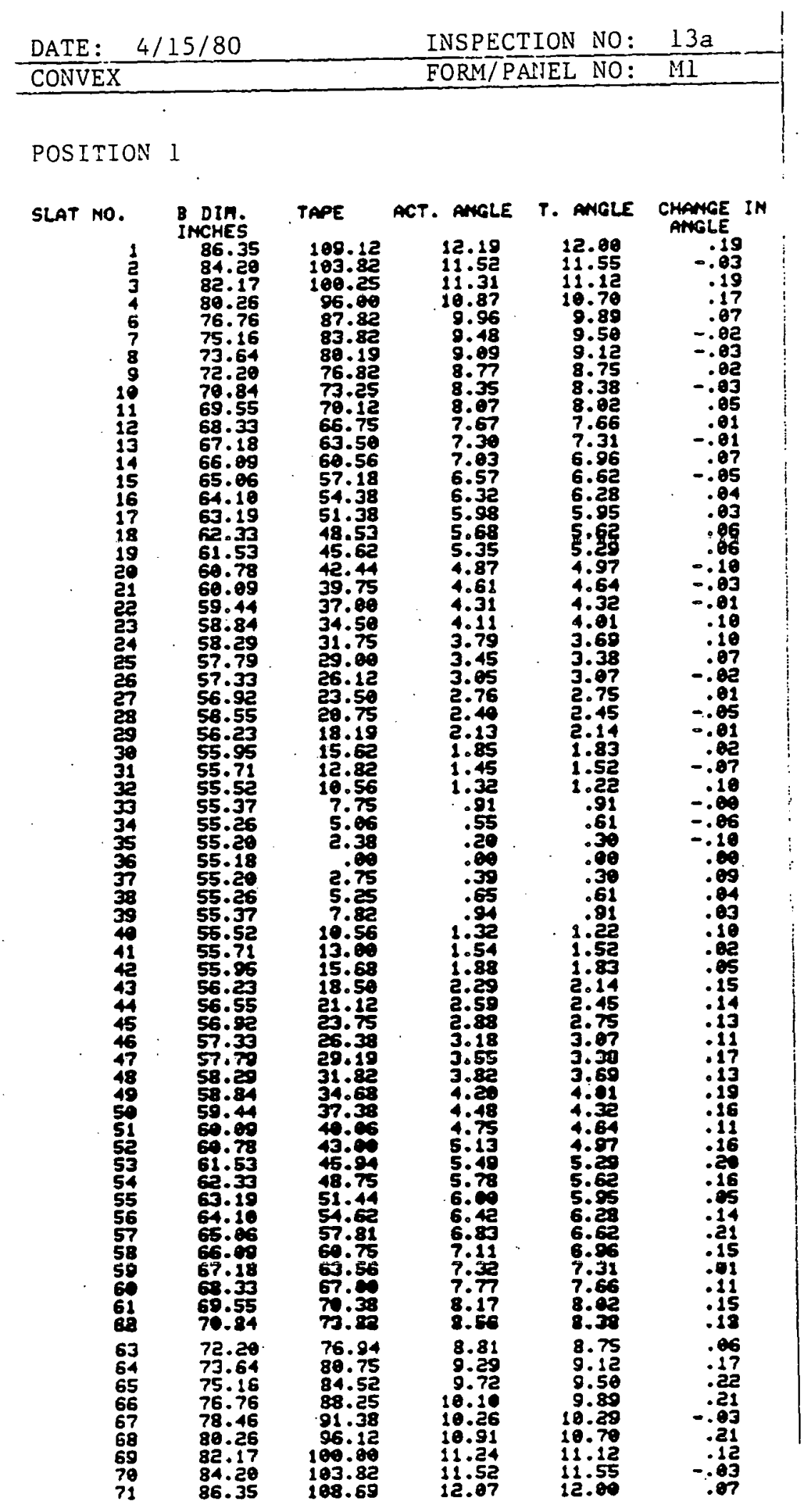




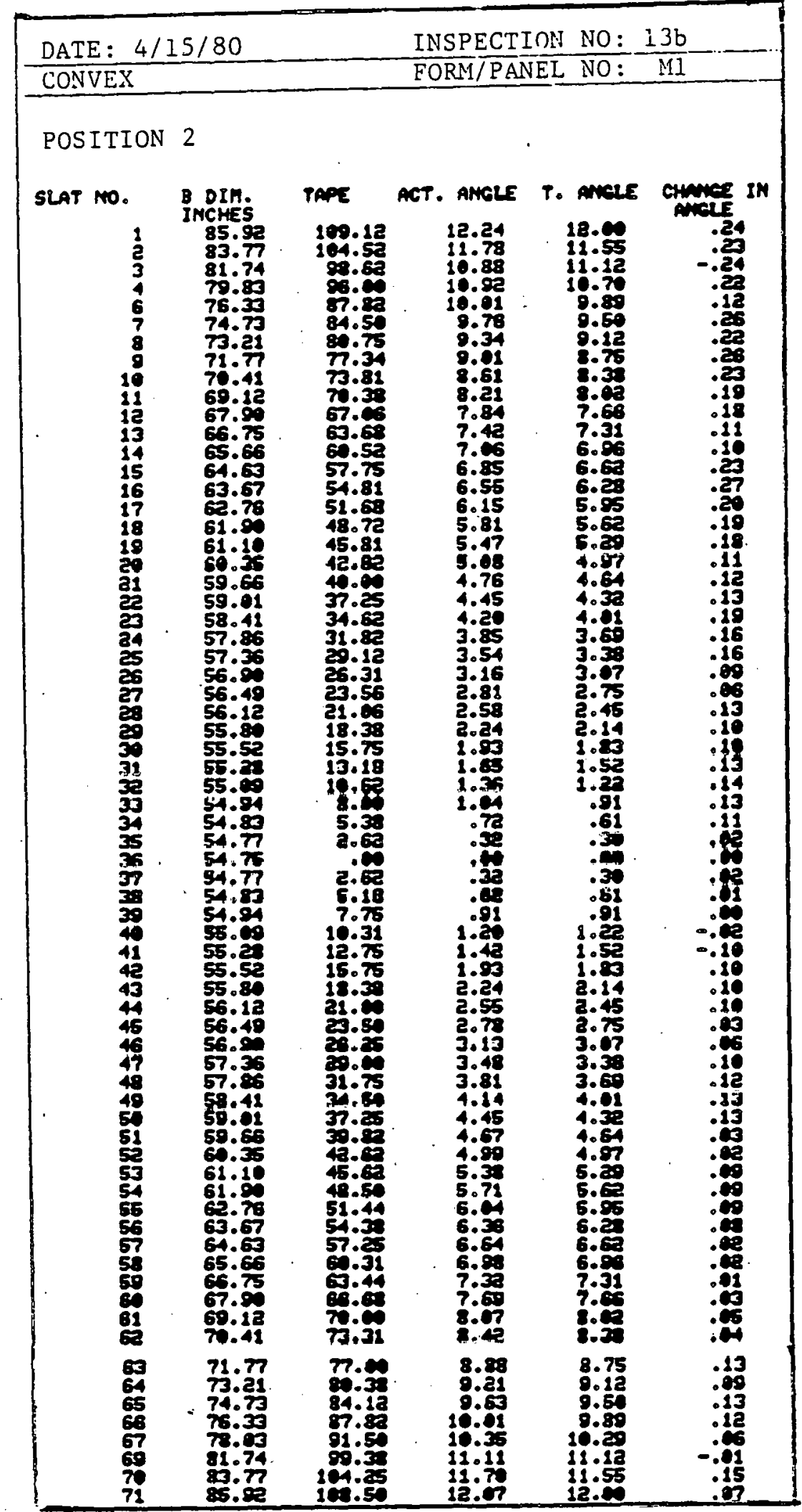




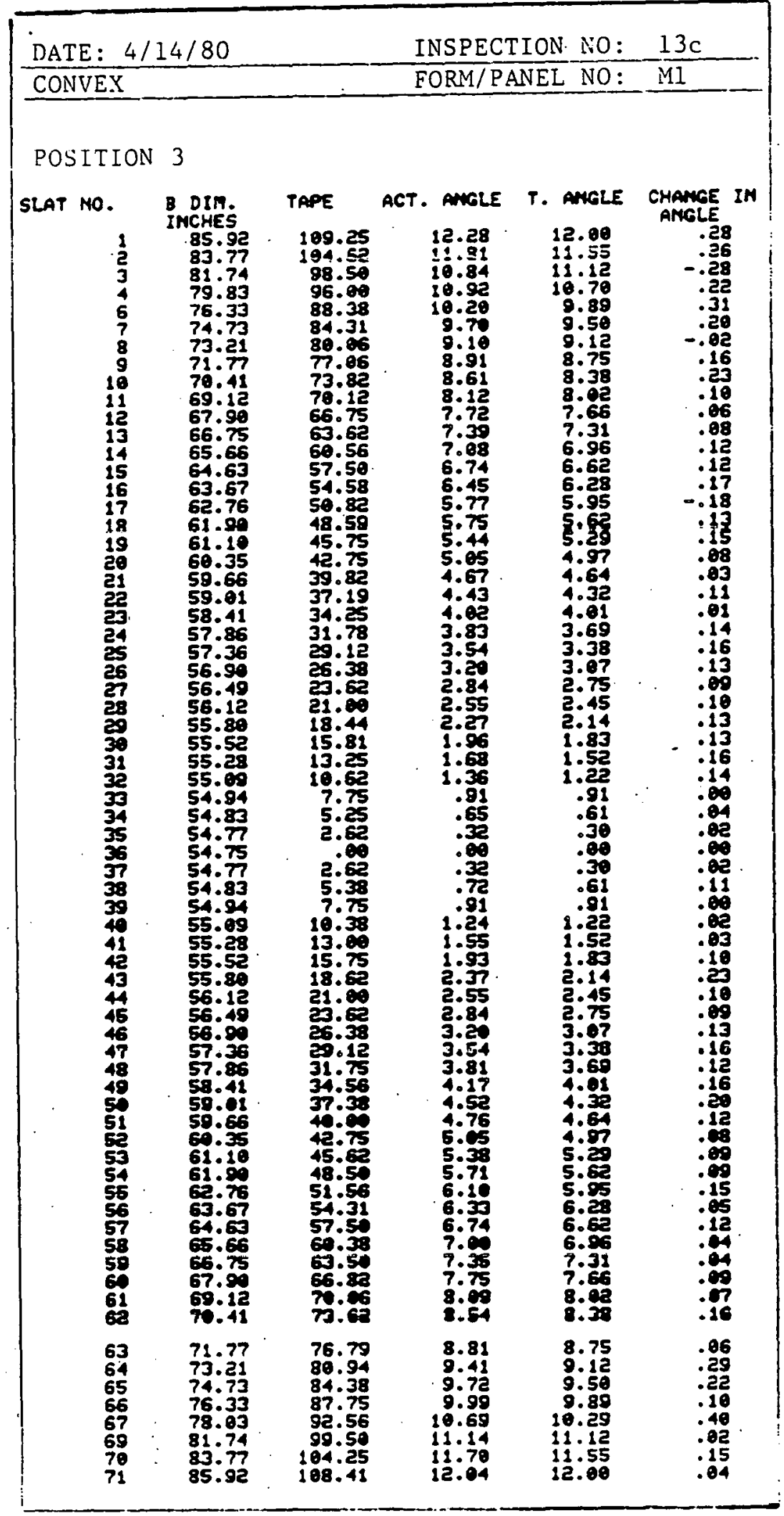


APPENDIX 3

COMPARISON OF SLAT ANGLES OF PANELS AND FORMS

\begin{tabular}{|c|c|}
\hline Difference Between & $\begin{array}{c}\text { Difference Betveen } \\
\text { Inspection } \\
\text { No. }\end{array}$ \\
\hline Initial and final $M 1$ & 1 and 13 \\
\hline Initial and final M2 & 2 and 12 \\
\hline Tnitial M1 and inititial M2 & 1 and 2 \\
\hline Final $M 1$ and final $M 2$ & 13 and 12 \\
\hline Final $M I$ and initial PMI-1 & 13 and 4 \\
\hline Final M1 and inftial PM1-2 & 13 and 5 \\
\hline Final M3 and initial PM3-1. & 11 and 6 \\
\hline Final $M 3$ and final $M 2$ & 11 and 12 \\
\hline Initial M2 and initial Pill-1 & 2 and 4 \\
\hline Initial $\mathbb{1} 2$ and initial PMl-2 & 2 and 5 \\
\hline Initial PMI-1 and initial PM1-2 & 4 and 5 \\
\hline Initial PM1-1 and initial PM3-I & 4 and 6 \\
\hline Initial PM1-2 and initial PM3-1 & 5 and 6 \\
\hline
\end{tabular}


ANGLE COMPARISON .

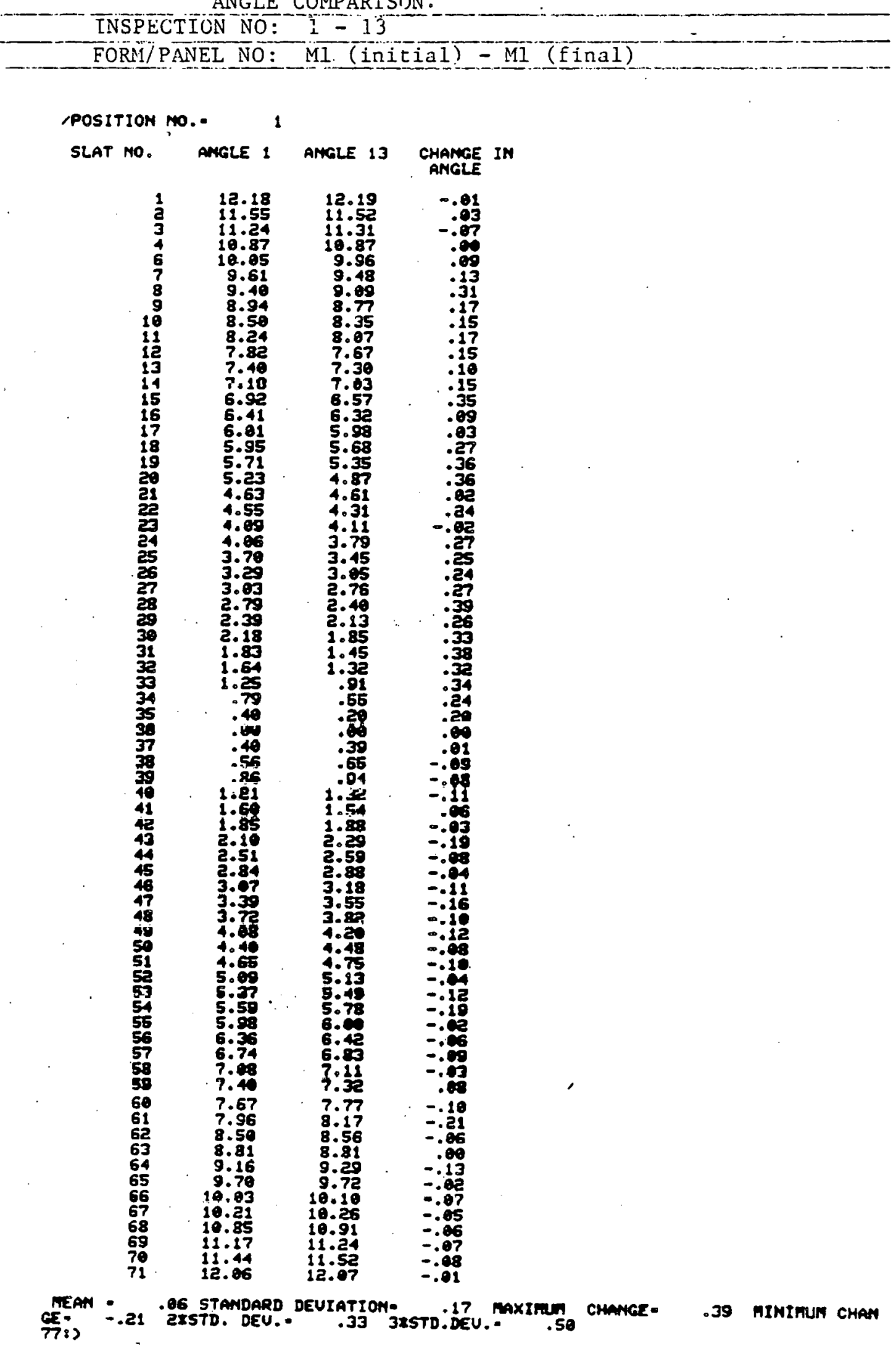


ANGLE COMPARISON

INSPECTION NO: $1-13$
FORM/PANEL NO: ML (initial) $=$ M1 (final)

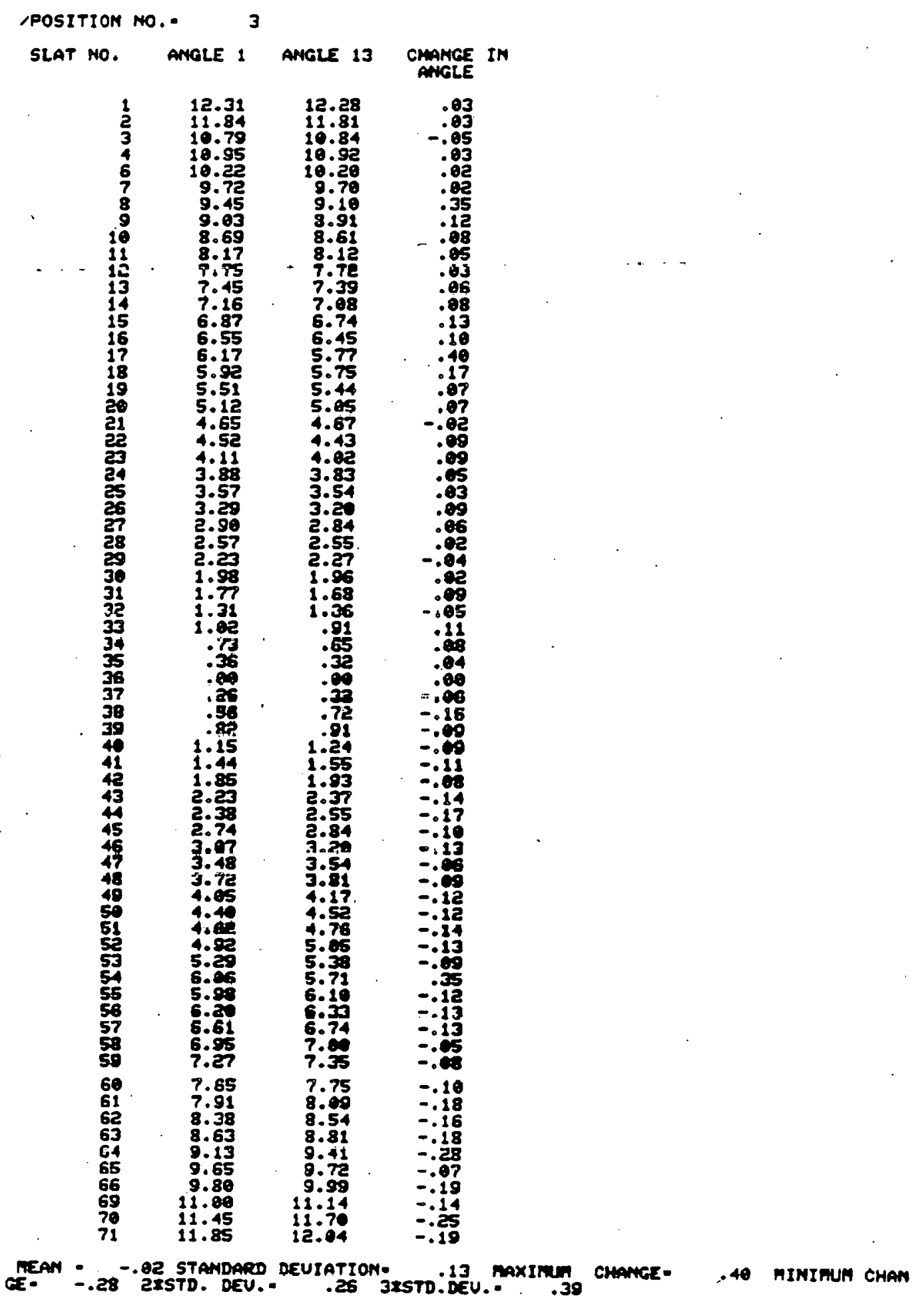


ANGLE COMPARISON

INSPECTION NO: $2-12$

FORM/PANEL NO: M2 (initial) - M2 (final)

position Mo.: 1

slat no. amgle 2 ancle 12 change IN

$\begin{array}{rrrr}1 & 11.88 & 11.80 & -.02 \\ 9 & 8.67 & 8.63 & .04 \\ 18 & 5.64 & 5.56 & -.08 \\ 27 & 2.83 & 2.85 & -.02 \\ 36 & .80 & .80 & .00 \\ 45 & 2.73 & 2.71 & .02 \\ 54 & 5.62 & 5.64 & -.08 \\ 63 & 8.72 & 8.69 & .83 \\ 71 & 11.99 & 11.98 & -.08\end{array}$

MEAN - .00 STAMDARD DEUIATION-

.04 MAXINUM CHANCE. .68 MINIRUA CHAM PPOSITION NO.:-

slat mo. ancle 2 angle 12 change in

$\begin{array}{rrrr}1 & 11.86 & 11.87 & -.01 \\ 9 & 8.76 & 8.87 & -.11 \\ 18 & 5.67 & 5.70 & -.03 \\ 27 & 2.93 & 2.78 & .15 \\ 36 & .80 & .80 & -.00 \\ 45 & 2.60 & 2.62 & -.02 \\ 54 & 5.60 & 5.53 & .07 \\ 63 & 8.70 & 8.35 & -.35 \\ 71 & 11.52 & 11.76 & -.24\end{array}$

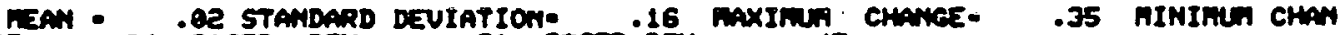

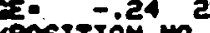

set mo

AMGLE 12

CHamas IN

$\begin{array}{rr}1 & 11.89 \\ 9 & 8.70 \\ 18 & 5.67 \\ 27 & 2.89 \\ 36 & .89 \\ 45 & 2.69 \\ 54 & 5.82 \\ 63 & 8.88 \\ 71 & 11.74\end{array}$

11.90
8.89
5.57
2.61
2.80
5.66
8.54
11.74

$-.01$

$-.19$

.10

.28

.03

.03

.24

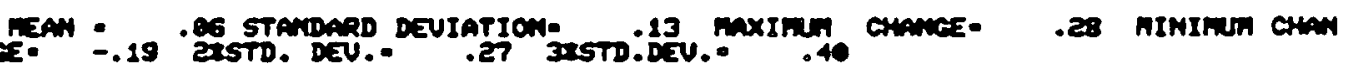
INT OCHATION ON ALL POSITIOUS

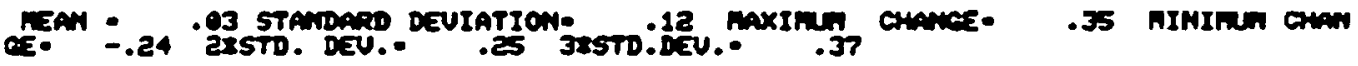


ANGLE COMPARISON

\begin{tabular}{l} 
INSPECTION NO: $\frac{1}{2}-2$ \\
\hline \\
FORM/PANEL NO:
\end{tabular}

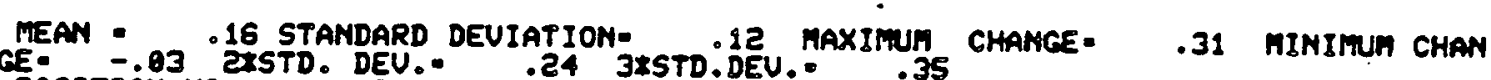
CPOSITION NO."

slat no. angle 1 angle 2 change in

$\begin{array}{rrrr}1 & 12.20 & 11.86 & .34 \\ 9 & 8.97 & 8.76 & .21 \\ 18 & 5.84 & 5.67 & .17 \\ 27 & 2.87 & 2.93 & -.06 \\ 36 & 0.00 & .00 & .00 \\ 45 & 2.68 & 2.60 & .08 \\ 54 & 6.09 & 5.60 & .40 \\ 63 & 8.78 & 8.79 & .98 \\ 11 & 12.03 & 11.52 & .51\end{array}$

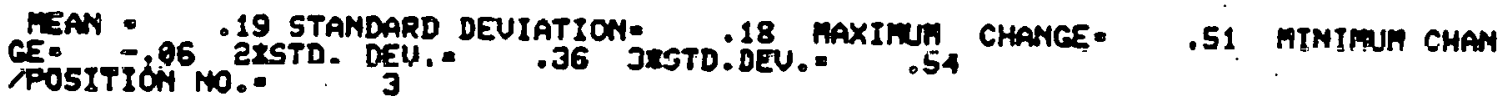

slat mo. amele 1 amgle 2 chamee in

$\begin{array}{rrrr}9 & 12.31 & 11.89 & .42 \\ 9 & 9.83 & 8.79 & .33 \\ 19 & 5.92 & 5.67 & .25 \\ 27 & 3.89 & 2.89 & .01 \\ 36 & .09 & .60 & .80 \\ 45 & 2.74 & 2.69 & .85 \\ 54 & 6.04 & 5.62 & .44 \\ 63 & 8.63 & 8.88 & -.25 \\ 71 & 11.85 & 11.74 & .11\end{array}$

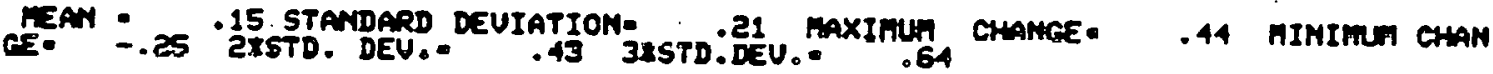
IMFORAATION ON ALL POSITIONS

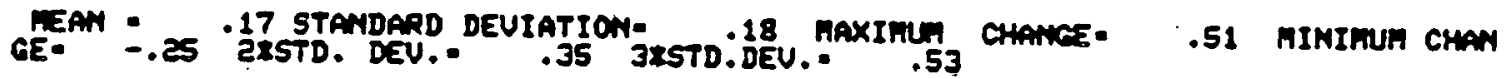


ANGLE COMPARISON

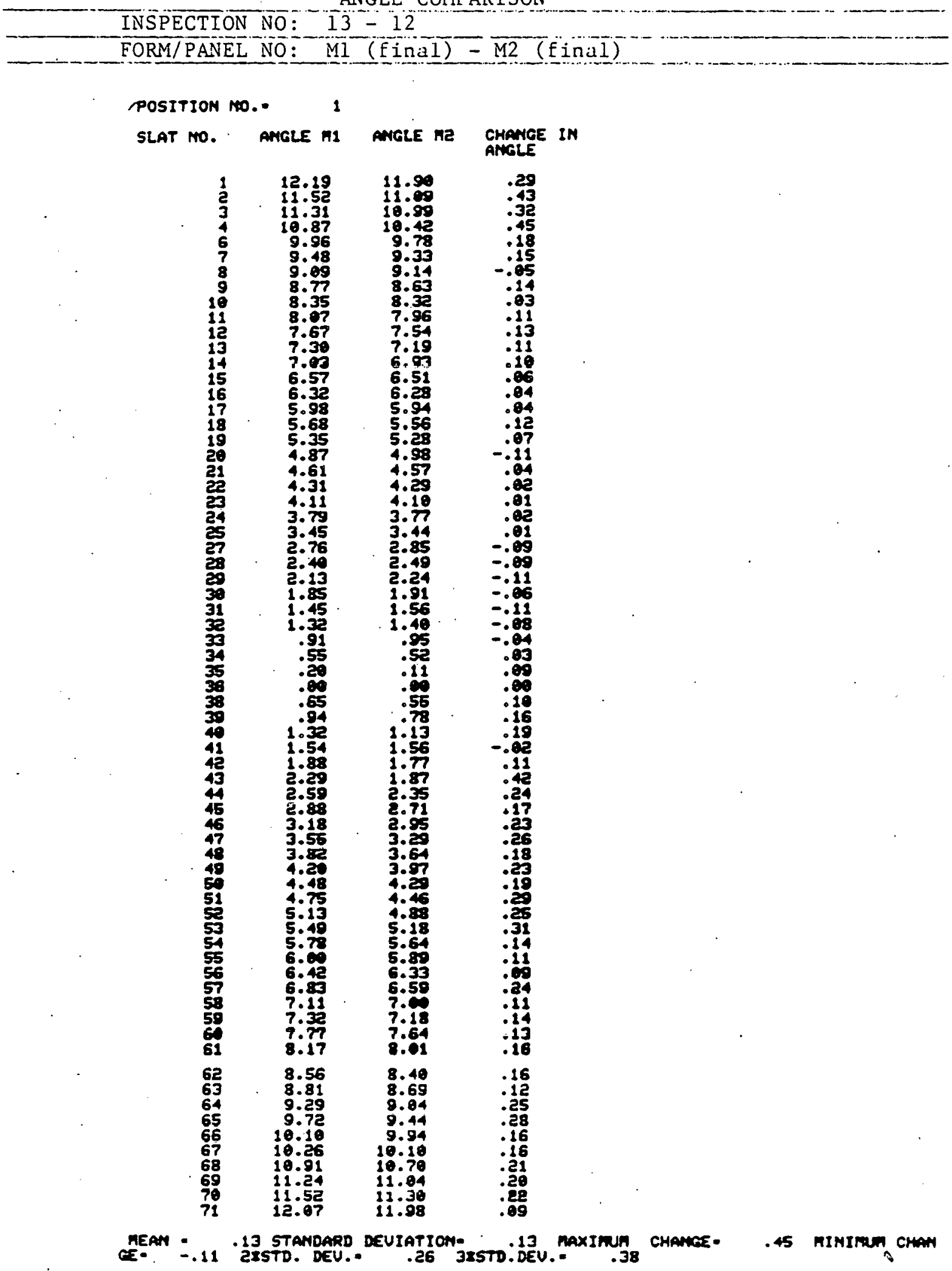


ANGLE COMPARISON

INSPECTION NO: 13 I2

FORM/PANEL NO: MI (final) - M2 (final)

IPOSITION NO.- $\quad$ ?

slat mo. aMGLE mI amole ne chance in

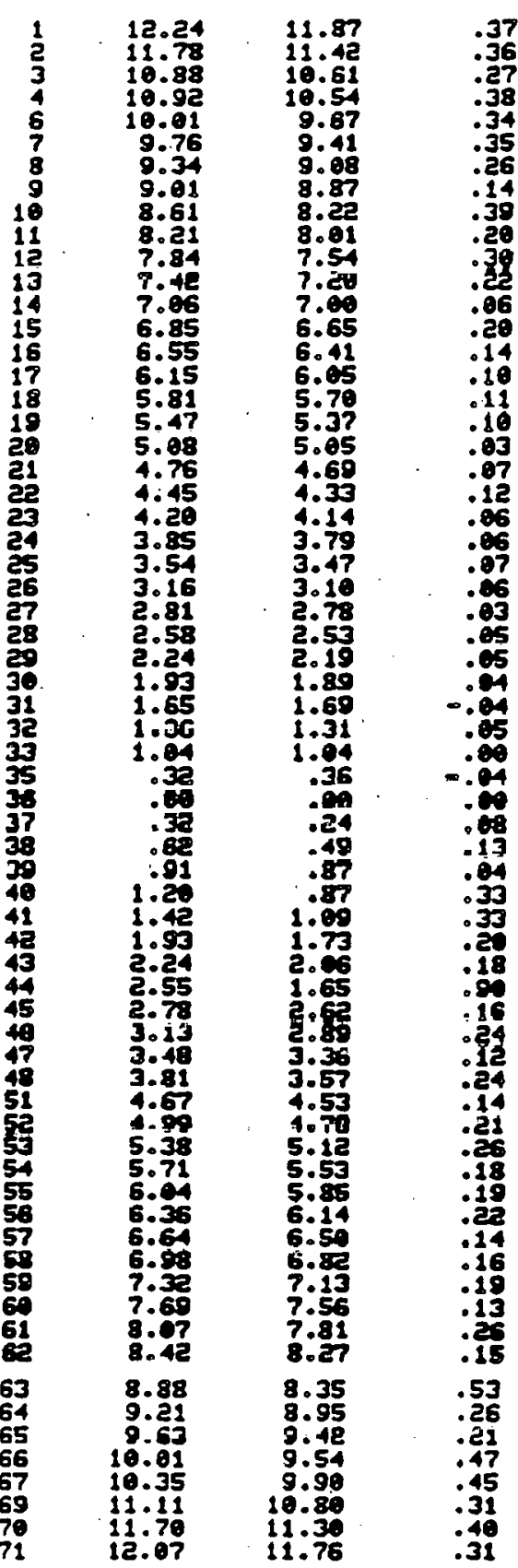

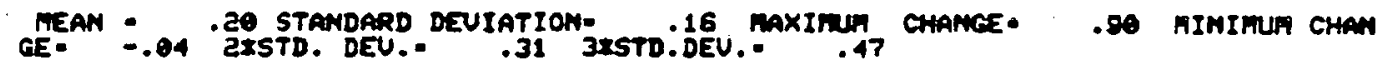




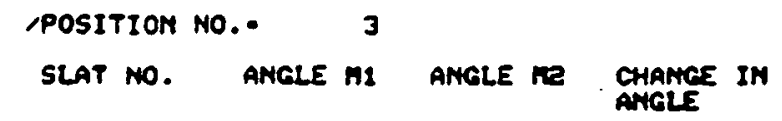

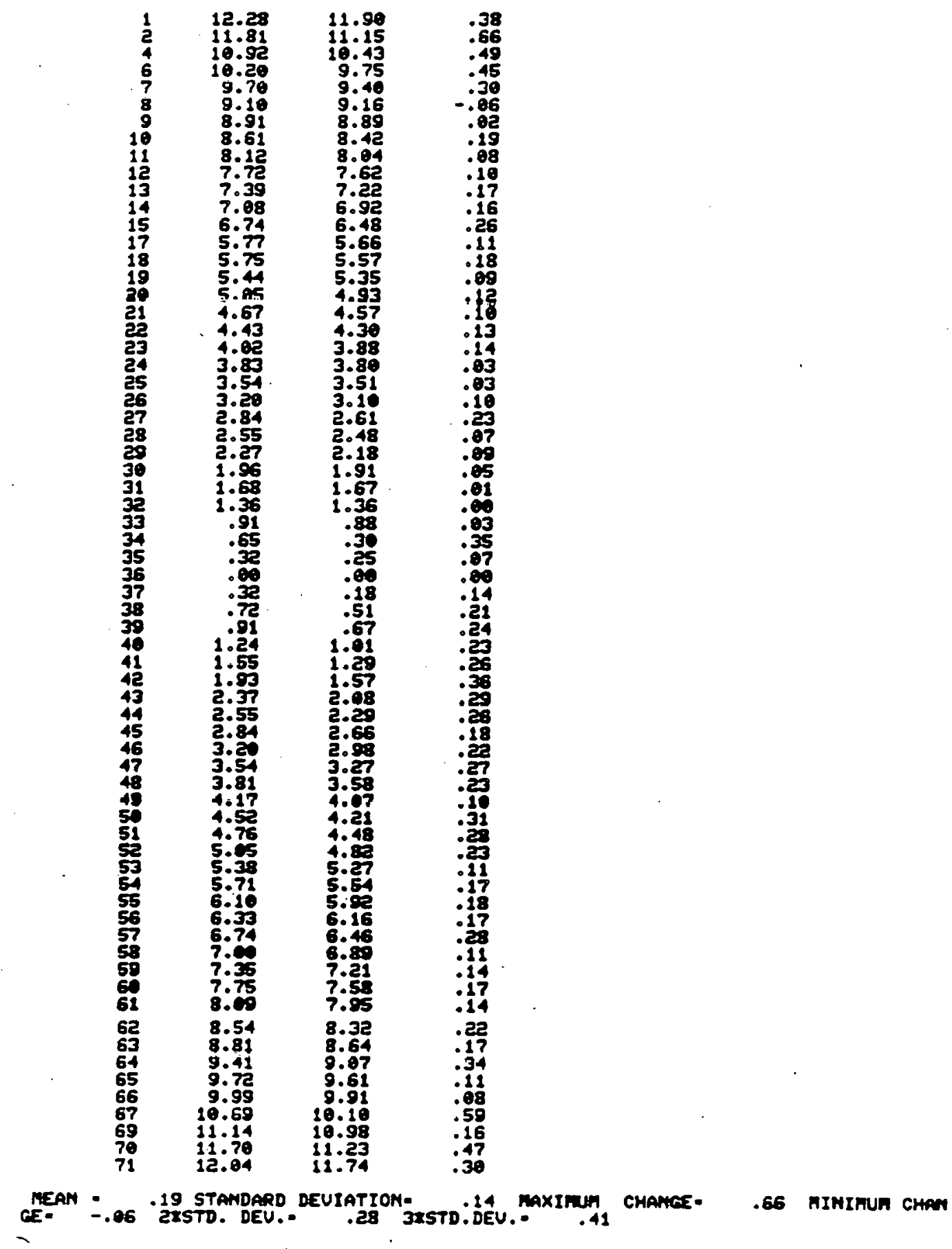


ANGLE COMPARISON

INSPECTION NO: $13=4$

FORM/PANEL NO: MI - PMI-1

IPOSITION NO.:

SLAT No. AMGLE NI AMGLE PNI-1. CHAMGE IN

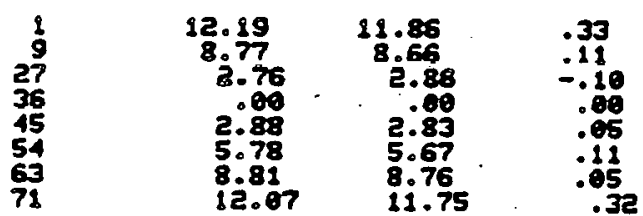

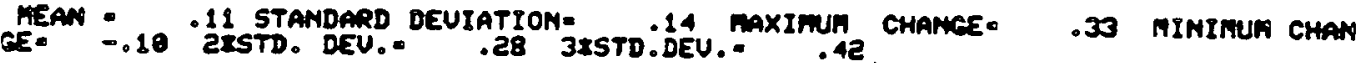


ANGLE COMPARISON

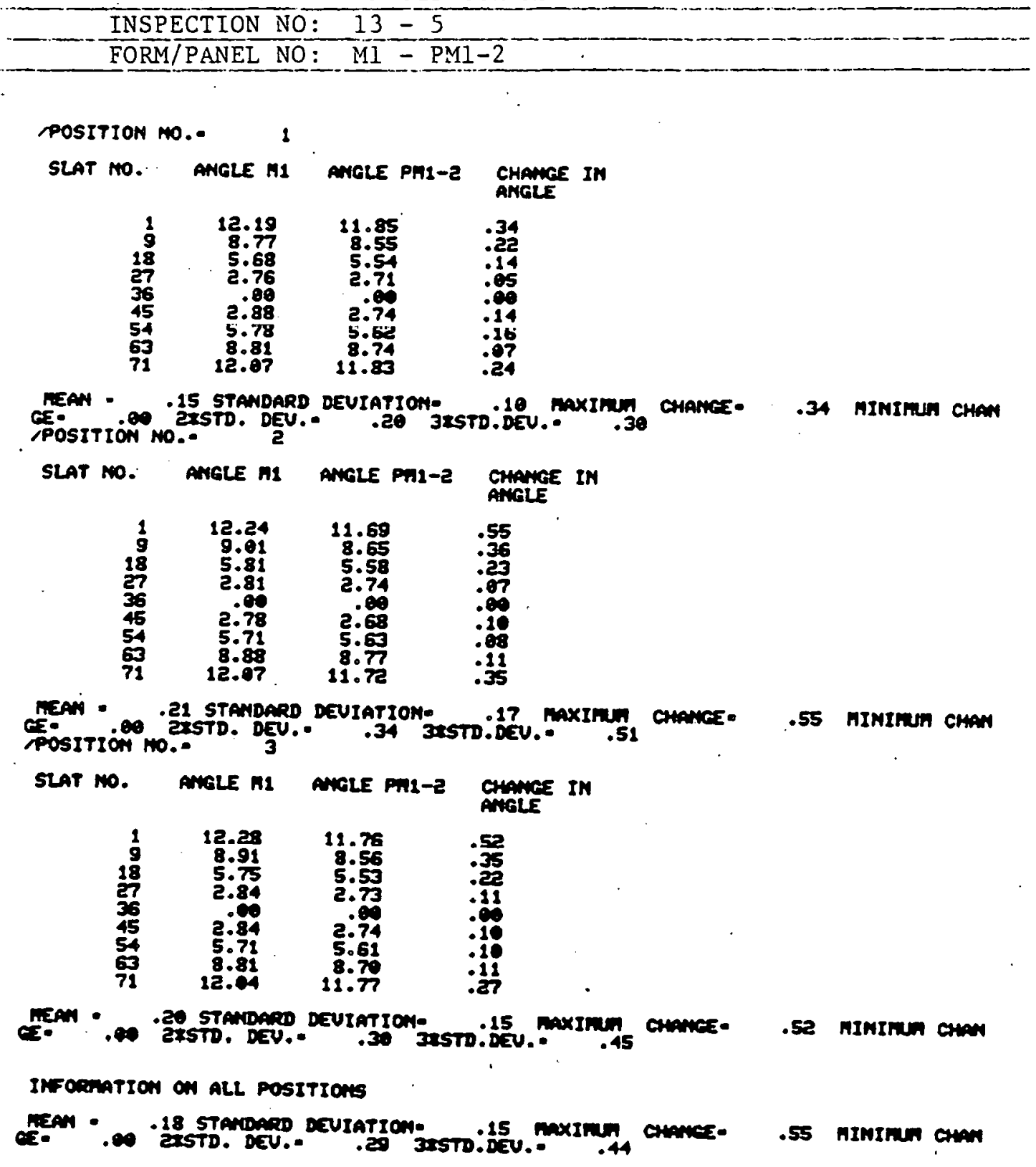


ANGLE COMPARISON

INSPECTION NO: $11-6$

FORM/PANEL NO: M3 - PM3-1

iposition no.e 1

slat no. amGle h3 amgle pho-1 chames IM

$\begin{array}{llll}18 & 5.90 & 5.63 & .27 \\ 27 & 3.06 & 2.95 & .11 \\ 36 & .00 & .09 & .09 \\ 45 & 2.65 & 2.71 & -.06 \\ 54 & 5.80 & 5.47 & .33 \\ 63 & 9.01 & 8.80 & .32\end{array}$

POSITION No.: 2

$\begin{array}{rrrr}1 & 12.55 & 12.86 & -.49 \\ 9 & 9.11 & 9.14 & -.03 \\ 18 & 5.88 & 6.03 & -.15 \\ 27 & 3.05 & 2.96 & .10 \\ 36 & .09 & .09 & .00 \\ 45 & 2.58 & 2.62 & -.04 \\ 54 & 5.58 & 5.18 & .10 \\ 63 & 8.94 & 8.55 & . .30\end{array}$

position no. 3

$\begin{array}{rrrr}9 & 8.98 & 8.87 & .11 \\ 18 & 6.83 & 6.07 & -.84 \\ 27 & 2.89 & 2.75 & .14 \\ 36 & 6.09 & 8.90 & .80 \\ 63 & 9.13 & 8.81 & .32\end{array}$

Ifrocanation ON ALL POSITIOHS

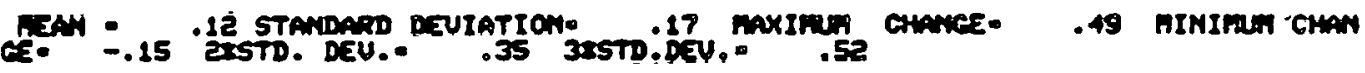


ALIGLE COMPARISON

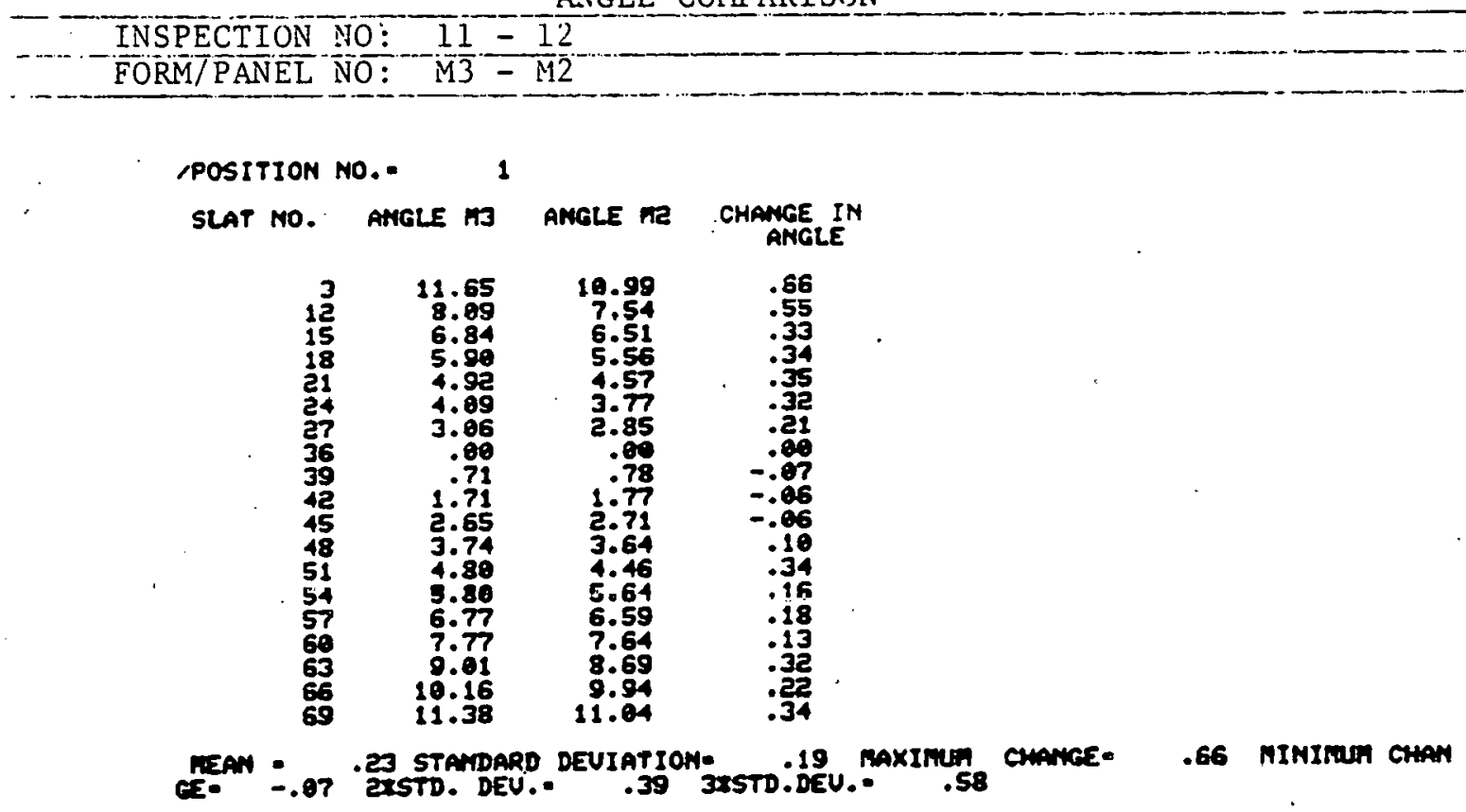

ANGLE COMPARISON

IINSPECTION NO: $11-12$

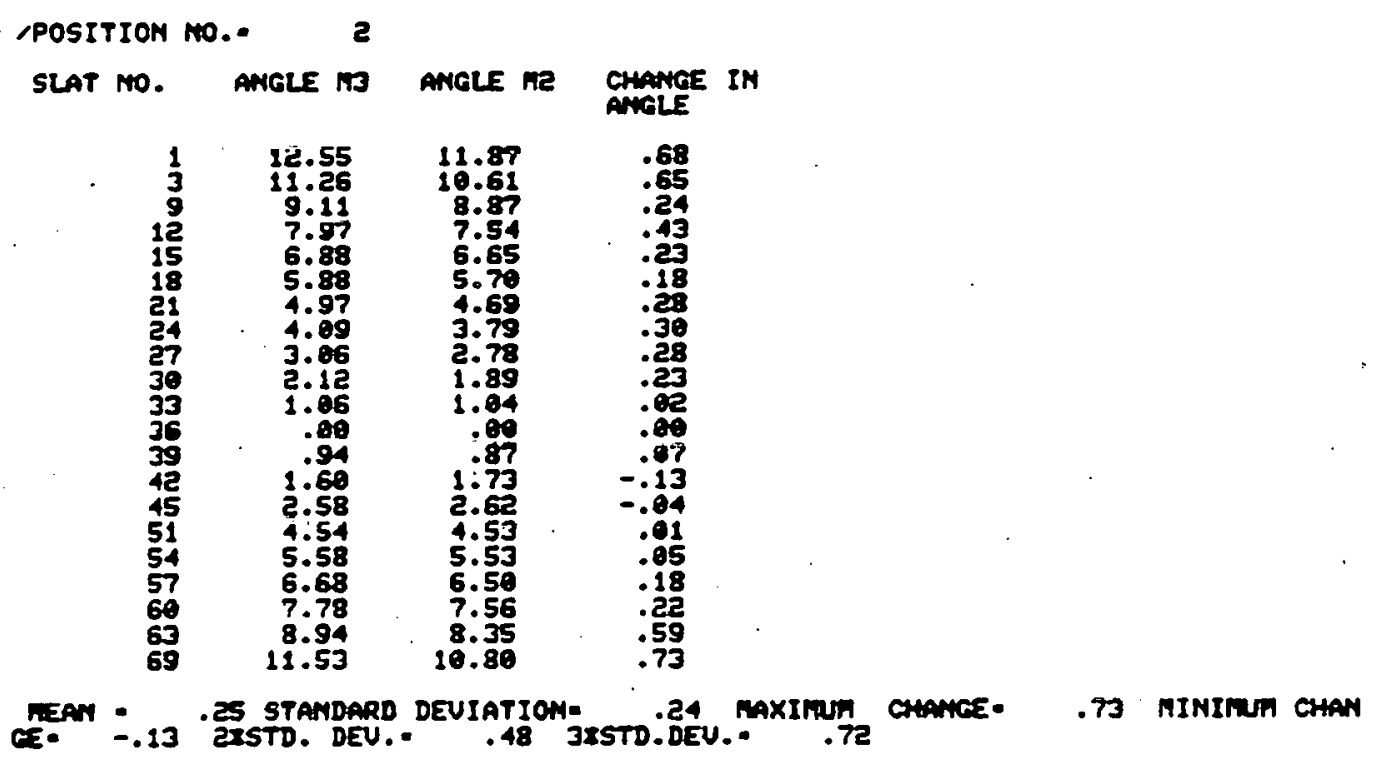




INSPECTION NO: $\frac{11-12}{\mathrm{M} 3}=\mathrm{M} 2$
FNRM/PANEL NO:

APOSITIOH HO.. 3

slat mo. amcle ho amele ne chames in

$\begin{array}{rrrr}9 & 8.98 & 8.89 & .09 \\ 12 & 8.93 & 7.62 & .41 \\ 15 & 6.94 & 6.48 & .46 \\ 18 & 6.03 & 5.57 & .46 \\ 28 & 4.87 & 4.57 & .38 \\ 27 & 2.89 & 2.61 & .28 \\ 30 & 1.91 & 1.98 & .09 \\ 33 & 1.15 & .88 & .27 \\ 36 & .60 & .80 & .08 \\ 39 & .64 & .67 & -.03 \\ 42 & 1.71 & 1.57 & .14 \\ 51 & 4.44 & 4.48 & -.04 \\ 57 & 6.55 & 6.46 & .09 \\ 60 & 7.69 & 7.58 & .11 \\ 63 & 9.13 & 8.64 & .49 \\ 66 & 19.35 & 9.91 & .44 \\ 69 & 11.31 & 18.98 & .33\end{array}$

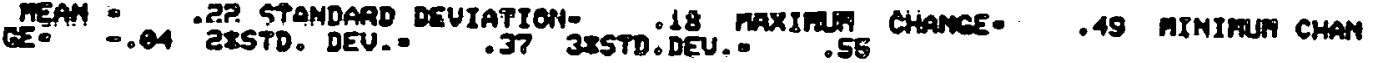


ANGLE COMPARISON

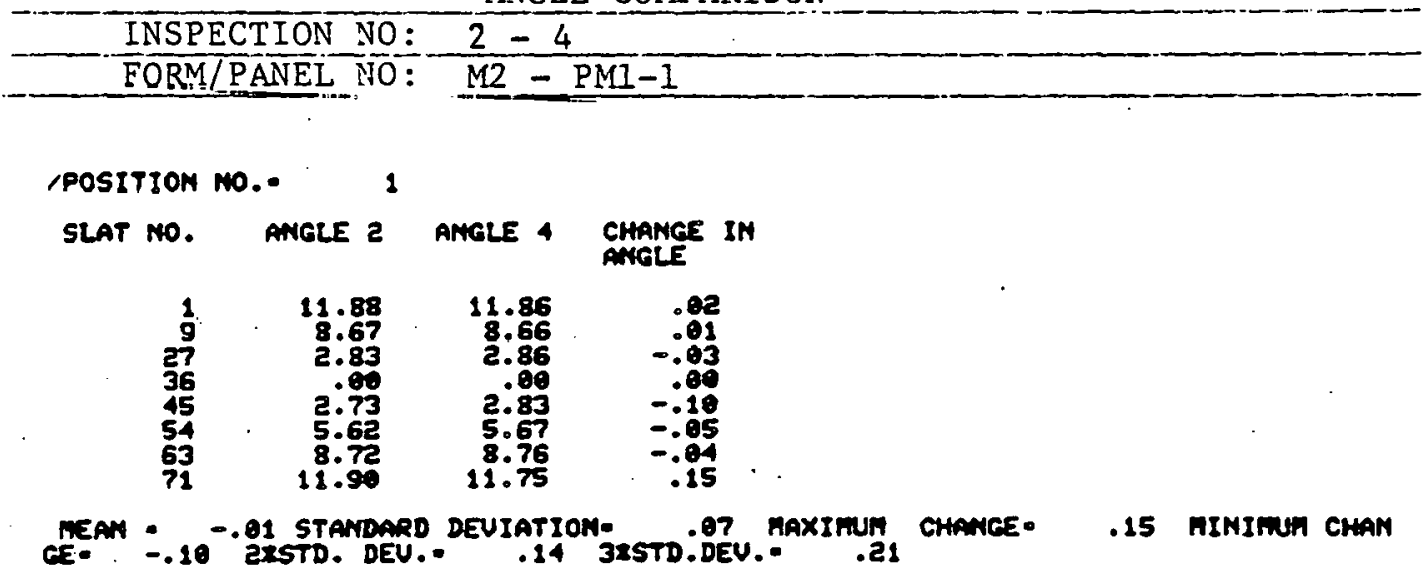


ANGLE COMPARISON

INSPECTION NO: $2-5$

FORM/PANEL NO: M2-PM1-2

POSITION MO.: 1

slat mo. amGle 2 angle 5 change in

$\begin{array}{rrrr}1 & 11.88 & 11.85 & .03 \\ 8 & 8.67 & 8.55 & .12 \\ 18 & 5.64 & 5.54 & .10 \\ 27 & 2.83 & 2.71 & .12 \\ 36 & .90 & .90 & .90 \\ 45 & 2.73 & 2.74 & -.91 \\ 54 & 5.62 & 5.62 & .69 \\ 63 & 8.72 & 8.74 & -.02 \\ 71 & 11.90 & 11.83 & .07\end{array}$

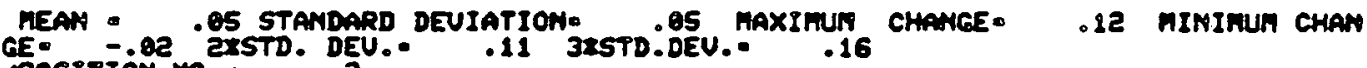
POSITION No."

slat mo. ancle 2 amgle 5 chamee in

$\begin{array}{rrrr}1 & 11.86 & 18.69 & .17 \\ 8 & 8.76 & 8.65 & .12 \\ 18 & 5.87 & 5.58 & .89 \\ 27 & 7.9 .7 & 2.74 & .10 \\ 38 & 2.60 & .06 & .09 \\ 45 & 2.60 & 2.68 & -.98 \\ 54 & 5.60 & 5.63 & -.63 \\ 63 & 8.79 & 8.77 & =.97 \\ 71 & 11.52 & 11.72 & -.20\end{array}$

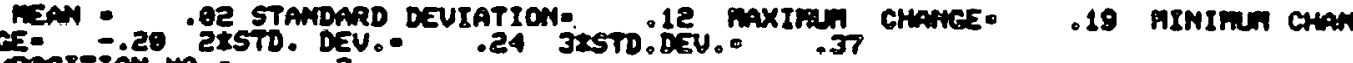
positson mo.:

SLAT mo. AMGLE 2 angle 5 change IM

$\begin{array}{rrrr}1 & 11.89 & 11.76 & .13 \\ 9 & 8.70 & 8.56 & .14 \\ 18 & 5.67 & 5.53 & .14 \\ 27 & 2.89 & 2.73 & .16 \\ 38 & 2.60 & .90 & .80 \\ 45 & 2.69 & 2.74 & -.65 \\ 54 & 5.69 & 5.61 & .91 \\ 63 & 8.89 & 8.70 & -.18 \\ 71 & 11.74 & 11.77 & -.03\end{array}$

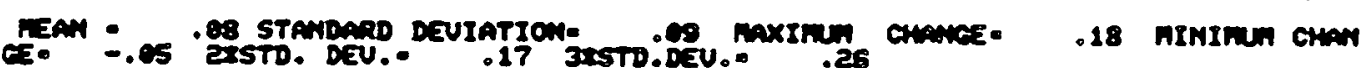
INF ORmation ON ALL POSITIONS

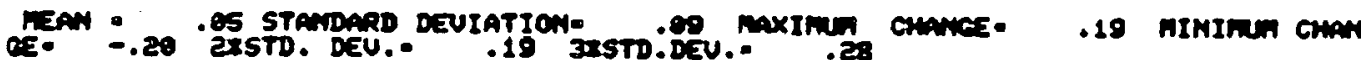


ANGLE COMPARISON

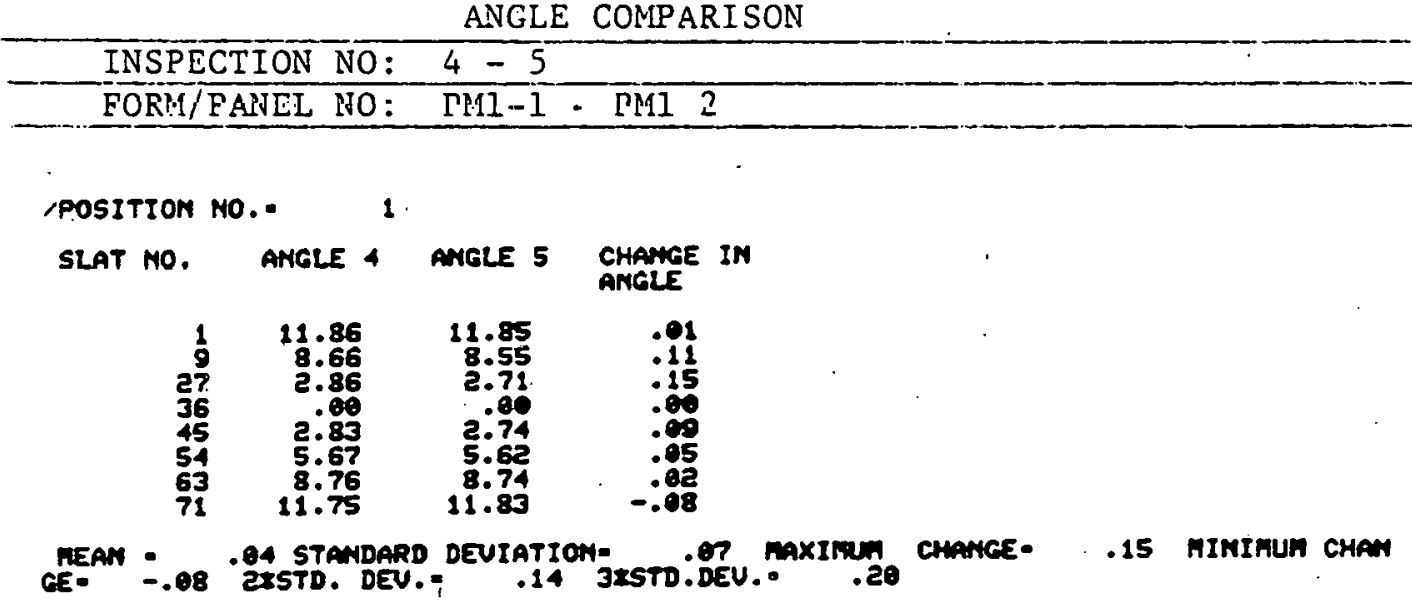


ANGLE COMPARISON

INSPECTION NO: $4-6$

FORM/PANEL NO: PMI-1 - PM3-1

MPOSITION MO.: 1

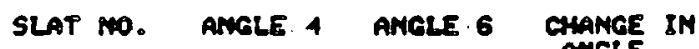

AMGLE

$\begin{array}{rrrr}9 & 8.66 & 8.77 & -.11 \\ 27 & 2.86 & 2.45 & -.89 \\ 36 & .80 & .08 & .00 \\ 45 & 2.83 & 2.71 & .12 \\ 54 & 5.67 & 5.47 & .20 \\ 63 & 8.76 & 8.60 & .07\end{array}$

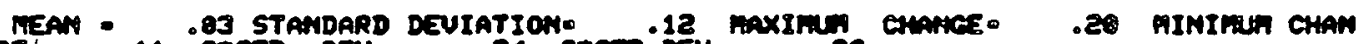


ANGLE COMPARISON

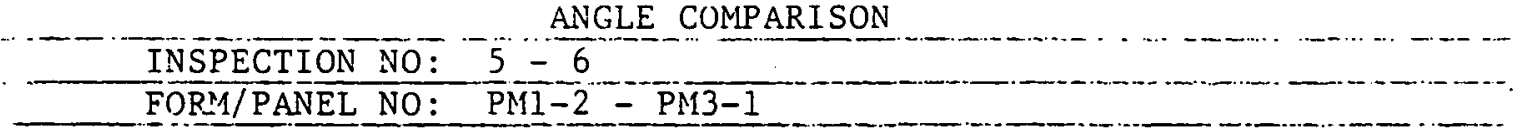

IPOSITION NO.- 1

slat no. amGle 5 amgle 6 change in

$\begin{array}{rrrr}9 & 8.55 & 8.77 & -.22 \\ 18 & 5.54 & 5.63 & -.09 \\ 27 & 2.71 & 2.95 & -.24 \\ 36 & .08 & .08 & .00 \\ 45 & 2.74 & 2.71 & .03 \\ 54 & 5.62 & 5.47 & .15 \\ 63 & 8.74 & 8.69 & .05\end{array}$

POSITION 2

slat no. amgle 5 amgle 6 chamee in

ANGLE

$\begin{array}{rrrr}1 & 11.69 & 12.06 & -.37 \\ 9 & 8.65 & 9.14 & -.49 \\ 18 & 5.58 & 6.03 & -.45 \\ 27 & 2.74 & 2.96 & -.22 \\ 36 & .06 & .00 & .06 \\ 45 & 2.68 & 2.62 & .06 \\ 54 & 5.63 & 5.48 & .15 \\ 63 & 8.77 & 8.55 & .22\end{array}$

POSITION 3

SLAT NO.

amGle 5 amole 6 chance IN

$\begin{array}{rrrr}9 & 8.56 & 8.87 & -.31 \\ 18 & 5.53 & 6.87 & -.54 \\ 27 & 2.73 & 2.75 & -.02 \\ 36 & 2.09 & .09 & .00 \\ 45 & 2.74 & 2.69 & .05 \\ 63 & 8.70 & 8.81 & -.11\end{array}$

INFORRATION ON ALL POSITIONS

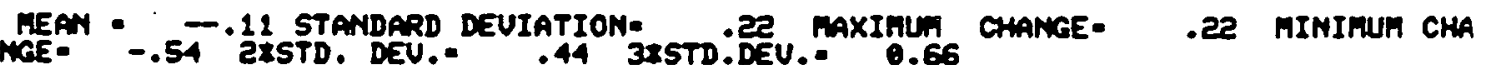




\section{(iㅛ.}

GENERAL ATOMIC

GENERAL ATOMIC COMPANY

P. O. BOX 81608

SAN DIEGO, CALIFORNIA 92138 